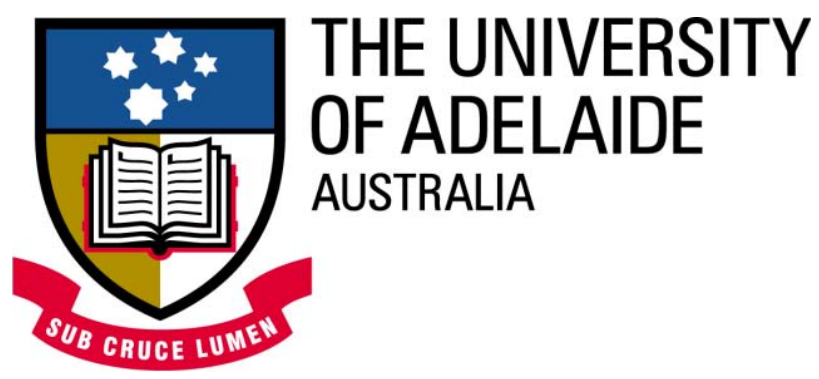

\title{
The working principle of a
} Ranque-Hilsch Vortex Tube

\author{
By \\ Yunpeng Xue
}

School of Mechanical Engineering

The University of Adelaide

South Australia 5005

Australia

A thesis submitted in fulfillment of the

Requirements for the degree of Ph.D. in

Engineering on the 21th of December 2012 


\section{Abstract}

A vortex tube is a Thermo-fluidic device, which generates cold and hot streams from a single injection of pressurized gas. Without any moving parts or chemical reaction within the tube, the interesting phenomenon of energy separation results only from fluid dynamic effects.

The main part of a typical counter-flow vortex tube is a straight tube with a tangential injection, through which compressed gas is injected into the tube. There are two exits, located at different ends of a counter-flow vortex tube, or at the same end for a uni-flow vortex tube. A control plug is positioned inside the tube away from the injection point, which has a smaller dimension than the inner diameter of the tube, and this allows the gas to escape from the small gap between the control plug and the tube. The cold exit is located in the central part of the tube at the same end of the injection, while the hot exit is the gap between the plug and the tube. When the compressed gas is injected into the tube tangentially at a high velocity, two streams with different temperatures will be generated and exhausted from the two exits of the tube. This phenomenon of temperature separation in a vortex tube is known as the Ranque effect.

Several explanations for the energy separation in a vortex tube have been proposed since its invention. However, due to the complex internal flow, the nature of the energy separation in the vortex tube, is still unclear. The proposed hypotheses can only be used to explain part of the phenomenon and they do not cover all the aspects of the temperature separation in the vortex tube. Therefore, to date, there has not been a well-accepted explanation for the thermal separation, and the flow behaviour inside the vortex tube remains unclear.

This thesis presents fundamental investigations on the Ranque-Hilsch Vortex Tube, with the aim to identify the dominant factors underlying in the energy separation phenomenon. This includes a critical review of current explanations, visualization of the flow pattern inside a water-operated vortex tube, accurate 
measurements of the flow properties inside an air-operated vortex tube, and a novel analysis based on the experimental data, which has led to a new understanding of the flow behaviour and the process of temperature separation.

Previous research on the vortex tube is summarized in the literature review and included theoretical, experimental and numerical investigations. The various explanations for the temperature separation have been examined and the different factors within these previous explanations were evaluated, and a new hypothesis was proposed.

Flow behaviour inside a vortex tube was visualized in this study. Visualization of the flow direction along a vortex tube indicated the existence of the main stream and oscillation of the vortex flow. For the first time, the flow behaviour inside a water-operated vortex tube was clearly visualized using different methods. It was observed that most of the cold flow came from the front part of the tube, specifically in the cold core. Also, the flow structure in the hot region was first visualized by dye and tracer particles. The visualization results agree well with the hypothesized flow behaviour and provide significant evidence in support of the concept proposed.

The proposed hypothesis is also supported by the results of the obtained velocity profiles. Obtained from the visualization results and measured in the airoperated vortex tube, the swirl velocity distributions near the hot end in both vortex tubes indicate the outwards flow and formation of a flow re-circulation region known as the multi-circulation. Calculations of the volumetric flow rate along the vortex tube indicate the existence and locations of the cold core and the multicirculation. The axial and radial velocity profiles in an air-operated vortex tube indicate the existence of the cold core. Formation of the multi-circulation is indicated by the 3-D velocity profiles in the rear part of the tube. Thus, the proposed hypothesis is strongly supported by the velocity profiles obtained in this study.

Exergy density analysis in the air-operated vortex tube was performed in this study and offered solid support for the proposed hypothesis. The analysis from this study, as well as the data from other studies, show a slightly decreased peripheral 
exergy density in the rear part of the tube, which defines the dominant contribution to the temperature rise from the stagnation and mixture due to multi-circulation. The dramatically reduced exergy density in the front part of the tube, together with an estimation of the temperature drop based on the forced vortex assumption, indicate that the pressure gradient in the front part of the tube is the primary factor contributing to the temperature drop.

Based on the proposed hypothesis, discussion of various geometrical effects, such as cold mass flow ratio, tube length, tube diameter and inlet nozzle, on the tube performance has been undertaken. A good agreement between the theoretical and experimental results demonstrates the validity of the proposed hypothesis for the temperature separation occurring within a vortex tube.

As a result of the study presented, a novel explanation for the temperature separation phenomenon inside a vortex tube can be forwarded, and can be supported by the flow visualization, measured flow velocity profiles and exergy analysis inside the vortex tube. The explanation is best described as a pressure gradient near the cold end being the main factor for temperature drop, while stagnation and fluid mixing due to the multi-circulation being the main reason for the temperature rise. 


\section{Declaration}

This work contains no material which has been accepted for the award of any other degree or diploma in any university or other tertiary institution and, to the best of my knowledge and belief, contains no material previously published or written by another person, except where due reference has been made in the text.

I give consent to this copy of my thesis when deposited in the University Library, being made available for loan and photocopying, subject to the provisions of the Copyright Act 1968.

The author acknowledges that copyright of published works contained within this thesis (as listed below) resides with the copyright holder(s) of those works.

I also give permission for the digital version of my thesis to be made available on the web, via the University's digital research repository, the Library catalogue and also through web search engines, unless permission has been granted by the University to restrict access for a period of time. 


\section{Acknowledgement}

I would like to acknowledge the efforts of all the people who have contributed towards the work in this thesis. I am deeply indebted to my principal supervisor, Dr. Maziar Arjomandi, without whom this thesis would have never been completed. Apart from appreciating his erudite suggestions on the research matters and his will to sharing the knowledge, I am extremely thankful to him for tolerating my unscheduled intrusions and great help in my personal life.

I would also like to express my honest gratitude to my co-supervisor, Assoc. Prof. Richard Kelso for his brilliant ideas in fluid dynamics and effective suggestions in the experiments. Special thanks to his detailed comments for the journal papers and obvious improvement of the publication quality.

I am also thankful to many other academics in our school who have helped me in my research, in particular the workshop staff. Thanks to Assoc. Prof. Ted McMurchie for his professional editing of the thesis.

I am grateful to my parent, for giving me their deep love, encouragement and support during my education. There are a lot of thanks I want to say to my wife, Minghui Sun, for her continuous encouragements and supports, not only in daily life but also in my research activities.

At the end, I want to dedicate this thesis to my little boy, Leo Xue. You are the best gift I have received during these years and your smile is the most effective magic to relieve all the tiredness and stress. 


\section{Table of content}

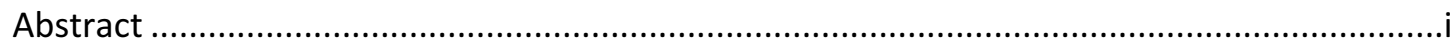

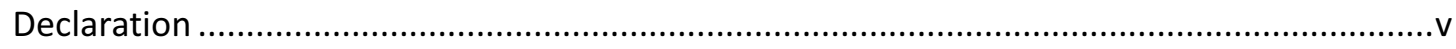

Acknowledgement .............................................................................................................

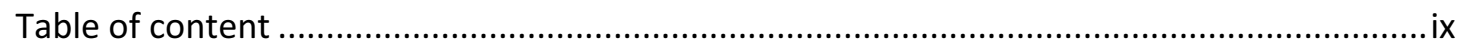

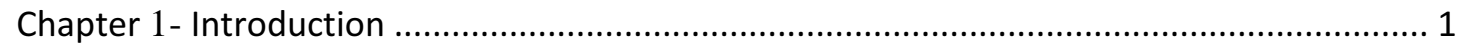

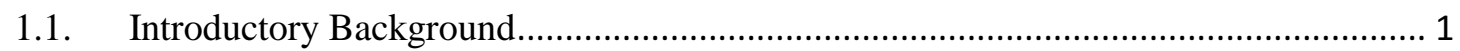

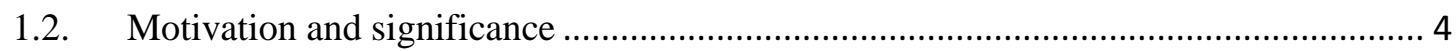

1.3. Publications arising from this thesis .................................................................... 5

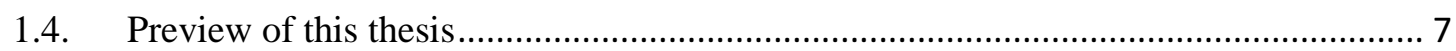

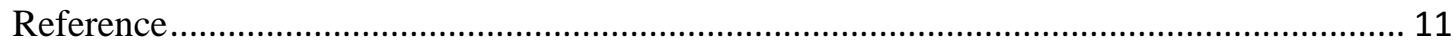

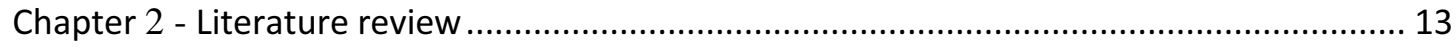

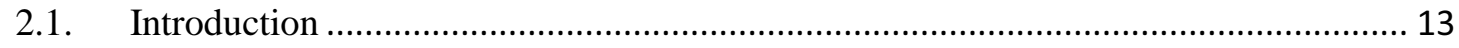

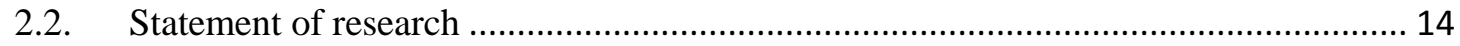

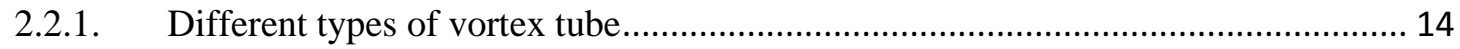

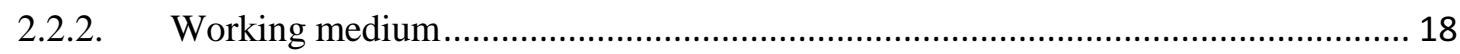

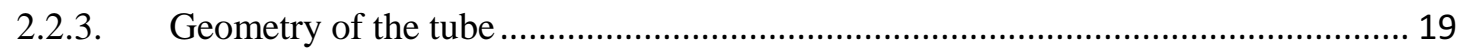

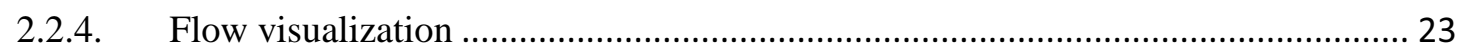

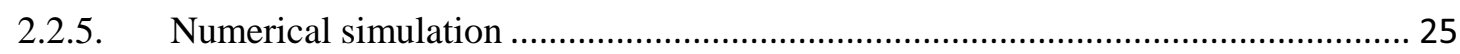

2.3. Research objectives and methodology ……............................................................. 29

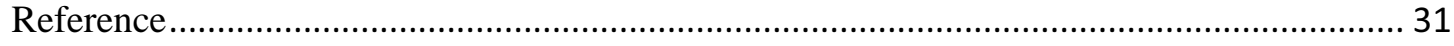

Chapter 3 - Analysis of the available hypothesises ................................................................. 39

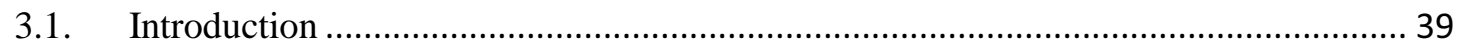

3.2. A critical review of temperature separation in a vortex tube ....................................... 40

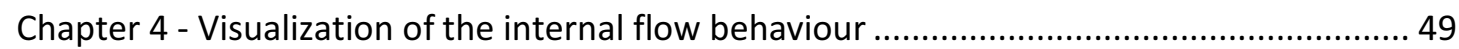

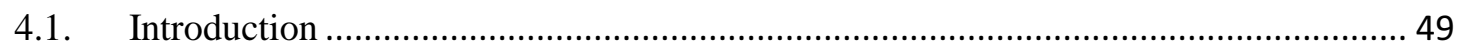

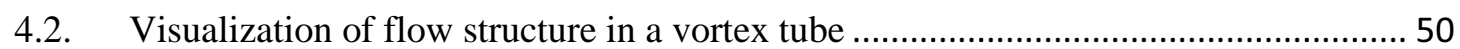

Chapter 5 - Flow behaviour inside an air-operated vortex tube........................................... 59

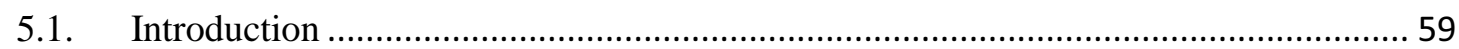

5.2. Experimental study of the flow structure in a counter flow vortex tube ......................6 60

5.3. Experimental study of the thermal separation in a vortex tube ....................................69

Chapter 6 - Analytical assessment of the proposed hypothesis ............................................ 79 


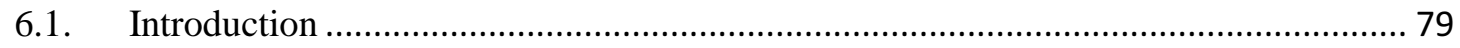

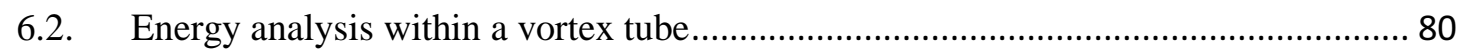

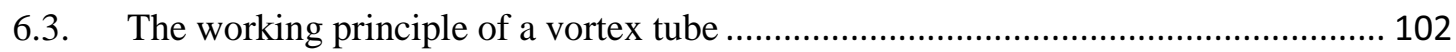

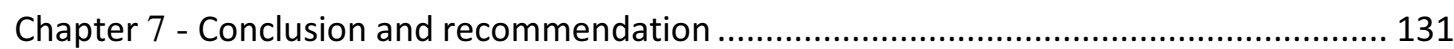

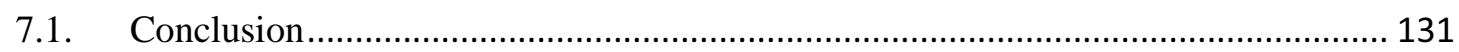

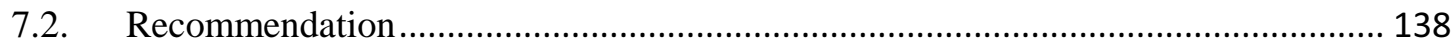




\section{Chapter 1}

\section{Introduction}

\subsection{Introductory Background}

The phenomenon of generating two streams at different temperatures from a vortex tube with single injection was discovered by Ranque in 1930's, and hence was named as Ranque effect. Without any moving parts or chemical reaction within the tube, the phenomenon results only from the fluid dynamic effects.

It is shown in Figure 1.1. that a typical counter-flow vortex tube contains a straight tube with tangential injection, through which compressed gas is injected into the tube, and two exits located at each end of the tube, which allows the streams at different temperatures to be exhausted from the vortex tube. As shown, the tube is completely hollow and there are no other parts inside the tube; hence, the separation of the two streams at different temperatures inside the vortex tube must be based on some fluid dynamic or thermodynamic effects. Other types of vortex tubes will be discussed in the flowing chapter, including the uni-flow vortex tube, which has the cold and hot exists located at the same end of the tube. Since only the counter-flow vortex tube was investigated in this study, hereafter the vortex tube, without any further specific description, will refer to a counter-flow vortex tube.

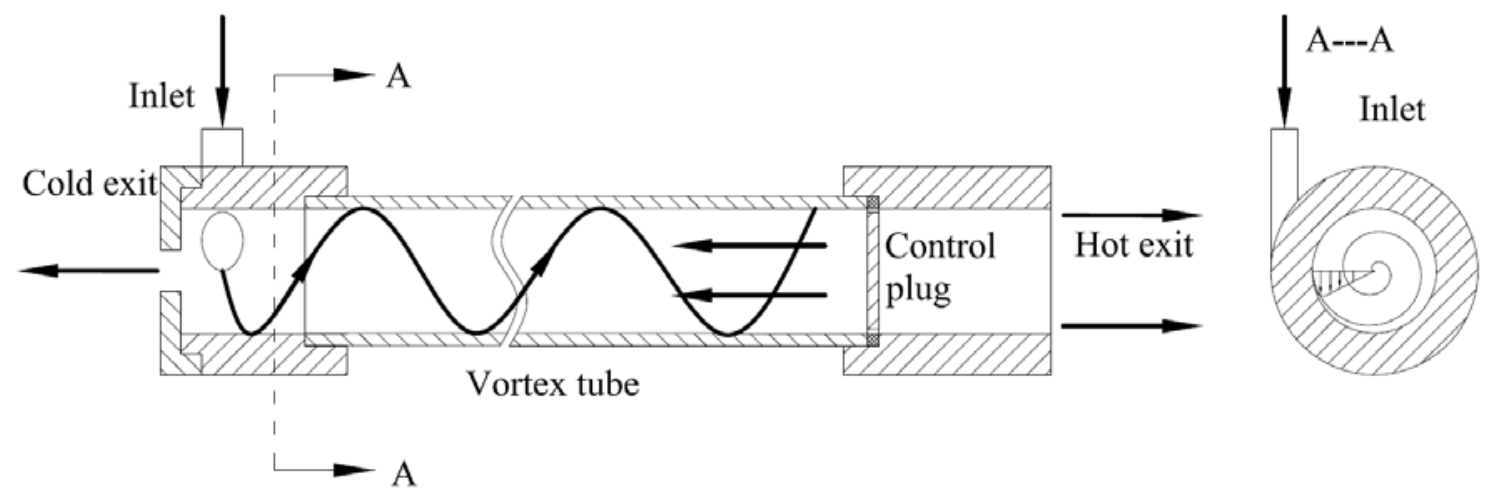

Figure 1.1. Flow structure in a counter-flow vortex tube 
It has been observed that when the compressed gas is injected into the tube tangentially at a high velocity, it starts rotating and moving to the hot end, i.e. the other end from the injection [1]. A small portion of the flow escapes from the gap between the control plug and the tube with higher temperature than at the injection point, which in the literature is referred to as the hot stream. The other part of the flow is then forced back by the control plug and moves to the cold end through the central region of the tube. This central flow is then exhausted from the central exit near the injection point, at a lower temperature than at the injection, and forms the cold stream. Thus, the injected flow is divided into two flows with different temperatures, and this phenomenon is well known as the temperature separation in a vortex tube or Ranque effect $[2,3]$.

The vortex tube was invented by French physicist Georges J. Ranque in 1933 [2]. He found that when compressed gas was injected tangentially into the tube, flow streams at lower and higher temperature were generated and exhausted from different ends of the tube. The cold stream was exhausted from the central exit near the inlet and the hot stream was exhausted from the peripheral exit at the other end of the tube. Ranque explained the separating effect in the vortex tube as one, which depended on expansion and compression. In 1947, the German physicist, Rudolf Hilsch, improved the performance of the tube and published his findings in a highly cited paper [4]. Due to the contributions of different researchers in developing an understanding of the knowledge of the vortex tube, the vortex tube has been referred to be the Ranque Vortex Tube (RVT), the Hilsch Vortex Tube (HVT), and the Maxwell-Demon vortex tube (MDVT). However, in the present time and for consistency in this work, it is referred to as the Ranque-Hilsch Vortex Tube (RHVT).

Since the invention of the tube, many researchers have investigated the vortex tube with the aim of improving the performance of the vortex tube system, exploring the flow structure inside the tube, and identifying the underlying mechanism for the Ranque effect. In the 1960s, Takahama [5] performed a series of experiments and developed several formulas describing the relationships between the different parameters of the vortex tube and the tube performance. Balmer [6], in 1988, using incompressible fluid, i.e., water, as the working material in the vortex tube system, 
found that the separation effect in the vortex tube still existed when the inlet pressure was $103 \mathrm{MPa}$. In recent decades, there have been numerous attempts to use numerical simulations to investigate the complex flow behaviours in the vortex tube [7-12]. Different turbulence models have been employed in simulating the flow inside a vortex tube. A comparison of different turbulence models in predicting the temperature separation in a vortex tube, showed that temperature separation predicted by the standard $k-\varepsilon$ turbulence model, was better correlated to the experimental results [10]. The outcomes of experimental, theoretical and numerical investigations of the vortex tube have been summarised in [13, 14] and the explanations for the temperature separation in the vortex tube have been summarized in a critical review [3], in which a novel hypothesis has been proposed.

Different explanations for the phenomenon of temperature separation in a vortex tube have been proposed. For example, one simple explanation for the temperature separation in relates to the pressure gradient inside the tube. The higher pressure of the peripheral flow and lower pressure of the central flow are the reasons for the generation of higher temperature and lower temperature in the tube due to the effects of compression and expansion, respectively. Another explanation for the thermal separation relates to the energy transferred from the inner flow to the outer flow. The angular velocity of the inner flow is believed to be higher than the flow in the peripheral region; hence the decrease in the angular momentum of the inner flow is transferred as kinetic energy to the outer vortex during the axial movement of the rotating flow. Then the temperature of the outer layer flow increases due to energy gain, and the temperature of the inner flow drops due to the loss of energy. However, none of these explanations covers all the aspects of the thermal separation in the vortex tube. Furthermore, many of the explanations and hypotheses are contradictory [3].

Due to the complex structure of the internal flow, the reasons for generating hot and cold flows from a single injection into a vortex tube have not been identified and the flow behaviour inside the vortex tube, therefore, remains unclear. Aiming to conclude a reasonable explanation for the temperature separation in a vortex tube, this study presents high-fidelity experimental investigations focusing on the flow 
behaviour inside the vortex tube. The contents of this thesis will be introduced later in this chapter.

\subsection{Motivation and significance}

According to the working process described previously, the vortex tube has many advantages compared with other thermal devices, which has no moving parts, no electrical or chemical input, is small, lightweight, low in cost, maintenance free, and produces instant adjustable cold and hot air. These advantages make the vortex tube an attractive device in many applications, including cooling, heating, dehumidification and mixture separation. For example, fluid cooling is applied to remove the heat generated at the cutting zone during machining, especially, during the machining of very hard materials. The cold air, exhausted at high velocity from the vortex tube, provides both cooling and removal of the chips produced during machining. This completely eliminates or significantly reduces the need for liquid coolant, the latter being more complicated, expensive, and environmentally hazardous. The application of the vortex tube in cooling the cutting zone has been investigated to optimise the cooling performance [15-17].

The vortex tube system has also been used to separate mixtures of gases. Peak oxygen purity of $80 \%$ at yields of up to $25 \%$ has been demonstrated from compressed air in a recent report [18], which indicates the potential application of the vortex tube in producing nitrogen and oxygen from a single injection of compressed air.

Williams [19] proposed another potential application of the vortex tube system, i.e. producing ice. He stated that using a vortex tube, the objective of producing ice can be achieved with the additional benefits of producing water and heat, and increasing combustion efficiency and energy storage. The vortex tube has also been used to dehumidify air or generate water from air. Liew et al. [20] reported that the concentration of liquid increases with increasing humidity of the injected gas.

Therefore, it is reasonable to conclude that when factors such as compactness, energy resources, reliability and equipment cost are to be considered, the RHVT 
becomes a feasible device for instant heating and cooling, thermal testing, dehumidification, gas liquefaction, ice production, separation of mixtures, DNA applications and other more general purposes. These significant advantages and wide applications have encouraged scientists and engineers to continue doing more research into the mechanism underlying the vortex tube.

The main limitations of using the vortex tube in industry are its low thermal efficiency, and noise. As concluded in [3, 13], the maximum thermal efficiency of the vortex tube can reach to about $30 \%$, which is lower than other thermal devices. As such, this lower efficiency leads to a narrower industrial application of the vortex tube. Kurosaka [21] reported the acoustic streaming effect in the vortex tube, in which the noise generated by running the vortex tube reached around $125 \mathrm{~dB}$. These drawbacks of the vortex tube may certainly limit its further application.

With the aim of forming an explanation for the temperature separation in the vortex tube, the expected result of this study is obviously important in understanding the flow behaviour, in explaining the thermal separation, and improving the performance of the vortex tube system for wider application. Even if a full explanation is not completed, directions for, and the findings from further research, will benefit from investigations of the flow structure in the tube and clarification of the different factors, which influence the energy transfer between the different flow layers.

\subsection{Publications arising from this thesis}

The research results discussed in this thesis have led to the generation of a number of publications, including international conference articles and journal manuscripts. The international journals to which the work has been submitted are closely related to the field of the research of this dissertation and will be cited in the following section. The publications arising from this research are listed below: 


\section{$\underline{\text { Refereed Journal Articles: }}$}

1. Y. Xue, M. Arjomandi, R. Kelso, Experimental study of the thermal separation in a vortex tube, Experimental Thermal and Fluid Science, 46 (2013), 175-182.

2. Y. Xue, M. Arjomandi, R. Kelso, Experimental study of the flow structure in a counter flow Ranque-Hilsch vortex tube, International Journal of Heat and Mass Transfer, 55(21-22) (2012) 5853-5860.

3. Y. Xue, M. Arjomandi, R. Kelso, Visualization of the flow structure in a vortex tube, Experimental Thermal and Fluid Science, 35 (2011) 1514-1521.

4. Y. Xue, M. Arjomandi, R. Kelso, A critical review of temperature separation in a vortex tube, Experimental Thermal and Fluid Science, 34 (2010) $1367-1374$.

\section{Journal Papers submitted:}

5. Y. Xue, M. Arjomandi, R. Kelso, Energy analysis within a vortex tube, Experimental Thermal and Fluid Science, (Submitted).

6. Y. Xue, M. Arjomandi, R. Kelso, The working principle of a vortex tube, International Journal of Refrigeration (Submitted).

\section{Refereed Conference Articles:}

1. Y. Xue, M. Arjomandi, R. Kelso, Experimental study of the flow structure in a vortex tube, The 7th Australasian Congress on Applied Mechanics, Adelaide, Australia, 2012.

2. Y. Xue, M. Arjomandi, R. Kelso, Experimental study of the thermal separation in a vortex tube, 18th Australasian Fluid Mechanics Conference, Launceston, Tasmania, Australia, Dec., 2012. 
3. Y. Xue, M. Arjomandi, R. Kelso, Flow visualization to determine the flow structure in a vortex tube, 17th Australasian Fluid Mechanics Conference, Auckland, New Zealand, 2010.

4. Y. Xue, M. Arjomandi, Thermal investigation of the Ranque-Hilsch vortex tube in: 2009 Interdisciplinary Conference on Chemical, Mechanical and Materials Engineering (2009 ICCMME), Melbourne, Australia, 2009.

\subsection{Preview of this thesis}

As introduced in section 1.1, different aspects of the vortex tube have been investigated since the invention of this device. Several hypothesises have been proposed to explain the temperature separation in the vortex tube. However, due to the complex flow condition inside the tube, there have been a number of explanations for the temperature separation phenomenon occurring within the vortex tube. This thesis presents a fundamental investigation on the Ranque-Hilsch Vortex Tube, aiming to identify and define the dominant factors responsible for the thermal separation. This study includes a critical review of the current explanations, with regard to the design of the experiment procedures, visualization of the flow behaviour, accurate measurements of the flow properties inside the tube, and analysis based on the experimental data. Such parameters are reviewed in this thesis with regard to providing a greater understanding of the flow behaviour and other factors which contribute to the process of temperature separation.

This thesis is a collection of manuscripts that have been published, accepted for publication or are currently under review in international peer-reviewed journals. These publications cover the progress made in the course of this study, which includes the development, validation and application of the hypothesised explanation for the temperature separation in a vortex tube. This section describes how the individual journal articles are linked together to achieve the objectives related to the research topic.

Chapter 2 covers previous and contemporary literature regarding the vortex tube, including the numerous theoretical, experimental and numerical investigations. 
The research gaps are identified, and the objectives in the present study with reference to the previous literature are introduced in chapter 2.

The research direction for this study has been identified from the analyses of the current explanations, which are presented in Chapter 3. In this published article, a review of prior explanations for the mechanism of temperature separation in a vortex tube is introduced and the effects of different factors are critically analysed and evaluated. A key knowledge gap raised within the critical review relates to the mechanism of energy transfer between different layers of flow d. A conceptional explanation has been proposed in this study relating to the fact that pressure gradient near the injection and partial stagnation of the multi-circulation (the supposed flow structure near the hot end of a vortex tube) are the mechanisms underlying the temperature drop and rise, respectively. With the analysis results from this published article, the research approach is divided into several parts, including the confirmation of the flow behaviour inside the tube, the clarification of the energy transfer between different layers of flow, and the application of the proposed explanation in explaining the working process and geometric effects within a vortex tube system.

Chapter 4 reports on an experimental study of the flow structure inside a vortex tube, using flow visualization in a water-operated vortex tube, with the aim to verify the proposed flow behaviour as was described in the previous article. Return of the peripheral flow near the injection, and the proposed flow structure of the multi-circulation near the hot end, were both observed via different visualisation methodologies [22]. Velocity profiles inside the tube obtained from the visualization results provide quantitive evidence for the proposed flow structure. The volume flow rate along the tube, calculated from the axial velocity profiles, also indicates the existence and location of the proposed cold core and multi-circulation zones. The published manuscript supports the proposed hypothesis for the temperature separation in a vortex tube, by confirming the supposed flow structure with visualization results. As an actual vortex tube uses compressible gas as the working material, the following articles report the experimental studies in an air-operated vortex tube. 
Chapter 5 consists of two journal papers, which firstly focus on the flow structure, and secondly the thermal separation inside an air-operated vortex tube. The flow properties, including the three dimensional velocity distribution, pressure and temperature gradients, were measured in a large-scale vortex tube. The first paper [23] reports the influence of geometrical parameters on the tube performance, upon which optimisation of the tube performance, was performed. These geometrical effects are further discussed in the following chapter, which covers the validation of the proposed explanation. A transformation from a forced vortex structure at the cold end, to an irrotational vortex near the hot end, was observed in this study based on the velocity measurements, and also supported by the measured static pressure gradient. The observed swirl velocity profiles, agreeing with the outcomes in chapter 4 , and confirm the proposed flow structure presented in this thesis.

Further analysis of the flow structure, based on detailed measurements of the flow properties, is presented in the second paper. The proposed explanation is supported by confirming the supposed flow structure inside the air-operated vortex tube, particularly with regard to the axial and radial velocity profiles. In addition, the measurement results of the pressure, temperature and density profiles agree well with the hypothesised explanation. A novel analysis of exergy density inside the airoperated vortex tube is performed in the paper for the first time, and this generates significant evidence for the postulated explanation, by clarifying the energy transfer between different layers of flow.

The experimental results reported in this chapter further support the proposed explanation, by providing significant evidence confirming both the flow structure and clarifying the energy transfer process. The proposed explanation can be further validated using the experimental data from other reports, and the theoretical analysis of the working process plays an important role in the validation of the hypothesis. This will be presented in Chapter 6 .

The first manuscript contained in Chapter 6 reports a finalised explanation of the temperature separation in detail together with further exergy analysis based on other experimental studies. A good agreement between the exergy profiles of current 
research and other experimental reports indicates the acceptance and effectiveness of this methodology, as well as the proposed explanation. The second manuscript provides evidence for the explanation, including the confirmation of the flow structure, and the exergy analysis inside a vortex tube. Comparison between the theoretical estimated temperature drop based on the forced vortex assumption, and the actual experimental results, provides positive support for the hypothesis forwarded in this study. According to the proposed explanation, the influence of the geometrical parameters on the vortex tube performance has been analysed and has been shown to have a good agreement with the published experimental results. Hence, the validation of the proposed explanation for the temperature separation in a vortex tube is further substantiated by the comparison of experimental data from other investigations, and theoretical analysis.

Conclusions of this research and recommendations for future investigations are presented in chapter 7 . 


\section{Reference}

[1] Exair, http://www.exair.com/vortextube/vt.

[2] G.J. Ranque, Experiments on expansion in a vortex with simultaneous exhaust of hot air and cold air, J Phys Radium, 4 (1933) 112.

[3] Y. Xue, M. Arjomandi, R. Kelso, A critical review of temperature separation in a vortex tube, Experimental Thermal and Fluid Science, 34 (2010) 1367-1374.

[4] R. Hilsch, The use of the expansion of gases in a centrifugal field as cooling process, Review of Scientific Instruments, 18 (1947) 108-113.

[5] H. Takahama, Studies on vortex tubes, Bull. JSME, 8 (1965) 433-440.

[6] R.T. Balmer, Pressure driven Ranque Hilsch temperature separation in liquids, Journal of Fluids Engineering, Transactions of the ASME, 110 (1988) 161-164.

[7] T. Farouk, B. Farouk, Large eddy simulations of the flow field and temperature separation in the Ranque-Hilsch vortex tube, International Journal of Heat and Mass Transfer, 50 (2007) 4724-4735.

[8] U. Behera, P.J. Paul, K. Dinesh, S. Jacob, Numerical investigations on flow behaviour and energy separation in Ranque-Hilsch vortex tube, International Journal of Heat and Mass Transfer, 51 (2008) 6077-6089.

[9] A. Secchiaroli, R. Ricci, S. Montelpare, V. D'Alessandro, Numerical simulation of turbulent flow in a Ranque-Hilsch vortex tube, International Journal of Heat and Mass Transfer, 52 (2009) 5496-5511.

[10] T. Dutta, K.P. Sinhamahapatra, S.S. Bandyopdhyay, Comparison of different turbulence models in predicting the temperature separation in a Ranque-Hilsch vortex tube, International Journal of Refrigeration, 33 (2010) 783-792.

[11] A.R. Bramo, N. Pourmahmoud, Computational fluid dynamics simulation of length to diameter ratio effects on the energy separation in a vortex tube, Thermal Science, 15 (2011) 833-848.

[12] T. Dutta, K.P. Sinhamahapatra, S.S. Bandyopadhyay, Numerical investigation of gas species and energy separation in the Ranque-Hilsch vortex tube using real gas model, International Journal of Refrigeration, 34 (2011) 2118-2128.

[13] S. Eiamsa-ard, P. Promvonge, Review of Ranque-Hilsch effects in vortex tubes, Renewable and Sustainable Energy Reviews, 12 (2008) 1822-1842.

[14] M. Yilmaz, M. Kaya, S. Karagoz, S. Erdogan, A review on design criteria for vortex tubes, Heat and Mass Transfer/Waerme- und Stoffuebertragung, 45 (2009) 613-632. 
[15] B. Yalçin, A.E. Özgür, M. Koru, The effects of various cooling strategies on surface roughness and tool wear during soft materials milling, Materials and Design, 30 (2009) 896-899.

[16] A. Ahmad-Yazid, Z. Taha, I.P. Almanar, A review of cryogenic cooling in high speed machining (HSM) of mold and die steels, Scientific Research and Essays, 5 (2010) 412-427.

[17] M. Selek, S. Tasdemir, K. Dincer, S. Baskaya, Experimental examination of the cooling performance of Ranque-Hilsch vortex tube on the cutting tool nose point of the turret lathe through infrared thermography method, International Journal of Refrigeration, 34 (2011) 807-815.

[18] A.M. Crocker, G.L. Sutphin, D.V. Cassisi, C. Knowlen, R.F. Weimer, Investigation of enhanced vortex tube air separators for advanced space transportation, in: 40th AIAA/ASME/SAE/ASEE Joint Propulsion Conference and Exhibit, Fort Lauderdale, FL, 2004.

[19] D.T. Williams, Ranque-Hilsch Vortex Tube for Refrigeration in Developing Communities, in, dissigno, San Francisco, CA USA, 2005.

[20] R. Liew, W.R. Michałek, J.C.H. Zeegers, J.G.M. Kuerten, Droplet behaviour in a Ranque-Hilsch vortex tube, Journal of Physics: Conference Series, 318 (2011).

[21] M. Kurosaka, Acoustic streaming in swirling flow and the Ranque-Hilsch (vortex-tube) effect, Journal of Fluid Mechanics, 124 (1982) 139-172.

[22] Y. Xue, M. Arjomandi, R. Kelso, Visualization of the flow structure in a vortex tube, Experimental Thermal and Fluid Science, 35 (2011) 1514-1521.

[23] Y. Xue, M. Arjomandi, R. Kelso, Experimental study of the flow structure in a counter flow Ranque-Hilsch vortex tube, International Journal of Heat and Mass Transfer, 55 (2012) 5853-5860. 


\section{CHAPTER 2}

\section{Literature review}

\subsection{Introduction}

The generation of two streams at different temperatures from the vortex tube was discovered in 1930's by Georges J. Ranque. Since then it has been known as the Ranque effect and has been a popular research topic within the scientific community. Ranque proposed the compression and expansion effects as the underlying reasons for the process of temperature separation in a vortex tube [1,2]. Later, the geometrical parameters and performance optimisation of the tube were further investigated by Rudolf Hilsch [3].

Since Ranque's discovery of the tube in 1933, the vortex tube has been investigated experimentally, theoretically and numerically, aiming to identify the dominant factors for the thermal separation phenomenon and to improve tube performance. Investigations of the vortex tube have been focused on several aspects, such as exploration of the thermal separation, optimisation of the geometrical parameters, theoretical analysis of the thermal separation, and numerical simulation of the flow process, etc. In 1954, the research history of the tube was summarised by Westley [4] and a total of 116 publications were analysed in his paper. Other reviews on the investigation of the vortex tube and its applications were published by Curley and McGree [5], Kalvinskas [6], Dobratz [7], Nash [8], Soni [9], Hellyer [10], Gustol [11] and Leont'ev [12]. In 2008, Smith and Pongjet [13] published a detailed review of the vortex tube, in which experimental, theoretical and numerical investigations were summarized. Yilmaz et al. [14] published another review in 2009, in which experimental parameters and design criteria were summarised.

The previous investigations of the vortex tube are summarized in the following sections, including the type of the vortex tube, the working medium, the geometry of the tube, the flow visualization results, and the numerical simulation. The knowledge 
gaps in the previous research and the objectives of this study are addressed in this chapter.

\subsection{Statement of research}

\subsubsection{Different types of vortex tube}

In the investigation of the vortex tube, different types of the tube have been studied. The main types of vortex tube include; the counter-flow vortex tube, uniflow vortex tube, conical vortex tube; double-circuit vortex tube, two-stage vortex tube, and a vortex tube in different surroundings, such as an insulated vortex tube, a non-insulated vortex tube, and a vortex tube with cooling water around.

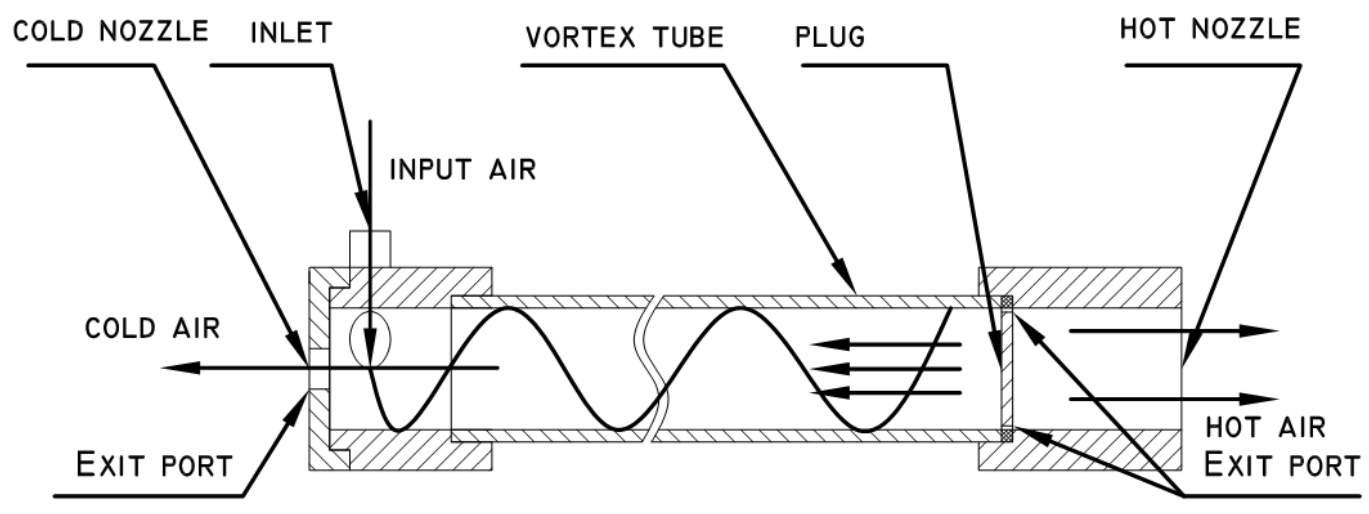

Figure 2.1. Airflow structure in a counter-flow vortex tube

As shown in Figure 2.1, a standard counter-flow vortex tube consists of a cold nozzle from which cold air is exhausted; a vortex chamber with tangential injection, a long straight tube, in which temperature separation happens, and a peripheral hot nozzle from which hot air exits. Passing through the vortex chamber, the tangentially-injected compressed air forms a strong vortical flow in the vortex chamber. The vortical airflow, which is moving towards the hot end, is later forced back by a plug placed at the hot end of the tube. The plug adjusts the balance between the rate of air escaping peripherally from the hot exit, and that being forced back to the cold end. A counter-flow vortex tube can be divided into two parts, i.e. the cold part and the hot part, due to the flow temperature gradient within the tube. 
The cold part is located near the injection point and the hot part is located at the far end of the tube.

The structure of a standard uni-flow vortex tube is shown in Figure 2.2. Having similar structure to a counter-flow vortex tube, a uni-flow vortex tube has two exits at the same end. When the swirling flow moves to the exit end, the peripheral part of the flow is exhausted from the peripheral gap between the plug and the wall of the tube, while the central part of the flow is exhausted from the cold nozzle at the same end. The uni-flow vortex tube is found to have lower efficiency than the counterflow vortex tube, due to the stronger mixing effect of the cold and hot flows near the exit [13].

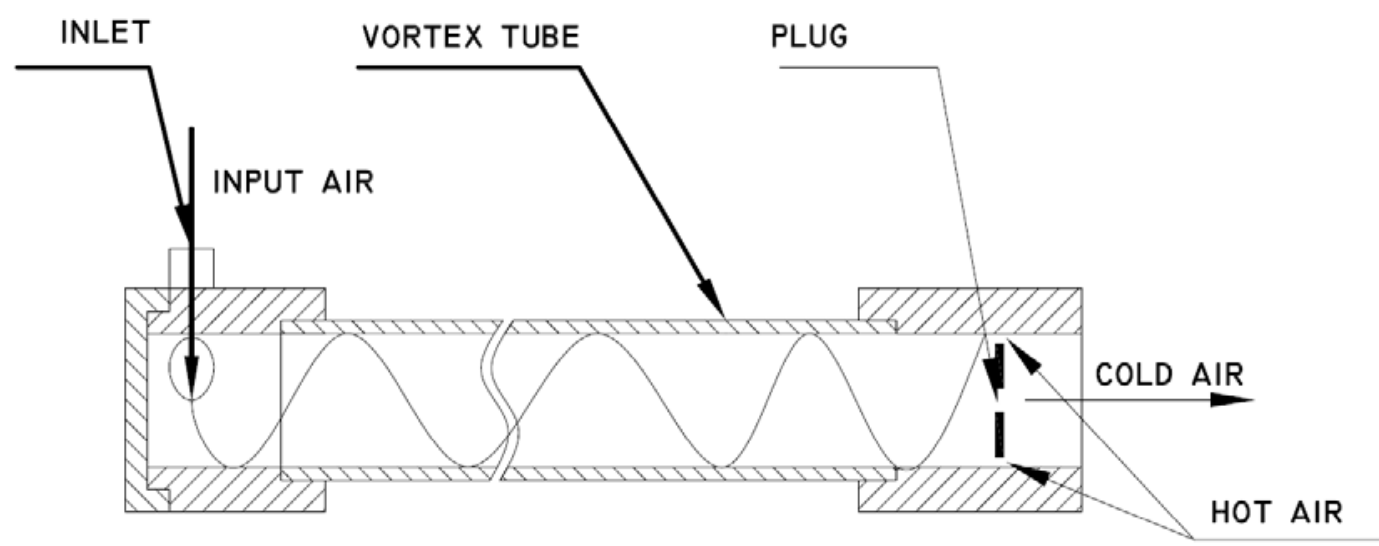

Figure 2.2. Airflow structure in a counter-flow vortex tube

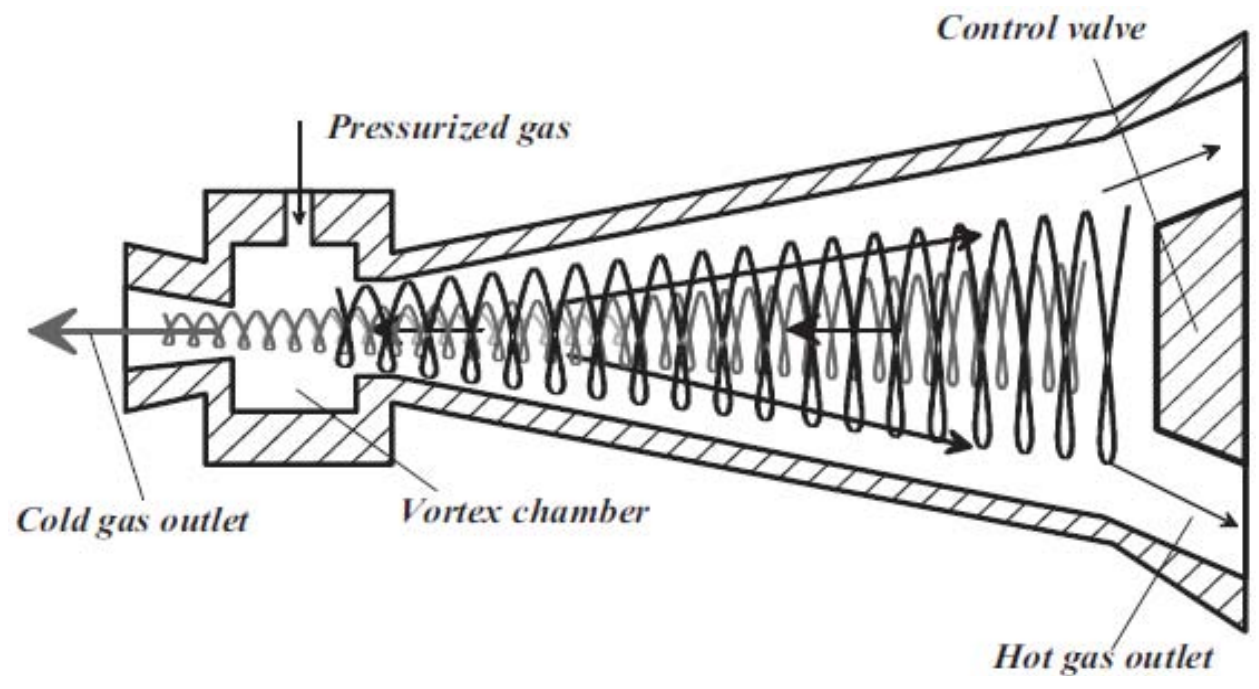

Figure 2.3. Structure of a conical vortex tube [15] 
In the optimisation of the vortex tube, a short conical vortex tube was found to be effective in shortening the tube length, as shown in Figure 2.3. In 1961, Paruleker reported that a small parameter $L_{v t} / D_{v t}$ of 3 was achieved, by varying the conical angle of the vortex tube [15]. In order to shorten the tube length, Takahama introduced the divergent vortex tube in 1981. This was identical to the conical vortex tube as reported in [16], and was reported to exhibit the same performance parameters as the normal tube, but with a smaller length. The conical vortex tube has also been investigated by Poshernev et al. with regard to other chemical applications [17-22].

In 1996, Piralishvili and Polyaev [23] introduced a new type of vortex tube (Figure 2.4), termed the Double-Circuit vortex tube, with a conical tube to improve the performance. At the hot end, there was an orifice which allowed feedback gas to be injected into the vortex tube in the centre of the control valve. The feedback gas had the same temperature as the inlet gas but was introduced at a lower pressure. With this design, the cooling power of the system was increased and the performance of the vortex tube was claimed to be improved.

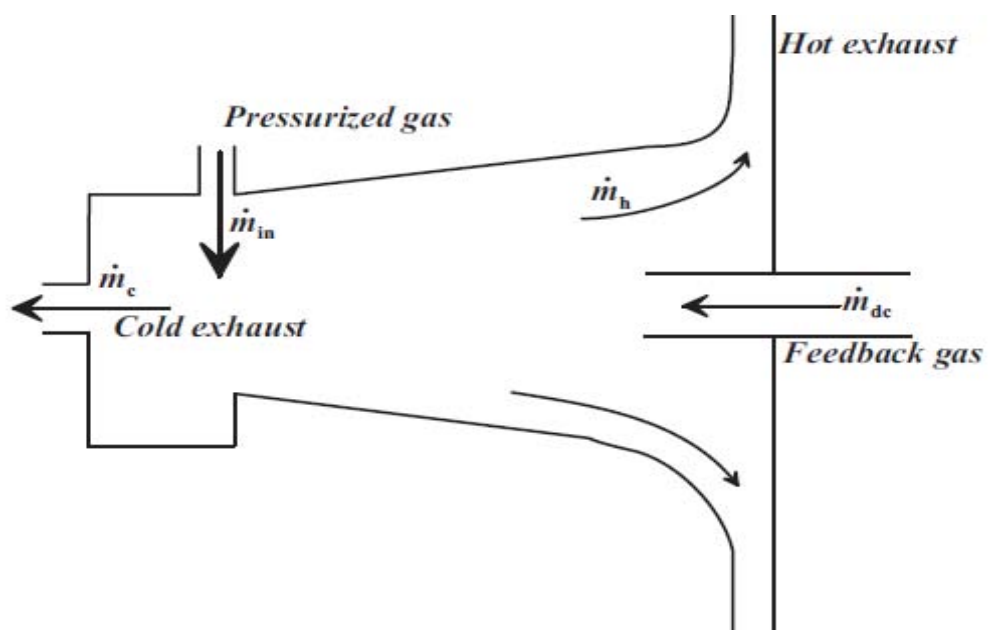

Figure 2.4. Structure of the Double-Circuit vortex tube [23]

The effect of the cooling on the vortex tube was studied by Eiamsa-ard et al. [24]. Cold water was placed outside the tube and heat transfer from the hot tube to the cold water was counted in their investigation. As the result, an increase in the temperature drop and the cooling efficiency of the vortex tube was found when cold water was applied outside of the tube as a cooling material. 
A two-stage vortex tube system was reported in 2001 by Guillaume and Jolly [25]. In their study, the outlet of cold air form the first vortex tube was connected to the injection port of a second vortex tube. They reported that the temperature difference at each stage was greater than that generated by the single stage vortex tube under the same operating conditions.

Similar multi-stage vortex tube systems have been investigated to optimise the performance of the system. Threefold type and six-cascade type vortex tube systems, which consisted of three and six vortex tubes connected in series, respectively, were investigated by Dincer [26]. They reported an improvement of the system performance when the number of connected vortex tubes was increased, with the sixcascade vortex tube system generating the maximum temperature drop.

In contrast to the straight tube, a new type of vortex tube has been reported, in which the main tube is curved as shown in Figure 2.5. The influence of the uniform curvature of the main tube on the vortex tube performance was analysed by Valipour and Niazi [27] in 2011. It was found that the extent of the temperature difference between the two exhausted streams, differed depending on the extent of vortex tube curvature, with the maximum refrigeration capacity occurring with the 110 degree curved vortex tube. However the maximum temperature difference was generated by the straight vortex tube.

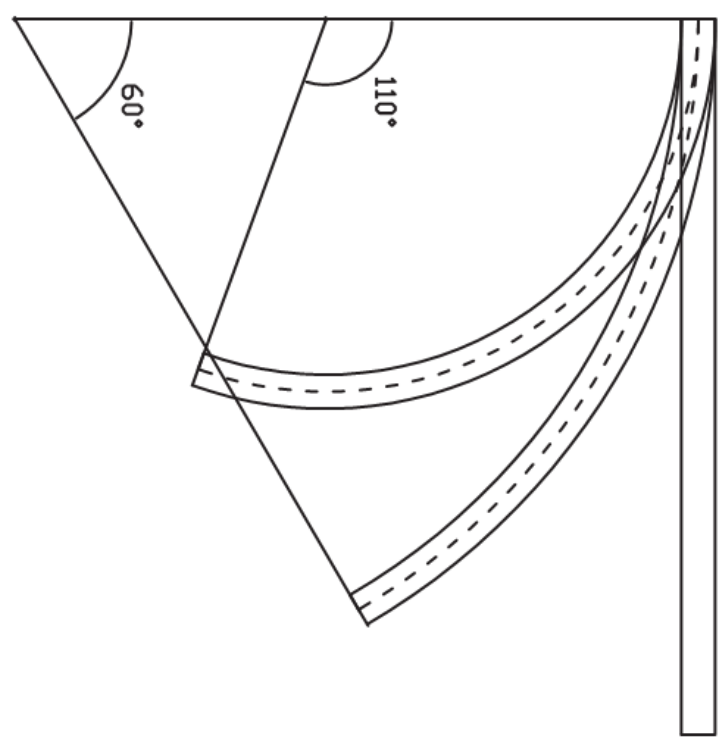

Figure 2.5. Curved vortex tubes [27] 
There are also other types of vortex tube system that have been investigated, including the triple-stream vortex tube, the self-evacuating vortex tube, and the vortex ejectors [14]. These tubes are being investigated or used in specific fields, and will not be fully discussed in this thesis.

\subsubsection{Working medium}

Different working medium have been successfully applied in the vortex tube, including, compressed air, oxygen, methane, and other gas mixtures. The influence of the different materials, including both gases and liquids, used in the vortex tube has been studied and will be introduced in this section.

Due to the different temperature of the exhausted fluid from the exits, the vortex tube is found applicable for separating various gas mixtures. The first studies on the separation of mixtures with the RHVT were reported in 1967 by Linderstrom-Lang [28], and later by Marshall [29]. Different gas mixtures, including oxygen and nitrogen, carbon dioxide and helium, carbon dioxide and air, as well as other mixtures, have been used as the working medium inside the vortex tube. The separation effect was found to be a function of both the cold flow ratio, and the geometrical parameters. The influence of different working mediums on the tube performance has been further investigated and reported [30-33], with the media including air, oxygen, nitrogen, and argon. The successful applications of the vortex tube in gas mixture separation [34], fluid concentration [35], and gas liquefaction [22, 36], have also been reported. These successful applications show the diversity of gas, and gas mixtures, which can be used in the vortex tube.

A two-phase steam, consisting of both gas and liquid phase water, has been tested in a vortex tube [37]. The percentage of the gaseous component in the mixture was reported to be an important parameter in generating the temperature difference between the two exhausted streams. In 1979, two-phase propane [38] was used in the vortex tube as the working medium. It was stated that a significant temperature difference between the two outlets could be achieved when the dryness of the liquid and gaseous propane was higher than 0.8 . 
Liquid, as the working material in a vortex tube, was injected into the tube at high inlet pressure $(103 \mathrm{MPa})$, and the rise in the temperature of the exhausted water from both exits was reported [39]. In contrast, when a low pressure was set at the inlet, no measurable temperature difference between the two exhausted water flows from the vortex tube was reported [40]. Hence, the Ranque effect does not exist when liquid is used inside the vortex tube. Instead, temperature of the working medium rises when high pressure is set at the inlet.

In conclusion, compressible gases can be used as the working medium inside a vortex tube for the successful operation of a vortex tube system. When a two-phase flow is employed, the high extent dryness of the injected stream, which leads to the stream performing as a gas, ensures the generation of significant temperature difference. Liquid cannot be used in a vortex tube for generating the Ranque effect, i.e., hotter and cooler streams from the same injection. Therefore, compressed air has been selected in this research and the use of water in the study described is only aimed at gaining a better understanding of the flow behaviour within the vortex tube.

\subsubsection{Geometry of the tube}

In the investigations on the vortex tube, the tube performance has been found to be sensitive to the geometrical parameters, including the size and shape of the control plug, the size and shape of the injection port, the diameter and length of the tube, the structure of the vortex chamber, the diameter of the cold and hot nozzles, among other factors. Many investigations on these geometrical parameters have been reported, with the aim of identifying the primary factors underlying the energy separation, and also with regard to optimise the performance of the vortex tube system.

Studies on the effect of the tube geometry were started by Hilsch [3] in 1947. Based on his experimental results, the friction between the peripheral and internal gas layers was proposed as the reason for the temperature rise within the vortex tube, and these correlated with his experimental results obtained. Later, the influence of the geometry of the vortex tube system on its performance was optimised 
experimentally [41]. It was found that the optimum operation of the vortex tube could be described by a relationship between the injection area, the tube length and diameter, the cold and hot exits characteristics, and the inlet pressure.

A series of papers on the RHVT were published since the 1960s by Takahama $[16,37,42-47]$, reported on the various relationships between the different parameters of the vortex tube and the optimum tube performance. Similar research on the geometrical effects was reported by Soni [48], and in his work the relationships between the design parameters for an optimum vortex tube were proposed.

In a vortex tube, the mass flow rate of the injection and the formation of the vortex flow are generally dictated by the inlet nozzle. Investigations on the injection port, which mainly concern the shape, size and number of the inlet nozzles, have been reported. Considering the conventional tangential injection port, a new inlet nozzle with equal Mach number gradient and an intake flow passage with equal flow velocity was designed and tested in a modified vortex tube by Wu et al. [49] (Figure 2.6.). The experimental results indicated that the cooling effect of the improved nozzle was about $2.2{ }^{\circ} \mathrm{C}$ lower than that of the normal rectangular nozzle, and $5{ }^{\circ} \mathrm{C}$ lower than that of the nozzle with an Archimedes' spiral design.

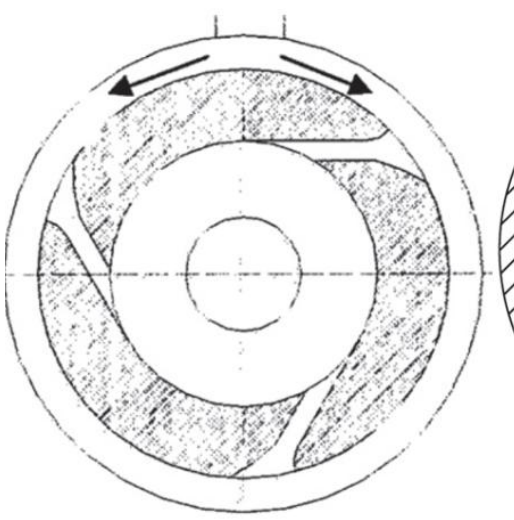

Conventional nozzle

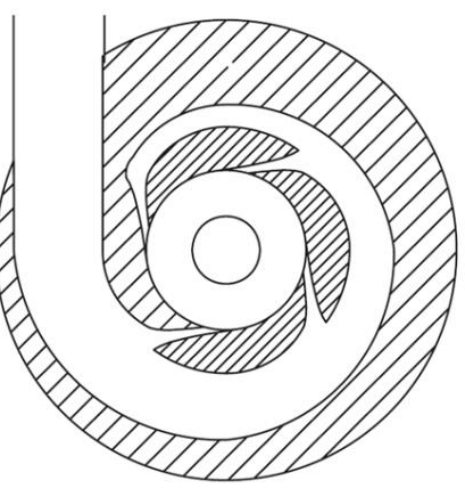

Nozzle proposed by Wu et al.

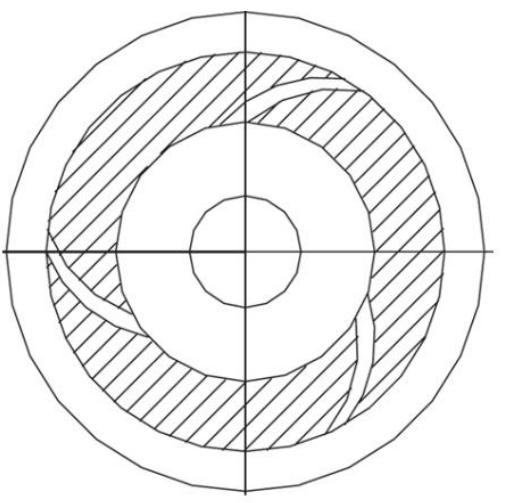

Nozzle of Archimedes'

Figure 2.6. Different inlet nozzles in the vortex tube

Eiamsa-ard [50] reported an investigation of various geometrical parameters, including a different number of the snail entries, and reported that an increase in the nozzle number and the supply pressure led to a rise in the vortex intensity and thus 
the energy separation in the vortex tube. Similar observations have been reported in other publications [51-53].

The hot end control plug is an important component influencing both the cold and the hot mass flow rates. The influence of the control plug dimensions on the tube performance has been reported, and it was shown that the cold mass flow ratio is dictated by the control plug, and is a determining factor of the temperature of the respective exhausted streams [54]. Moreover, the shape of the control plug has been reported not to be a significant component in the performance of the RHVT due to the small differences observed with different plugs [55] (Figure 2.7).

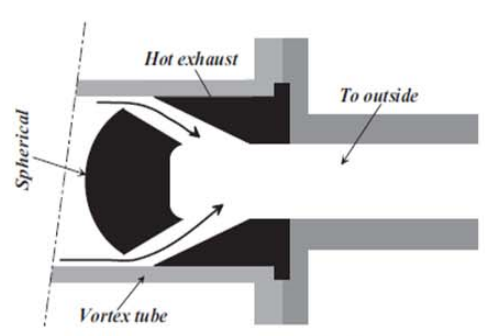

(a) Spherical

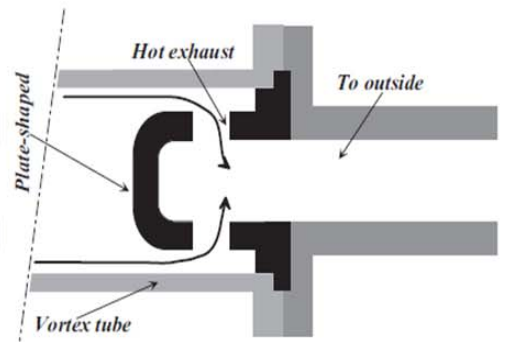

(b) Plate-shaped

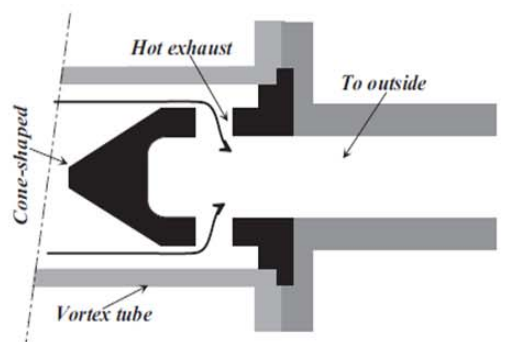

(c) Cone-shaped

Figure 2.7. Investigation of the effect of the control plug [55]

A detwister is a form of vortex stopper, which can be used to block the vortex motion at the exhausts. The applications of the detwister in a vortex tube were reported by Dyskin [56], who reported positive effects on the tube performance. To improve the tube performance, a diffuser, which exhibits similar effects as the detwister in stopping the vortex motion, has also been placed in the vortex tube. It was reported that the application of a diffuser after the hot exit, improved the temperature rise at the hot end and decreased the temperature drop at the cold end [49].

As introduced in previous section, the application of a conical vortex tube can shorten the effective tube length in generating the significant temperature difference from the vortex tube. Optimisation of the conical vortex tube has been performed by varying the conical angle and it has been reported that the vortex tube with a conical angle of $2.3^{\circ} \mathrm{C}$ surpassed a conventional straight tube by an amount of between $20 \%$ - $25 \%$ in its thermal efficiency [57]. Recently, when different inlets were tested, a 
conical angle of $4{ }^{\circ} \mathrm{C}$ was reported as the optimum angle for a divergent vortex tube $[58]$.

A new parameter in the investigation of the vortex tube, named the vortex angle, was studied experimentally by Xue and Arjomandi [59]. The influence of the vortex angle on the performance of the tube was plotted, and an optimum value of the vortex angle was reported in that study. Further investigations on the vortex angle have been conducted, and a similar result was reported that an increase in the vortex angle had negative effects in forming the strong vortex motion, and in generating a significant temperature difference from the tube [60-62].

The sizes of known vortex tubes vary over large range. For example, a vortex tube employed in experimental studies is generally larger than the commercial tubes, with a comparison between $60 \mathrm{~mm}$ and $10 \mathrm{~mm}$ in diameter, respectively, and 2000 $\mathrm{mm}$ to $50 \mathrm{~mm}$ in length, respectively. Moreover, a micro-size vortex tube, with an inner diameter of 2 millimetres, has been tested. It was reported that the temperature distribution inside the tube, and the influence of the geometrical parameters, such as length of the tube and diameter of the exits, were found to be similar to that in a larger vortex tube [63].

These geometrical parameters have been also investigated by many researchers including, Simoes-Moreira [64], Behera et al. [65, 66], Skye et al. [67], Frohlingsdorf and Unger [68], Aydin et al. [60, 69, 70], Arbuzov et al. [71], Farouk and Farouk [72, 73], Saidi et al. [74, 75], Dincer et al. [26, 31, 51, 76, 77], Muller and Nimbalkar [78], Singh et al. [79], Eiamsa-ard et al. [13, 24, 50, 80-82], Kazantseva et al. [83], Ahlbom et al. [84-86], Aljuwayhel et al. [87], Im and Yu [88], Xue and Arjomandi [59], Hamdan et al. [89]. These investigations have all played an important role in the exploration of the physical phenomena in the vortex tube.

The relationships between the geometrical parameters and the tube performance reported in the previous investigations are generally concluded based on experimental or numerical results. As such, there has not been a satisfactory explanation for the temperature difference between the two exhausted streams generated from a vortex tube, which covers all these geometrical effects on the tube 
performance. This issue has been addressed in this study and will be presented in chapter 6 of this thesis.

\subsubsection{Flow visualization}

To explain the Ranque effect in a vortex tube, a good understanding of the flow behaviour inside the tube is essentially required. Flow-visualization techniques have been used to investigate the flow field within the vortex tube, such as dyes and smoke injection. With the injection of dyes or smoke, all subsequent investigations concentrated on tracking the flow trail on the wall near the ends of the tube, and used a clear tube as the main part of the vortex tube for tracking the visual elements. The studies which focused on flow visualization within the vortex tube are summarised in this section.

In 1959, the surface trace on the tube wall was observed by injecting water into a transparent RHVT system [90]. Similar flow visualization of the surface trajectory along the clear vortex tube was also observed by Aydin and Baki [69] as presented in Figure 2.8. However, the difference between the flow trace in the flow visualization and that derived by numerical simulations (figure 2.2.5-4), which will be discussed below, indicates the requirement of further investigation on the flow structure within the vortex tube.

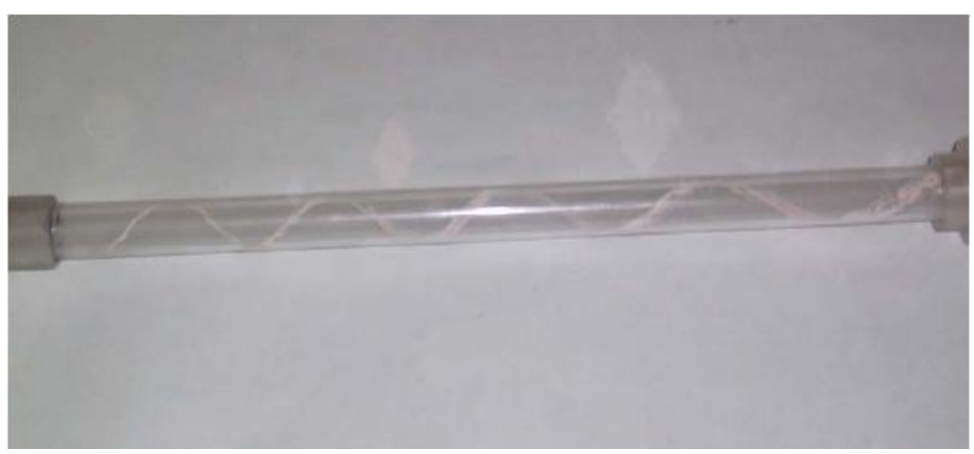

Figure 2.8. Result of the flow visualization [69]

A different visualization result of the surface trajectory along the inner wall of a transparent vortex tube is presented in Figure 2.9. It was reported that the observed sudden change of the trajectory on the vortex tube wall may indicate the existence of 
a partial stagnation point in the vortex tube, however it is difficult to establish the exact position of such a stagnation point [91].

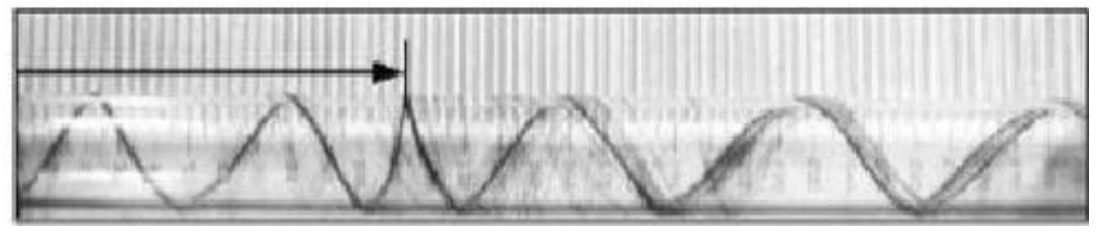

Figure 2.9. Surface trajectory on the wall of the vortex tube [91]

The internal flow pattern of a vortex tube is another aspect, which could be studied using flow visualization. However, due to the strong swirling motion and high turbulence intensity, it is difficult to generate clear visual results for further analysis. Coloured liquid [92], mixtures of powdered carbon and oil [93], and smoke $[94,95]$ have been employed to visualize the flow field within the vortex tube. Unfortunately, none of these visualization techniques were particularly successful. Another attempt of visualizing the internal flow pattern was performed by Piralishvili and Polyaev [23], who used the mixture of kerosene and air with the mass ratio of 1:30. However, the visualized flow pattern obtained was not clear enough to be conclusive.

The structure of the vortical double helix, which is similar to the helix within the vortex tube, was visualized by the method of Hilbert dichromatic filtering [71] (Figure 2.10). The formation of an intense vortex braid near the axis was firstly observed. The authors concluded from the visualization results that the physical mechanisms responsible for the energy separation were viscous heating of the gas due to friction in a thin boundary layer at the walls of the vortex tube, and the adiabatic cooling of the gas at the center. As indicated in the figure, the visual results are still not clear enough for accurate analysis of the flow behavior within the vortex tube. 

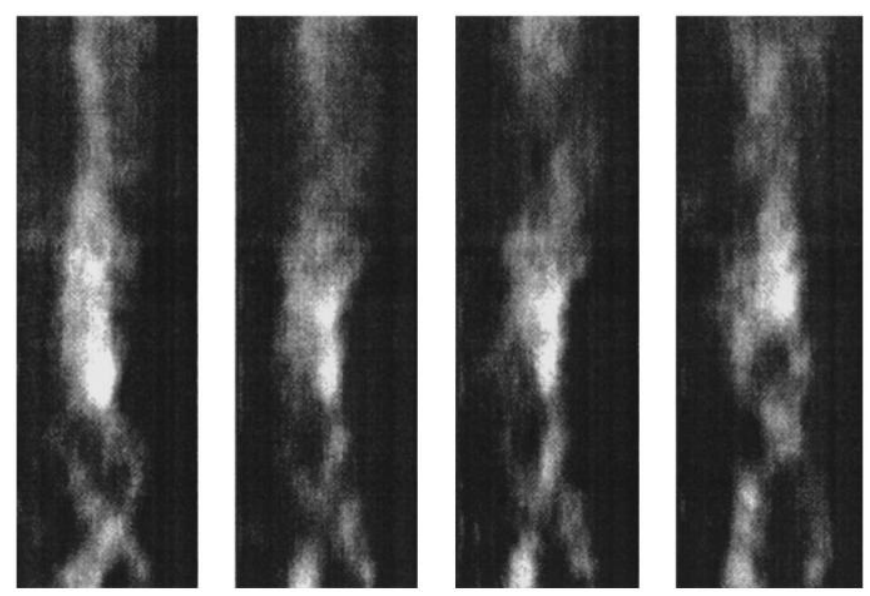

Figure 2.10. Visualization of large-scale vortex in the form of a double helix [71]

The abovementioned visualization techniques have the advantage that it is very easy to qualitatively determine the flow field inside the tube. The disadvantage is that it is not possible to obtain quantitative information about the flow and to determine the temperature field inside the tube. Moreover, according to the above discussed flow visualization results, it is reasonable to conclude that it is difficult to generate clear visual results of the internal flow field.

To produce useful visualization results, the research presented in this study used water as the working medium within a transparent vortex tube. Flow structure inside the tube was observed via new visualization materials, which will be presented in chapter 4.

\subsubsection{Numerical simulation}

The method of numerical simulation has also been employed in the investigation of the vortex tube. The numerical analysis presents a clear description of the flow behaviour and predicts the temperature distribution accurately. Many numerical investigations have shown a strong correlation with the experimental results.

A short summary of the numerical research work is discussed below, using different models based on Computational Fluid Dynamics package (CFD) and Matlab codes. A CFD model of the air flow in a vortex tube was developed by 
Behera et al. [65] and the results were compared with experimental results for the same flow case. The flow inside the tube was explained as a free vortex in the periphery, and a forced vortex in the core. Their further investigations based on the CFD model suggested a different description of the internal flow, which initially involved the cold air being heated up and then cooled down on its way back to the cold end in a counter-flow vortex tube [66]. Frohlingsdorf and Unger [68] studied the phenomena of velocity and energy separation inside the vortex tube using CFX with the $k$ - $\varepsilon$ turbulence model. Friction between inner and outer flow layers was stated as the main reason for energy separation. Eiamsa-ard and Promvonge [80-82] introduced a mathematical model for the simulation of a strongly swirling compressible flow in a vortex tube by using an algebraic Reynolds stress model and the $k$ - $\varepsilon$ turbulence model to investigate the flow characteristics and energy separation in a vortex tube. It was found that a temperature separation in the tube existed and predictions of the flow and temperature fields agreed well with measurements. Shamsoddini and Nezhad [96] presented a numerical simulation showing the effect of the inlet number on the tube performance and reported that the increase of the numbers of inlet nozzles led to an improvement of the tube performance. Experimental data was collected by Dincer et al. [97] in 2008 to train the ANN (artificial neural networks) model which was developed with the MATLAB code, and the prediction of the temperature difference from the model was compared with the experimental data.

The main limitation in applying computational analysis, is the uncertainty among the numerical simulations and the inconsistency observed between the simulation results and the experimental results. A partly modified standard $k-\varepsilon$ turbulence model revealed that there is an obvious energy separation effect in the vortex tube and the numerical solutions of the flow and temperature fields agreed well with the experimental data [98] (Figure 2.11). 


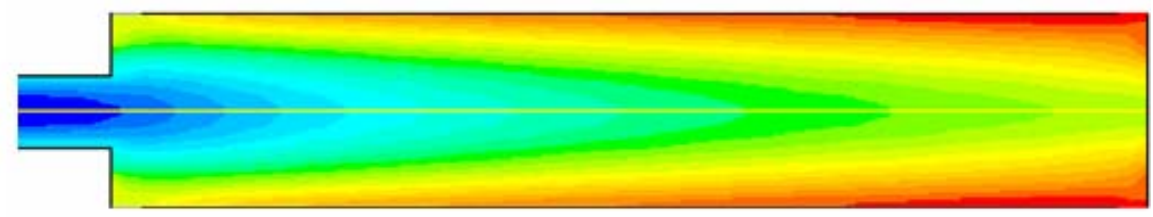

Figure 2.11. Total temperature distribution in the vortex tube [98]
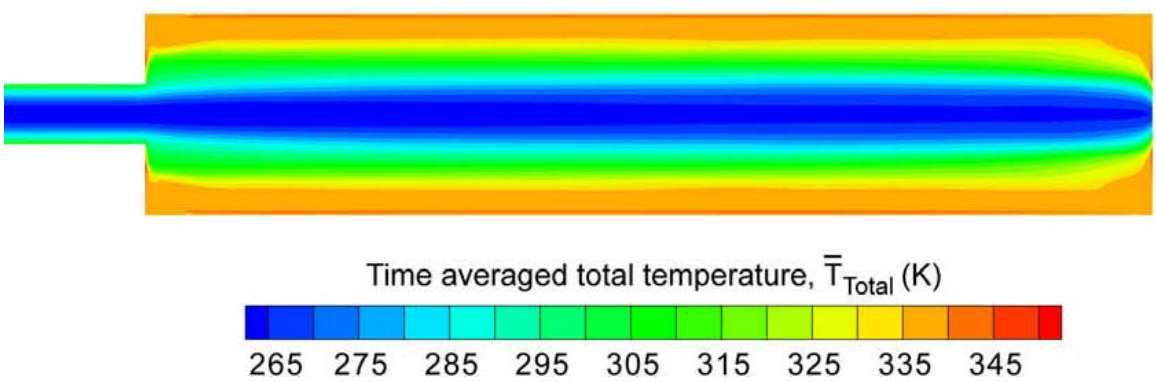

Figure 2.12. Total temperature distribution in the vortex tube [73]

However, Farouk \& Farouk in 2007 [72] and 2009 [73] applied a CFD-ACE+ code in the investigation using large eddy simulation. The velocity distribution, the temperature and the streamlines of the vortex flow were plotted. The temperature distribution predicted from the large eddy simulation worked better than the k- $\varepsilon$ model in prediction, as shown in Figure 2.12. The substantial difference between the temperature distribution predicted by the $\mathrm{k}-\varepsilon$ model and that predicted by the large eddy simulation can be seen in Figure 2.11 and Figure 2.12, which indicate the limitations of the numerical simulation of that research.

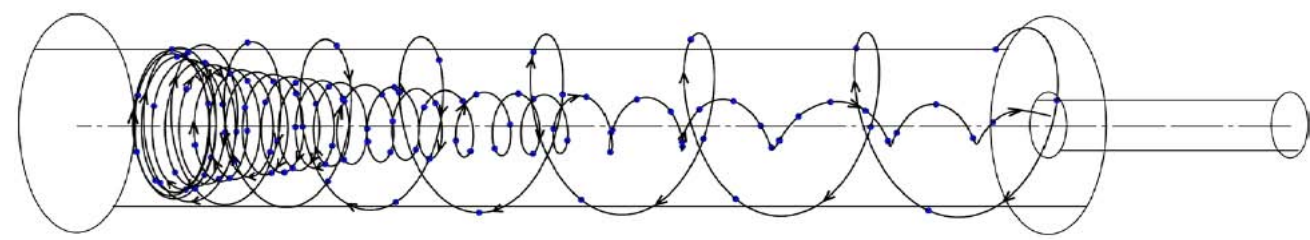

Figure 2.13. Flow trace of the inner layer in the vortex tube [72]

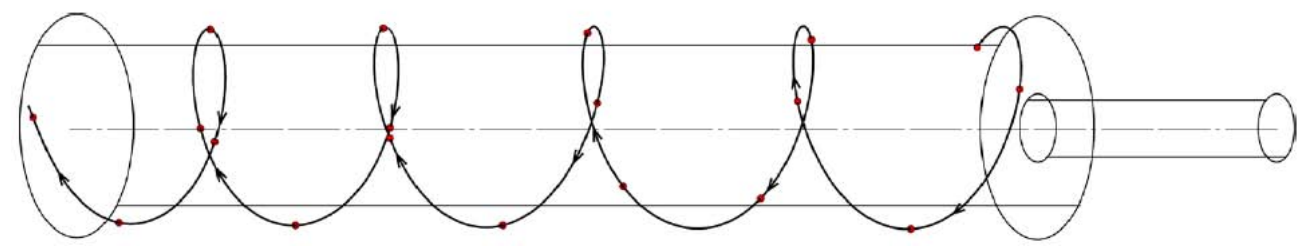

Figure 2.14. Flow trace of the outer layer in the vortex tube [72]

Simulation of the turbulent, compressible, high swirling flow was performed in 2009 by Secchiaroli et al. [99], using both RANS and LES techniques. Streamline 
and temperature gradient analysis were studied in that research, and the difference between the RANS and LES techniques was discussed. With the similar structure of the stream trace predicted by Farouk \& Farouk (Figure 2.13 and 2.14), the 3-D streamline in Figure 2.15 represents a different structure to the flow visualization result obtained by Aydin and Baki [69] (Figure 2.8). The flow behaviour shown in the numerical simulation indicates a different result with regard to the fact that the vortex angle increases in the middle of the tube after decreasing from the input point. The conflicting results between the flow structure in the flow visualization, and the numerical simulation indicates the necessity for further investigation.

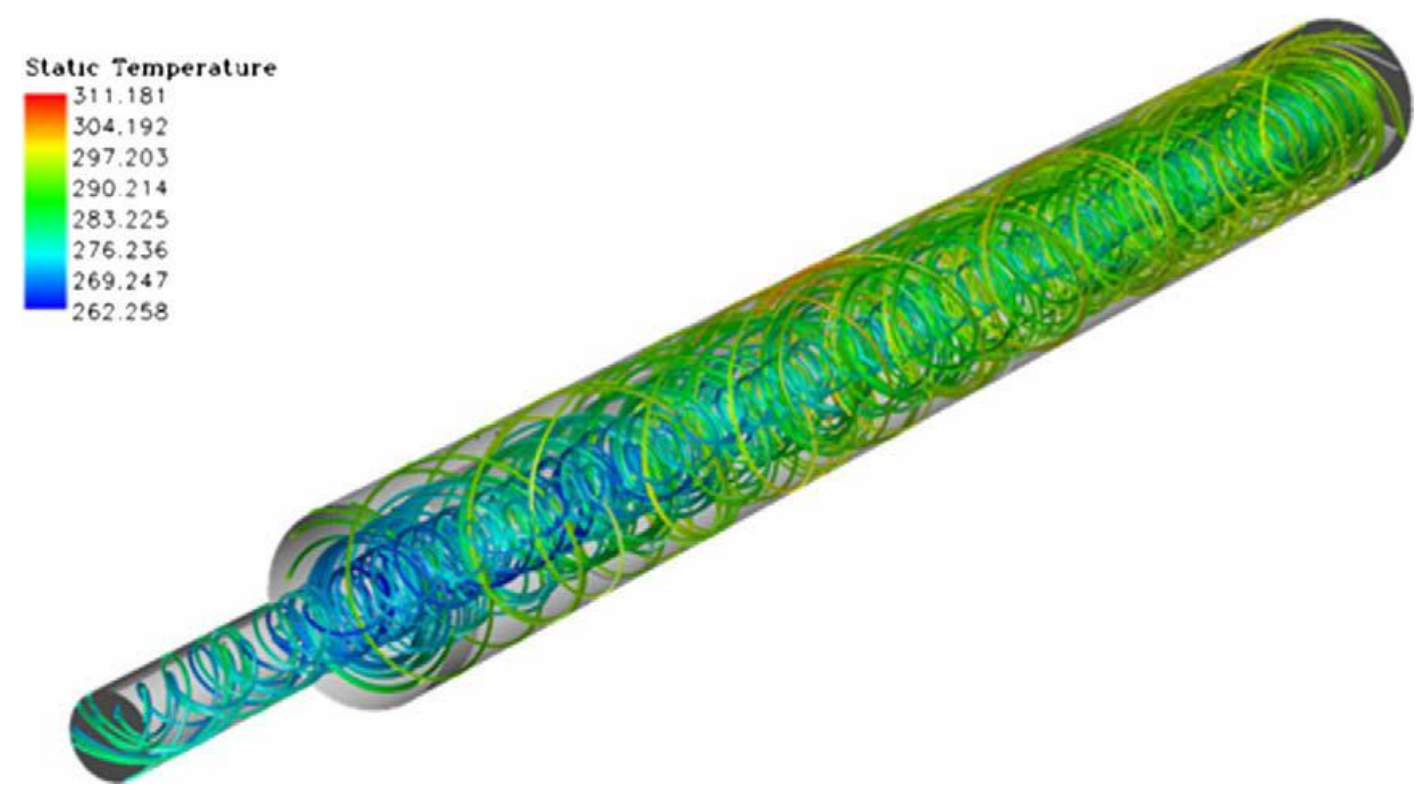

Figure 2.15. Streamlines patterns with temperature map [99]

Aljuwayhel et al. [87] reported on the energy separation and the flow phenomena in a counter-flow vortex tube using the commercial CFD code FLUENT and found that the RNG k-E model predicted the velocity and temperature variations better than the standard k- $\varepsilon$ model. This is in contrast to the results of Skye et al. [67], in which it is claimed that for determining the vortex tube's performance, the standard $\mathrm{k}-\varepsilon$ model performed better than the RNG $\mathrm{k}-\varepsilon$ model, despite using the same commercial CFD code. In 2010, Dutta et al. [100] compared different turbulence models in predicting the performance of the vortex tube and reported that the temperature separation predicted by the standard $\mathrm{k}-\varepsilon$ turbulence model was closer to the experimental results. Furthermore, in 2011, they simulated the detailed 
flow properties in the vortex tube using a real gas model and reported a small effect of the gas separation from the injected air [101].

As discussed above, the numerical simulation can provide detailed predictions of the flow field and flow properties inside a vortex tube. However, the disadvantages of the computational technology, which include different interpretations of the best turbulence model to use, conflicting simulation results and differences between the simulated and experimental performance, have resulted in inconsistencies in describing the flow field within the vortex tube and explaining the energy separation. Hence, the numerical simulation cannot provide a fully understood working principle of a vortex tube and further experimental and theoretical investigations are still required.

\subsection{Research objectives and methodology}

It is apparent from the above literature review that investigations of the vortex tube have been ongoing since its invention, and have focussed on establishing the mechanisms underlying its operation. Several explanations for the Ranque effect within a vortex tube have been proposed. However, none of these explanations can fully explain the whole process of separation and contradictory elements of these hypotheses have been reported. Further analysis of current hypotheses will be presented in chapter 3 .

To date, due to the complex flow conditions within the vortex tube, the physical process in the vortex tube remains unclear and an acceptable explanation has not been offered. This project aims to identify an acceptable explanation for the thermal separation occurring within the Ranque-Hilsch Vortex Tube based on a full understanding of the flow behaviour inside the vortex tube.

To achieve this outcome, the research objectives have been defined, and are:

- To understand the flow behaviour inside a vortex tube

- To develop and test an acceptable hypothesis for the energy separation in a vortex tube 
To outline the process to achieve these objectives, a short description of the research approach is discussed below.

As discussed above, due to the disadvantages of the computational simulation, high-fidelity experiments are operated in this investigation to provide detailed understanding of the flow field within a vortex tube. The internal flow field can be observed using appropriate visualization techniques. Water was selected in this research as the working fluid in a transparent vortex tube for the purpose of flow visualization, which enabled successful visualization by reducing the inlet velocity whilst maintaining the same Reynolds number at the inlet. Several visualization methods have been used to observe the flow behaviour inside the vortex tube. These are introduced in detailed in chapter 4, along with the observed results. Velocity profiles in the water-operated vortex tube were conducted based on the visualization results, and these were significantly correlated with the observed flow behaviour. Velocity profiles were also obtained in an air-operated vortex tube and these further confirmed the visualized flow structure and provided solid evidence for the hypothesized explanation. This part of the research is reported in chapter 5 .

The development of a novel hypothesis started from a critical analysis of the current explanations for the mechanism underlying temperature separation within the vortex tube. Evaluations of different factors in the separation were performed in the critical analysis, which were further used to develop the hypothesis. With the observed flow behaviour and measured flow parameters within the vortex tube, the hypothesis was finalized in chapter 6 . The validation of the proposed explanation consists of four parts, these being; confirmation of the flow structure; estimation of the temperature drop; exergy density analysis; and application of the proposed hypothesis in explaining the tube performance with varying parameters. 


\section{Reference}

[1] G.J. Ranque, Method and Apparatus for Obtaining from A Fluid under Pressure Two Outputs of Fluid at Different Temperatures, in, US, 1934, pp. p. 281.

[2] G.J. Ranque, Experiments on expansion in a vortex with simultaneous exhaust of hot air and cold air, J Phys Radium, 4 (1933) 112.

[3] R. Hilsch, The use of the expansion of gases in a centrifugal field as cooling process, Review of Scientific Instruments, 18 (1947) 108-113.

[4] R. Westley, A bibliography and survey of the vortex tube, College of Aeronautics. Cranfield note, UK, (1954).

[5] W. Curley, J.R. McGree, Bibliography of vortex tubes, Refrig Eng., 59 (1951) 191-193.

[6] L. Kalvinskas, Vortex Tubes (an Extension of Wesley's Bibliography), Jet Propulsion Laboratory, California Inst. of Technology Literature Search, (1956) 56 (Part 52).

[7] B.M. Dobratz, Vortex Tubes: a Bibliography, Lawrence Radiation Laboratory UCRL-7829, (1964).

[8] J.M. Nash, The Ranque-Hilsch vortex tube and its application to spacecraft environmental control systems, Dev Theor Appl Mech, 6 (1972) 6.

[9] Y. Soni, A Parametric Study of the Ranque-Hilsch Tube, in, University of Idaho Graduate School, USA, 1973.

[10] K.G. Hellyar, Gas Liquefaction using a Ranque-Hilsch Vortex Tube: Design Criteria and Bibliography, in, 1979.

[11] A.F. Gutsol, The Ranque effect, Physics-Uspekhi, 40 (1997) 639-658.

[12] A.I. Leont'ev, Gas-dynamic methods of temperature stratification, Fluid Dynamics, 37 (2002) 512-529.

[13] S. Eiamsa-ard, P. Promvonge, Review of Ranque-Hilsch effects in vortex tubes, Renewable and Sustainable Energy Reviews, 12 (2008) 1822-1842.

[14] M. Yilmaz, M. Kaya, S. Karagoz, S. Erdogan, A review on design criteria for vortex tubes, Heat and Mass Transfer/Waerme- und Stoffuebertragung, 45 (2009) 613-632.

[15] B.B. Parulekar, The short vortex tube, Journal of Refrigeration, 4 (1961) 74-80.

[16] H. Takahama, H. Yokosawa, Energy separation in vortex tubes with a divergent chamber, Journal of Heat Transfer, 103 (1981) 196-203. 
[17] I.L. Khodorkov, N.V. Poshernev, M.A. Zhidkov, Vortex tubes for gas heating, cooling, cleaning, drying, and separation, Khimicheskoe I Neftegazovoe Mashinostroenie, (2003) 24-27.

[18] I.L. Khodorkov, N.V. Poshernev, M.A. Zhidkov, The vortex tube - A universal device for heating, cooling, cleaning, and drying gases and separating gas mixtures, Chemical and Petroleum Engineering, 39 (2003) 409-415.

[19] I.L. Khodorkov, N.V. Poshernev, M.A. Zhidkov, Utilization of vortex tubes in processes of treating the gas mixtures, Gazovaya Promyshlennost, (2003) 82-84.

[20] N.V. Poshernev, I.L. Khodorkov, Experience from the operation of a conical vortex tube with natural gas, Chemical and Petroleum Engineering, 39 (2003) 602607.

[21] N.V. Poshernev, I.L. Khodorkov, Results of tests of conic vortex tube using the natural gas under external cooling, Khimicheskoe I Neftegazovoe Mashinostroenie, (2004) 18-20.

[22] N.V. Poshernev, I.L. Khodorkov, Natural-gas tests on a conical vortex tube (CVT) with external cooling, Chemical and Petroleum Engineering, 40 (2004) 212217.

[23] S.A. Piralishvili, V.M. Polyaev, Flow and thermodynamic characteristics of energy separation in a double-circuit vortex tube - An experimental investigation, Experimental Thermal and Fluid Science, 12 (1996) 399-410.

[24] S. Eiamsa-ard, K. Wongcharee, P. Promvonge, Experimental investigation on energy separation in a counter-flow Ranque-Hilsch vortex tube: Effect of cooling a hot tube, International Communications in Heat and Mass Transfer, 37 (2010) 156162.

[25] D.W. Guillaume, J.L. Jolly Iii, Demonstrating the achievement of lower temperatures with two-stage vortex tubes, Review of Scientific Instruments, 72 (2001) 3446-3448.

[26] K. Dincer, Experimental investigation of the effects of threefold type RanqueHilsch vortex tube and six cascade type Ranque-Hilsch vortex tube on the performance of counter flow Ranque-Hilsch vortex tubes, International Journal of Refrigeration, 34 (2011) 1366-1371.

[27] M.S. Valipour, N. Niazi, Experimental modeling of a curved Ranque-Hilsch vortex tube refrigerator, International Journal of Refrigeration, 34 (2011) 1109-1116.

[28] C.U. Linderstrøm-Lang, Gas separation in the Ranque-Hilsch vortex tube, International Journal of Heat and Mass Transfer, 7 (1964) 1195-1206.

[29] J. Marshall, Effect of operating conditions, physical size and fluid characteristics on the gas separation performance of a Linderstrom-Lang vortex tube, International Journal of Heat and Mass Transfer, 20 (1977) 227-231. 
[30] K. Volkan, Exergy analysis and performance of a counter flow Ranque-Hilsch vortex tube having various nozzle numbers at different inlet pressures of oxygen and air, International Journal of Refrigeration, 32 (2009) 1626-1633.

[31] K. Dincer, Y. Yilmaz, A. Berber, S. Baskaya, Experimental investigation of performance of hot cascade type Ranque-Hilsch vortex tube and exergy analysis, International Journal of Refrigeration, 34 (2011) 1117-1124.

[32] K. Polat, V. Kırmac1, Determining of gas type in counter flow vortex tube using pairwise fisher score attribute reduction method, International Journal of Refrigeration, 34 (2011) 1372-1386.

[33] K. Polat, V. Krmac, Application of the output dependent feature scaling in modeling and prediction of performance of counter flow vortex tube having various nozzles numbers at different inlet pressures of air, oxygen, nitrogen and argon, International Journal of Refrigeration, 34 (2011) 1387-1397.

[34] K.T. Raterman, M.G. Mc Kellar, T.D. Turner, A.K. Podgorney, D.E. Stacey, B. Stokes, J. Vranicar, A Vortex Contactor for Carbon Dioxide Separations, in: First National Conference on Carbon Sequestration, National Energy Technology Laboratory, U.S.A, Washington, DC, 2001.

[35] M.R. Kulkarni, C.R. Sardesai, Enrichment of methane concentration via separation of gases using vortex tubes, Journal of Energy Engineering, 128 (2002) 112.

[36] A.M. Crocker, G.L. Sutphin, D.V. Cassisi, C. Knowlen, R.F. Weimer, Investigation of enhanced vortex tube air separators for advanced space transportation, in: 40th AIAA/ASME/SAE/ASEE Joint Propulsion Conference and Exhibit, Fort Lauderdale, FL, 2004.

[37] H. Takahama, H. Kawamura, S. Kato, H. Yokosawa, Performance characteristics of energy separation in a steam-operated vortex tube, International Journal of Engineering Science, 17 (1979) 735-744.

[38] R.L. Collins, R.B. Lovelace, Experimental study of two-phase prophane expanded through the Ranque-Hilsch tube, Journal of Heat Transfer, 101 (1979) 300305.

[39] R.T. Balmer, Pressure-Driven Ranque-Hilsch Temperature Separation in Liquids, Journal of Fluids Engineering, Transactions of the ASME, 110 (1988) 161164.

[40] R. Shamsoddini, A. Faghih Khorasani, A new approach to study and optimize cooling performance of a Ranque-Hilsch vortex tube, International Journal of Refrigeration, 35 (2012) 2339-2348.

[41] R. Westley, Optimum design of a vortex tube for achieving large temperature drop ratios, College of Aeronautics, (1955).

[42] H. Takahama, Studies on vortex tubes, Bull. JSME, 8 (1965) 433-440. 
[43] H. Takahama, S. Kato, H. Kawamura, H. Yokosawa, Y. Kawahara, Study on a vortex tube (performance characteristics of a steam operated vortex tube), Trans. Japan soc. Mech. Engrs., 44 (1978).

[44] H. Takahama, K. Tanimoto, Study on vortex tubes (effect of the bend of a vortex chamber on energy separation), Bull JSME, 17 (1974) 740-747.

[45] H. Takahama, K. Tanimoto, Study on vortex tubes (effect of the bend of a vortex chamber on energy separation), Bull JSME, 17 (1974) 740-747.

[46] H. Takahama, H. Yokosawa, Study on vortex tubes. (Vortex energy separation in tube attached to cone), Trans. Japan soc. Mech. Engrs., 44 (1978) 988-996.

[47] H. Takahama, H. Yokosawa, T. Ohara, Study on vortex tubes (use of divergent tube to shorten length of main chamber), Trans. Japan soc. Mech. Engrs. SER. B., 46 (1980) 584-592.

[48] Y. Soni, A Parametric Study of the Ranque-Hilsch Tube, University of Idaho., 1973.

[49] Y.T. Wu, Y. Ding, Y.B. Ji, C.F. Ma, M.C. Ge, Modification and experimental research on vortex tube, International Journal of Refrigeration, 30 (2007) 1042-1049.

[50] S. Eiamsa-ard, Experimental investigation of energy separation in a counterflow Ranque-Hilsch vortex tube with multiple inlet snail entries, International Communications in Heat and Mass Transfer, 37 (2010) 637-643.

[51] K. Dincer, A. Avci, S. Baskaya, A. Berber, Experimental investigation and exergy analysis of the performance of a counter flow Ranque-Hilsch vortex tube with regard to nozzle cross-section areas, International Journal of Refrigeration, 33 (2010) 954-962.

[52] V. Krmac, O. Uluer, K. Dincer, An experimental investigation of performance and exergy analysis of a counterflow vortex tube having various nozzle numbers at different inlet pressures of air, oxygen, nitrogen, and argon, Journal of Heat Transfer, 132 (2010).

[53] N. Pourmahmoud, A. Hassanzadeh, O. Moutaby, Numerical analysis of the effect of helical nozzles gap on the cooling capacity of Ranque-Hilsch vortex tube, International Journal of Refrigeration, in press (2012).

[54] M. Arjomandi, X. Yunpeng, An investigation of the effect of the hot end plugs on the efficiency of the Ranque-Hilsch vortex tube, Journal of Engineering Science and Technology (JESTEC), 2 (2007) 211-217.

[55] C. Gao, Experimental study on the Ranque-Hilsch Vortex Tube, in, Technische Universiteit Eindhoven, 2005.

[56] L.M. Dyskin, Characteristics of a vortex tube with detwisting of cold flow, Journal of Engineering Physics and Thermophysics, 57 (1989) 756-758. 
[57] A.I. Gulyaev, Investigation of conical vortex tubes, Journal of Engineering Physics and Thermophysics, 10 (1966) 193-195.

[58] K. Chang, Q. Li, G. Zhou, Experimental investigation of vortex tube refrigerator with a divergent hot tube, International Journal of Refrigeration, 34 (2011) 322-327.

[59] Y. Xue, M. Arjomandi, The effect of vortex angle on the efficiency of the Ranque-Hilsch vortex tube, Experimental Thermal and Fluid Science, 33 (2008) 5457.

[60] O. Aydin, B. Markal, M. Avci, A new vortex generator geometry for a counterflow Ranque-Hilsch vortex tube, Applied Thermal Engineering, 30 (2010) 25052511.

[61] B. Markal, O. AydIn, M. AvcI, An experimental study on the effect of the valve angle of counter-flow Ranque-Hilsch vortex tubes on thermal energy separation, Experimental Thermal and Fluid Science, 34 (2010) 966-971.

[62] B. Markal, O. Aydin, M. Avci, Exergy analysis of a counter-flow RanqueHilsch vortex tube having different helical vortex generators, International Journal of Exergy, 10 (2012) 228-238.

[63] A.F. Hamoudi, A. Fartaj, G.W. Rankin, Performance characteristics of a microscale ranque-hilsch vortex tube, Journal of Fluids Engineering, Transactions of the ASME, 130 (2008) 1012061-1012068.

[64] J.R. Simões-Moreira, An air-standard cycle and a thermodynamic perspective on operational limits of Ranque-Hilsh or vortex tubes, International Journal of Refrigeration, 33 (2010) 765-773.

[65] U. Behera, P.J. Paul, S. Kasthurirengan, R. Karunanithi, S.N. Ram, K. Dinesh, S. Jacob, CFD analysis and experimental investigations towards optimizing the parameters of Ranque-Hilsch vortex tube, International Journal of Heat and Mass Transfer, 48 (2005) 1961-1973.

[66] U. Behera, P.J. Paul, K. Dinesh, S. Jacob, Numerical investigations on flow behaviour and energy separation in Ranque-Hilsch vortex tube, International Journal of Heat and Mass Transfer, 51 (2008) 6077-6089.

[67] H.M. Skye, G.F. Nellis, S.A. Klein, Comparison of CFD analysis to empirical data in a commercial vortex tube, International Journal of Refrigeration, 29 (2006) 71-80.

[68] W. Fröhlingsdorf, H. Unger, Numerical investigations of the compressible flow and the energy separation in the Ranque-Hilsch vortex tube, International Journal of Heat and Mass Transfer, 42 (1999) 415-422.

[69] O. Aydin, M. Baki, An experimental study on the design parameters of a counterflow vortex tube, Energy, 31 (2006) 2427-2436. 
[70] B. Markal, O. Aydın, M. Avc1, An experimental study on the effect of the valve angle of counter-flow Ranque-Hilsch vortex tubes on thermal energy separation, Experimental Thermal and Fluid Science, 34 (2010) 966-971.

[71] V.A. Arbuzov, Y.N. Dubnishchev, A.V. Lebedev, M.K. Pravdina, N.I. Yavorski $\check{1}$, Observation of large-scale hydrodynamic structures in a vortex tube and the Ranque effect, Technical Physics Letters, 23 (1997) 938-940.

[72] T. Farouk, B. Farouk, Large eddy simulations of the flow field and temperature separation in the Ranque-Hilsch vortex tube, International Journal of Heat and Mass Transfer, 50 (2007) 4724-4735.

[73] T. Farouk, B. Farouk, A. Gutsol, Simulation of gas species and temperature separation in the counter-flow Ranque-Hilsch vortex tube using the large eddy simulation technique, International Journal of Heat and Mass Transfer, 52 (2009) 3320-3333.

[74] M.H. Saidi, M.R. Allaf Yazdi, Exergy model of a vortex tube system with experimental results, Energy, 24 (1999) 625-632.

[75] M.H. Saidi, M.S. Valipour, Experimental modeling of vortex tube refrigerator, Applied Thermal Engineering, 23 (2003) 1971-1980.

[76] K. Dincer, S. Baskaya, B.Z. Uysal, Experimental investigation of the effects of length to diameter ratio and nozzle number on the performance of counter flow Ranque-Hilsch vortex tubes, Heat and Mass Transfer/Waerme- und Stoffuebertragung, 44 (2008) 367-373.

[77] K. Dincer, S. Baskaya, B.Z. Uysal, I. Ucgul, Experimental investigation of the performance of a Ranque-Hilsch vortex tube with regard to a plug located at the hot outlet, International Journal of Refrigeration, 32 (2009) 87-94.

[78] S.U. Nimbalkar, M.R. Muller, An experimental investigation of the optimum geometry for the cold end orifice of a vortex tube, Applied Thermal Engineering, 29 (2009) 509-514.

[79] P.K. Singh, R.G. Tathgir, D. Gangacharyulu, G.S. Grewal, An experimental performance evaluation of vortex tube, Journal of the Institution of Engineers (India): Mechanical Engineering Division, 84 (2003) 149-153.

[80] S. Eiamsa-Ard, P. Promvonge, Simulation of turbulent flow and temperature separation in a uni-flow vortex tube, Songklanakarin Journal of Science and Technology, 29 (2007) 459-475.

[81] S. Eiamsa-ard, P. Promvonge, Numerical investigation of the thermal separation in a Ranque-Hilsch vortex tube, International Journal of Heat and Mass Transfer, 50 (2007) 821-832. 
[82] S. Eiamsa-Ard, P. Promvonge, Numerical simulation of flow field and temperature separation in a vortex tube, International Communications in Heat and Mass Transfer, 35 (2008) 937-947.

[83] O.V. Kazantseva, S.A. Piralishvili, A.A. Fuzeeva, Numerical simulation of swirling flows in vortex tubes, High Temperature, 43 (2005) 608-613.

[84] B. Ahlborn, S. Groves, Secondary flow in a vortex tube, Fluid Dynamics Research, 21 (1997) 73-86.

[85] B.K. Ahlborn, J.M. Gordon, The vortex tube as a classic thermodynamic refrigeration cycle, Journal of Applied Physics, 88 (2000) 3645-3653.

[86] B.K. Ahlborn, J.U. Keller, E. Rebhan, The Heat Pump in a Vortex Tube, Journal of Non-Equilibrium Thermodynamics, 23 (1998) 159-165.

[87] N.F. Aljuwayhel, G.F. Nellis, S.A. Klein, Parametric and internal study of the vortex tube using a CFD model, International Journal of Refrigeration, 28 (2005) 442-450.

[88] S.Y. Im, S.S. Yu, Effects of geometric parameters on the separated air flow temperature of a vortex tube for design optimization, Energy, 37 (2012) 154-160.

[89] M.O. Hamdan, A. Alawar, E. Elnajjar, W. Siddique, Experimental analysis on vortex tube energy separation performance, Heat and Mass Transfer/Waerme- und Stoffuebertragung, 47 (2011) 1637-1642.

[90] J.E. Lay, An experimental and analytical study of vortex flow temperature separation by superposition of spiral and axial flows: Part II, Trans ASME J Heat Transfer, 81 (1959) 202-212.

[91] C. Sohn, C.-S. Kim, U.-H. Jung, B. Gowda, Experimental and Numerical Studies in a Vortex Tube, Journal of Mechanical Science and Technology, 20 (2006) 418-425.

[92] J. R.MacGee, Fluid Action in the Vortex Tube, J. ASRE Refrigerating Engng, 58 (1950) 974-975.

[93] M. Sibulkin, Unsteady viscous circular flow - Application to the Ranque Hilsch vortex tube, J. Fluid Mech., 12 (1962) 269-293.

[94] J.L. Smith, An analysis of the vortex flow in the cyclone separator, Journal of Basic Engineering-Transactions of the ASME, (1962) 609-618.

[95] J.L. Smith, An experimental study of the vortex in the cyclone separator, Journal of Basic Engineering-Transactions of the ASME, 84 (1962) 602-608.

[96] R. Shamsoddini, A.H. Nezhad, Numerical analysis of the effects of nozzles number on the flow and power of cooling of a vortex tube, International Journal of Refrigeration, 33 (2010) 774-782. 
[97] K. Dincer, Ş. Taşdemir, Ş. Başkaya, B.Z. Uysal, Modeling of the effects of plug tip angle on the performance of counter-flow Ranque-Hilsch vortex tubes using artificial neural networks, Journal of Thermal Science and Technology, 28 (2008) 17.

[98] J.Y. Liu, M.Q. Gong, Y. Zhang, H. Hong, J.F. Wu, Numerical research on a special fluid phenomenon: Ranque-hilsch effect, Modern Physics Letters B, 19 (2005) 1723-1726.

[99] A. Secchiaroli, R. Ricci, S. Montelpare, V. D'Alessandro, Numerical simulation of turbulent flow in a Ranque-Hilsch vortex tube, International Journal of Heat and Mass Transfer, 52 (2009) 5496-5511.

[100] T. Dutta, K.P. Sinhamahapatra, S.S. Bandyopdhyay, Comparison of different turbulence models in predicting the temperature separation in a Ranque-Hilsch vortex tube, International Journal of Refrigeration, 33 (2010) 783-792.

[101] T. Dutta, K.P. Sinhamahapatra, S.S. Bandyopadhyay, Numerical investigation of gas species and energy separation in the Ranque-Hilsch vortex tube using real gas model, International Journal of Refrigeration, 34 (2011) 2118-2128. 


\section{Chapter 3}

\section{Analysis of the available hypothesises}

\subsection{Introduction}

A critical review of current explanations for the temperature separation in a vortex tube is presented in this chapter. The manuscript reports critical analysis of current explanations, including pressure gradient, viscosity, turbulence, static temperature, secondary circulation and acoustic streaming. Experimental results, numerical simulations and theoretical calculations are used to perform the analysis, by which the effects of each hypothesis are evaluated.

It was concluded in the review that none of the explanations could be used to explain the whole working process within a vortex tube and even opposite statements about the flow behavior were reported. Based on the critical analysis, a novel hypothesis was proposed, which contained the evaluation of expansion and the consideration of the wall temperature gradient. It is proposed that partial stagnation and mixture due to the structure of multi-circulation in the rear part of the tube, and the pressure gradient near the injection port are the primary factors for the generation of the two streams at different temperatures in a vortex tube. The validation of the hypothesised flow behaviour is presented in the following chapters. 


\title{
3.2. A critical review of temperature separation in a vortex tube
}

\author{
Experimental Thermal and Fluid Science, 34 (2010) 1367-1374
}

\section{Statement of authorship}

Yunpeng Xue (candidate)

Performed analysis and wrote manuscript.

Signed:

Date

Dr. Maziar Arjomandi (Principal Supervisor)

Contributed in academic discussion and manuscript review

Signed:

Date

Associate Professor Richard Kelso (Co-supervisor)

Contributed in academic discussion and manuscript review

Signed:

Date 
Y. Xue, M. Arjomandi and R. Kelso (2010) A critical review of temperature separation in a vortex tube.

Experimental Thermal and Fluid Science, v. 34(8), pp. 1367-1374, November 2010

NOTE: This publication is included on pages $41-48$ in the print copy of the thesis held in the University of Adelaide Library.

It is also available online to authorised users at:

http://dx.doi.org/10.1016/j.expthermflusci.2010.06.010 


\section{Chapter 4}

\section{Visualization of the internal flow behaviour}

\subsection{Introduction}

This chapter discusses the flow behaviour observed inside a counter-flow vortex tube via flow visualization. A good understanding of the flow behaviour inside the vortex tube is an essential requirement in the process of identifying the dominant mechanisms for the temperature separation. The results of flow visualization assist in a better understanding of the flow behaviour inside the vortex tube and provide direct evidence for the proposed hypothesis.

In order to observe the flow behavior inside a transparent vortex tube, water was used as the working medium, which ensures the similar Reynolds number at the inlet when low velocity was set. Moreover, as reported in the article, the pressure gradient within a vortex tube enables the prediction of the flow behavior in the hot region of the vortex tube using the visualization results conducted from a wateroperated vortex tube. Different visualizing materials were injected into the vortex tube, by which the flow structures in the cold region and multi-circulation region were observed. The axial and swirl velocities were also measured to understand the flow behaviour and allow calculation of the volume flow rate along the vortex tube.

The flow structure inside a water-operated vortex tube is summarised based on the visualization results, which agree with the hypothesised flow structure, and hence supports the proposed explanation. To further confirm the flow behaviour within the vortex, an air-operated vortex tube was designed, in which a temperature difference between the two exhausted streams was generated. The results of measurements of the velocity profiles and flow properties within the air-operated vortex tube, are reported in the following chapter which support the proposed flow behaviour and hypothesis. 


\subsection{Visualization of flow structure in a vortex tube}

Experimental Thermal and Fluid Science 35(8) (2011) 1514-1521

\section{Statement of authorship}

Yunpeng Xue (Candidate)

Performed experimental work, analysed data and wrote manuscript.

Signed:

Date

Dr. Maziar Arjomandi (Principal Supervisor)

Supervised the research and contributed in academic discussion and manuscript review

Signed:

Date

Associate Professor Richard Kelso (Co-supervisor)

Supervised the research and contributed in academic discussion and manuscript review

Signed:

Date 
Y. Xue, M. Arjomandi and R. Kelso (2011) Visualization of flow structure in a vortex tube.

Experimental Thermal and Fluid Science, v. 35(8), pp. 1514-1521, November 2011

NOTE: This publication is included on pages $51-58$ in the print copy of the thesis held in the University of Adelaide Library.

It is also available online to authorised users at:

http://dx.doi.org/10.1016/j.expthermflusci.2011.07.001 


\section{Chapter 5}

\section{Flow behaviour inside an air-operated vortex tube}

\subsection{Introduction}

This chapter reports on the experimental investigations of the flow structure inside an air-operated vortex tube, and the results comprise the material presented in two journal papers.

The first paper, entitled "Experimental study of the flow structure in a counter flow Ranque-Hilsch vortex tube", shows the optimization of a vortex tube with variable geometrical parameters. Three-dimensional velocity distributions inside a counter-flow vortex tube are presented in detail in this paper. The results of measurements in the optimized tube show a transition from a forced vortex at the cold end of the tube to an irrotational vortex at the hot end, which is supported by the static pressure gradient.

The second paper, entitled "Experimental study of the thermal separation in a vortex tube", reports further analysis of the flow properties inside the tube, including turbulence intensity and velocity, temperature and pressure profiles. The proposed hypothesis is well supported by the reported flow structure. An exergy analysis based on the experimental data indicates that there is negligible energy transferred between different layers of the internal flow and provides solid evidence for the stated hypothesis.

With the further confirmation of the flow behavior and support for the hypothesis, the finalized explanation is introduced in chapter 6 , together with further evidence for the flow structure, exergy analysis, estimation of temperature drop, and discussions of the geometric effects on the tube performance. 


\subsection{Experimental study of the flow structure in a counter flow vortex tube}

International Journal of Heat and Mass Transfer, 55(21-22) (2012) 5853-5860

\section{Statement of authorship}

Yunpeng Xue (candidate)

Performed experimental work, analysed data and wrote manuscript.

Signed:

Date

Dr. Maziar Arjomandi (Principal Supervisor)

Supervised the research and contributed in academic discussion and manuscript review

Signed:

Date

Associate Professor Richard Kelso (Co-supervisor)

Supervised the research and contributed in academic discussion and manuscript review

Signed:

Date 
Y. Xue, M. Arjomandi and R. Kelso (2012) Experimental study of the flow structure in a counter flow vortex tube.

International Journal of Heat and Mass Transfer, v. 55(21-22), pp. 5853-5860, October 2012

NOTE: This publication is included on pages 61 - 68 in the print copy of the thesis held in the University of Adelaide Library.

It is also available online to authorised users at:

http://dx.doi.org/10.1016/j.ijheatmasstransfer.2012.05.081 


\subsection{Experimental study of the thermal separation in a vortex tube}

Experimental Thermal and Fluid Science, 46 (2013), 175-182

\section{Statement of authorship}

Yunpeng Xue (candidate)

Performed experimental work, analysed data and wrote manuscript.

Signed:

Date

Dr. Maziar Arjomandi (Principal Supervisor)

Supervised the research and contributed in academic discussion and manuscript review

Signed:

Date

Associate Professor Richard Kelso (Co-supervisor)

Supervised the research and contributed in academic discussion and manuscript review

Signed:

Date 
Y. Xue, M. Arjomandi and R. Kelso (2013) Experimental study of the thermal separation in a vortex tube.

Experimental Thermal and Fluid Science, v. 46, pp. 175-182, February 2013

NOTE: This publication is included on pages $71-78$ in the print copy of the thesis held in the University of Adelaide Library.

It is also available online to authorised users at:

http://dx.doi.org/10.1016/j.expthermflusci.2012.12.009 


\section{Chapter 6}

\section{Analytical assessment of the proposed hypothesis}

\subsection{Introduction}

Based on the research outcomes, the finalized hypothesis for the Ranque effect in a vortex tube is presented in this chapter, as well as further validations from the theoretical analysis. Two papers are included in this chapter.

The first paper, entitled "Energy analysis within a vortex tube", reports the finalized hypothesis for the energy separation in a vortex tube and further analysis of the exergy density along the vortex tube. The exergy density gradient is calculated based on the collected data from both this and other experimental investigations, which provides conclusive evidence for the explanation offered in this study. The good agreement between the trends in the exergy density gradient strengthens the acceptance of the proposed explanation.

The second paper, entitled "The working principle of a vortex tube", concludes the experimental support for the proposed explanation generated in this research. The acceptance, accuracy and reliability of the proposed hypothesis are indicated by the estimation of the temperature drop based on the assumption of a forced vortex model at the cold end. The working principle of a vortex tube is fully discussed at variable cold mass flow ratios, which agree well with the experimental results. Explanations of the geometrical effects on the tube performance based on the explanation are in accordance with the experimental findings, hence further indicating the validity of the explanation for the temperature separation in a vortex tube. 


\subsection{Energy analysis within a vortex tube}

Experimental Thermal and Fluid Science (Under review)

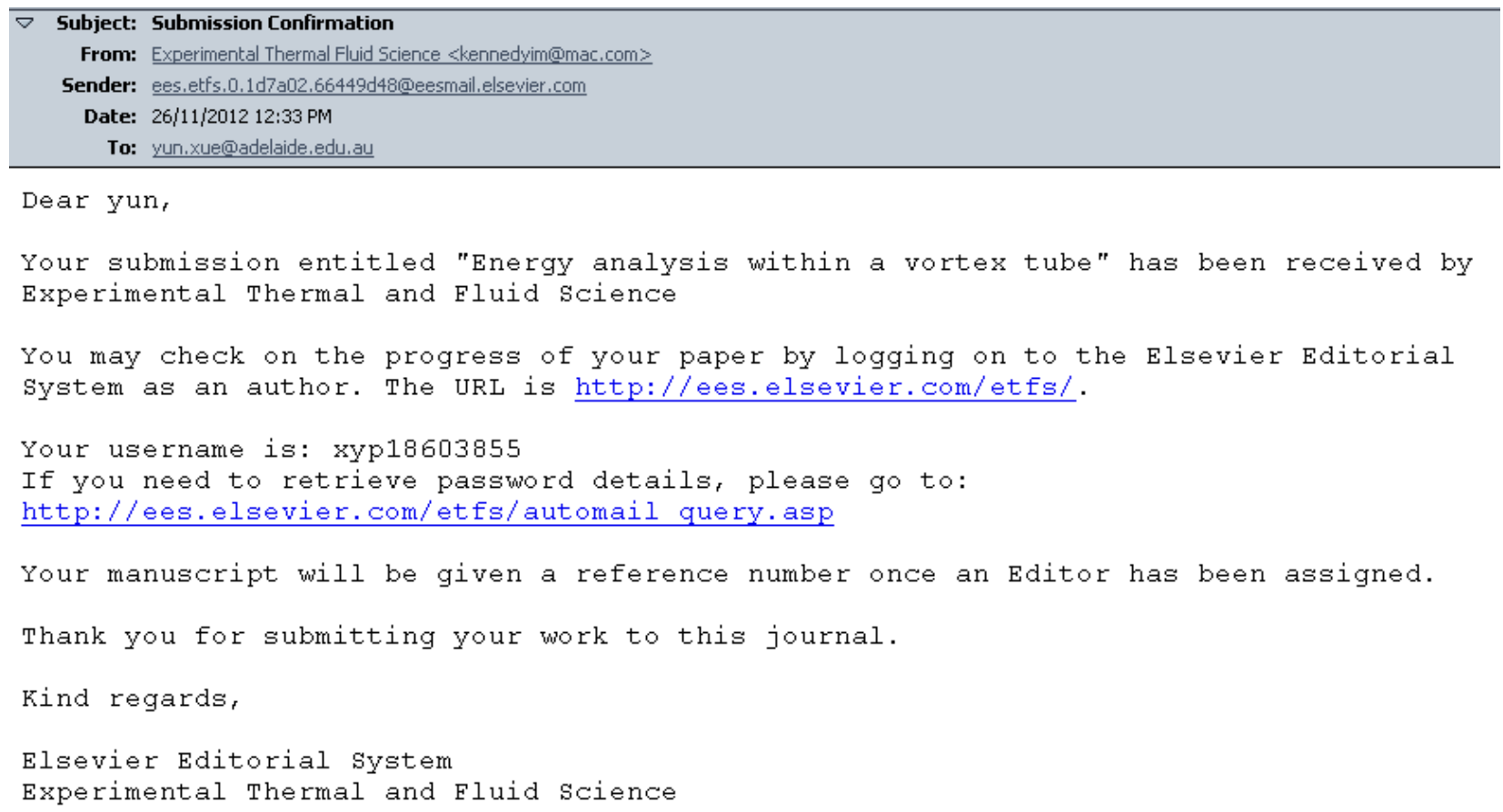




\section{Statement of authorship}

\section{Energy analysis within a vortex tube}

Yunpeng Xue (candidate)

Performed experimental work, analysed data and wrote manuscript.

Signed: $\quad$ Date

Dr. Maziar Arjomandi (Principal Supervisor)

Supervised the research and contributed in academic discussion and manuscript review

Signed: _ Date

Associate Professor Richard Kelso (Co-supervisor)

Supervised the research and contributed in academic discussion and manuscript review

Signed:

Date 


\title{
Energy analysis within a vortex tube
}

\author{
Yunpeng Xue, Maziar Arjomandi and Richard Kelso
}

School of Mechanical Engineering, the University of Adelaide

South Australia 5005, Australia

The generation of separated cold and hot streams from a single injection in a vortex tube is known as the Ranque effect. Since its invention, several explanations concerning the phenomenon of thermal separation in a vortex tube have been proposed, however there has not been a consensus, due to the complexity of the physical process inside the tube.

This paper proposes an explanation for the temperature separation in a vortex tube based on an experimental study focusing on the flow structure and energy analysis inside the tube. Using the measured flow properties inside the tube, the exergy density distribution along the vortex tube was calculated, from which the reasons for the temperature separation were identified. The good agreement of the exergy density analysis with findings from other experimental work supports the validity of the proposed hypothesis. Based on the forced vortex assumption, the minimum temperature in a vortex tube was calculated, which correlated well with the collected experimental results.

Keywords: Ranque effect, Ranque-Hilsch vortex tube, energy separation, exergy density, thermal separation, vortex flow

\begin{tabular}{|l|l|}
\hline \multicolumn{2}{|l|}{ Nomenclature } \\
\hline$\theta$ & Exergy density ratio \\
\hline$\rho$ & density $\left(\mathrm{kg} / \mathrm{m}^{3}\right)$ \\
\hline$C_{p}$ & Specific heat at constant pressure $(\mathrm{kJ} / \mathrm{kg} \mathrm{K})$ \\
\hline$D$ & Diameter of the vortex tube $(\mathrm{mm})$ \\
\hline$d h$ & Change of enthalpy $(\mathrm{J})$ \\
\hline$d p$ & Pressure change $(\mathrm{Pa})$ \\
\hline$d s$ & Entropy change $(\mathrm{J})$ \\
\hline$E$ & Energy of the control volume $(\mathrm{J})$ \\
\hline ex & Exergy density $\left(\mathrm{J} / \mathrm{m}^{3}\right)$ \\
\hline
\end{tabular}




\begin{tabular}{|c|c|}
\hline Ex & Exergy of the control volume $(\mathrm{J})$ \\
\hline$g$ & Gravitational acceleration \\
\hline$H$ & Enthalpy of the control volume $(\mathrm{J})$ \\
\hline$I_{u v w}$ & Overall turbulence intensity \\
\hline 1 & Axial location $(\mathrm{mm})$ \\
\hline$L$ & Effective length of the vortex tube from inlet to the plug $(\mathrm{mm})$ \\
\hline$m$ & Mass (kg) \\
\hline$P$ & pressure $(\mathrm{Pa})$ \\
\hline$\delta Q$ & Infinitesimal transfer of heat to the control volume $(\mathrm{J})$ \\
\hline$r$ & Radial location (mm) \\
\hline$R$ & Radius of the vortex tube $(\mathrm{mm})$ \\
\hline $\boldsymbol{R}$ & Universal gas constant \\
\hline$S$ & Entropy of the system $(\mathrm{J})$ \\
\hline$\Delta T$ & Temperature difference $(K)$ \\
\hline$T$ & Temperature $(K)$ \\
\hline$v$ & velocity $(\mathrm{m} / \mathrm{s})$ \\
\hline $\bar{v}$ & Time-averaged overall velocity $(\mathrm{m} / \mathrm{s})$ \\
\hline$v^{\prime}$ & Time-varying velocity fluctuating component $(\mathrm{m} / \mathrm{s})$ \\
\hline$V$ & Volume $\left(m^{3}\right)$ \\
\hline$z$ & Net height $(m)$ \\
\hline \multicolumn{2}{|l|}{ Subscripts } \\
\hline$g$ & Gravitational \\
\hline$i$ & Instantaneous conditions \\
\hline in & Input parameter \\
\hline$k$ & Kinetic energy \\
\hline$k$, Mean & Average kinetic energy \\
\hline$k$, Turbulence & Kinetic energy of turbulent component \\
\hline 0 & Reference conditions \\
\hline$t$ & Total \\
\hline
\end{tabular}




\section{Introduction}

Invented by Ranque in 1933, the vortex tube has been used in many industrial applications, due to its simplicity and ability to generate separated cold and hot streams from a single injection. Figure 1 shows the structure of a counter-flow vortex tube, which consists of a straight tube with a port for tangential injection and exits at each of the tube ends. A control plug, with a smaller diameter than the tube diameter, is positioned inside the tube away from the injection end to allow the gas to escape from the small gap between the control plug and the tube wall. The cold exit is located in the central part of the tube end closest to the injection, while the hot exit is at the far end of the tube and comprises the gap between the central plug and the tube wall. When compressed air is injected into the tube from the tangential inlet, it forms a highly vortical flow as it moves to the other end of the tube. The peripheral part of the airflow escapes from the hot exit at a temperature higher than the inlet temperature and the core part of the airflow, which is turned back by the plug in a counter-flow vortex tube, escapes from the cold nozzle at a temperature lower than the temperature of the fed air. Thus, with a single injection, the injected flow is divided into two streams with different temperatures, which is known as the phenomenon of temperature separation or the Ranque effect. As demonstrated in Figure 1, a vortex tube does not have any internal or moving parts. Hence, the temperature separation effect in a vortex tube can be explained solely using fluid dynamical theories.

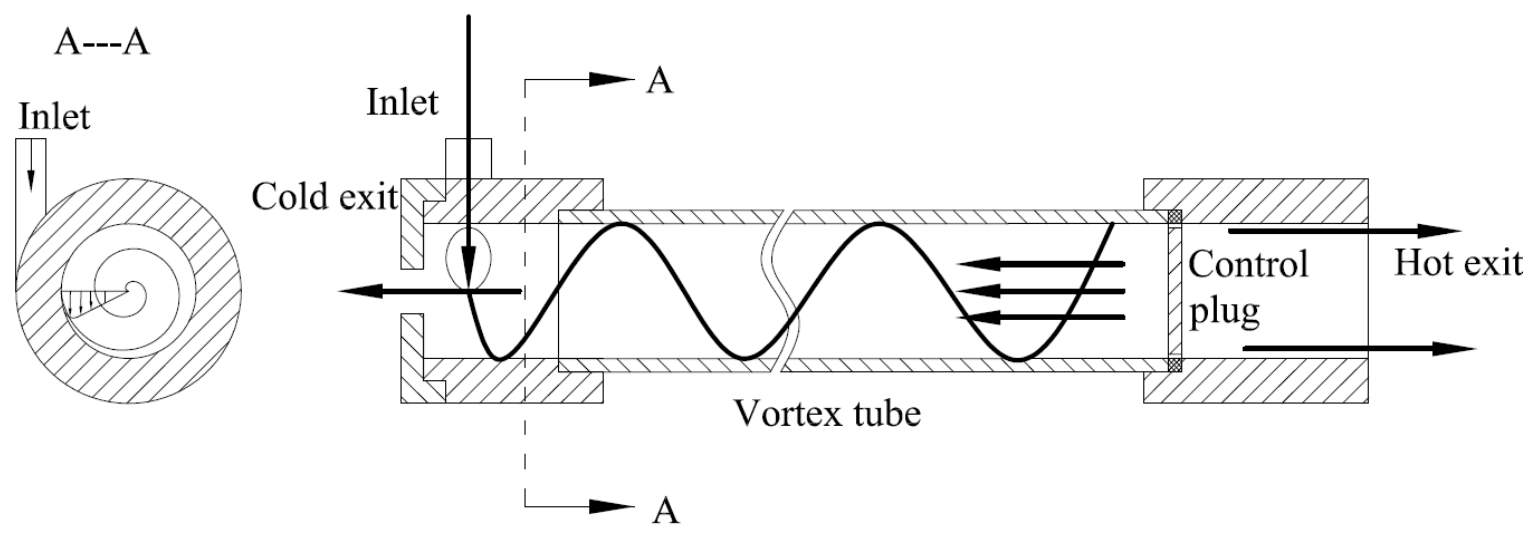

Figure.1.Schematic of a counter-flow vortex tube 
Xue et al. summarised [1] different hypotheses describing the main process of thermal separation in a vortex tube based on experimental, theoretical and numerical work. The critical analysis of the presented theories reveals that a well-accepted explanation for the temperature separation in a vortex tube has not been proposed. To identify the dominant factors of the thermal separation in a vortex tube, an understanding of the physical processes inside the tube is required. In another work, Xue et al. [2] conducted a qualitative analysis of the flow behaviour in a vortex tube using visualization of the flow inside a transparent tube. They showed that in an operating vortex tube, a portion of the central flow moved outwards and returned to the hot end in a flow pattern, termed multi-circulation. Hence, they hypothesized that the generation of two flow streams with different temperatures is due to the expansion of the injected gas near the inlet and stagnation of the multi-circulating flow near the hot end.

This paper proposes a new explanation for the temperature separation in a vortex tube based on an analysis of the internal exergy density distribution along the vortex tube. To identify the governing factors affecting the temperature separation, clarification of the energy transfer between different layers of the internal flow is required. According to detailed measurement results, the exergy density gradient inside the vortex tube can be used to show and verify the energy transfer between flow layers. It is found that in the front part of the vortex tube, i.e., also known as the cold part, the exergy density in the central region drops dramatically. This is associated with the process of injecting gas to the central region of the tube and indicates the effect of a sudden expansion. The slightly changed exergy density of the peripheral flow in the rear part of the vortex tube, i.e., also known as the hot part, suggests that there is negligible energy transferred outwards and that the temperature rise in the vortex tube is mainly caused by the partial stagnation and mixture due to the multi-circulation in the rear part of the tube. This is in contrast to the effects of secondary circulation reported by Ahlborn and Groves [3, 4]

\section{Experimental apparatus}


To investigate the exergy density, the flow properties inside a large-scale vortex tube with a length of $2000 \mathrm{~mm}$ and a diameter of $60 \mathrm{~mm}$ were measured (Figure 2). The large diameter of the vortex tube is selected to ensure accurate measurement and to minimise the turbulence induced by the intrusive equipment. A round nozzle with a diameter of $6 \mathrm{~mm}$, a cold exit with a diameter of $14 \mathrm{~mm}$ and a hot exit of $1 \mathrm{~mm}$ gap, formed by inserting a $58 \mathrm{~mm}$ plug into the $60 \mathrm{~mm}$ tube, were chosen based on the optimization of the tube performance as reported in [5].

A Cobra probe was used for the measurement of the flow properties inside the vortex tube, including 3-D velocity, static pressure and turbulence intensity of the flow. Data from the Cobra probe was logged and processed using the Turbulent Flow Instrumentation control software. The cobra probe was inserted into the vortex tube through the drilled holes along the tube and the small dimension of the probe head ensured minimum disturbance to the internal flow.

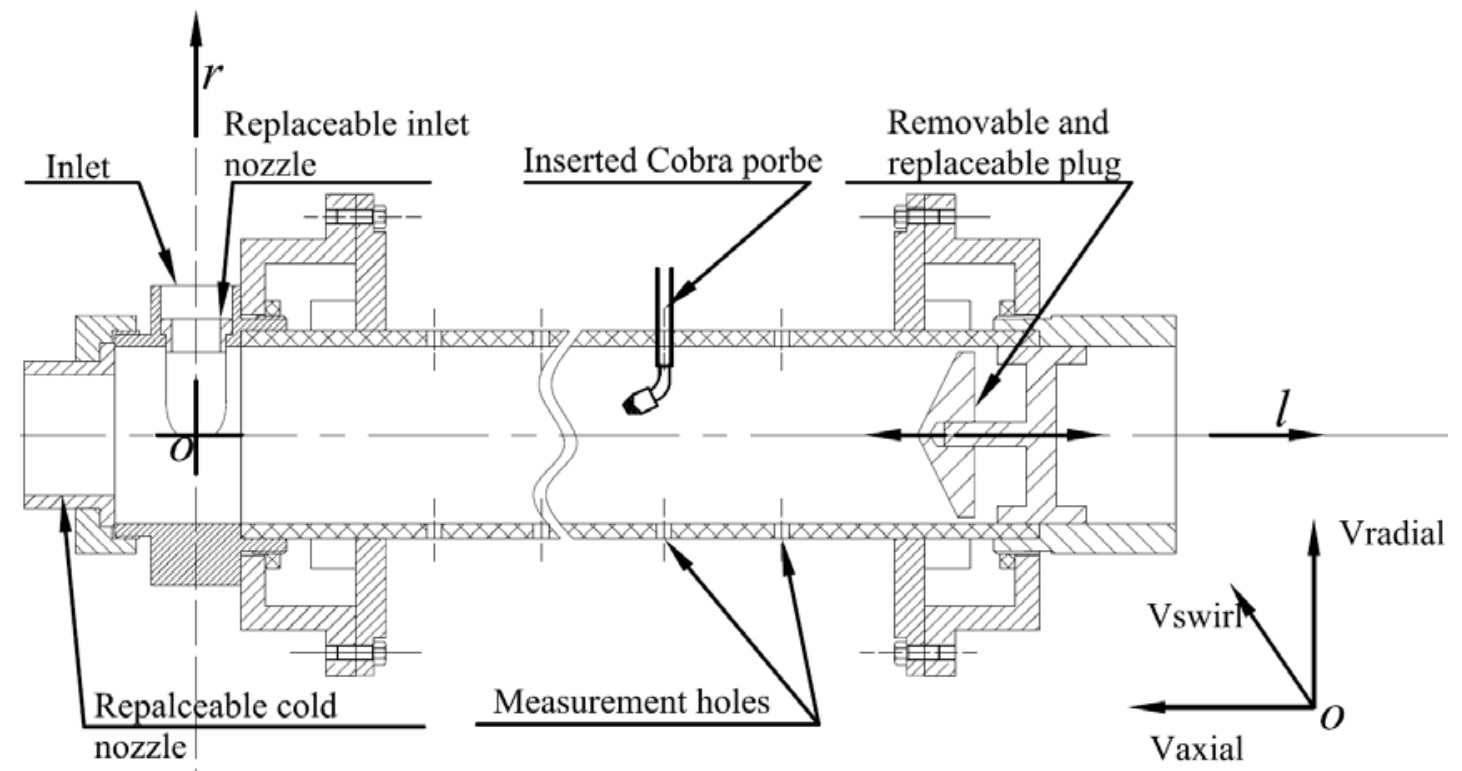

Figure 2.Experimental apparatus of measuring the flow properties in the large-scale vortex tube

Due to measurement range limitations, the cobra probe could only be used for the measurements of 3-D velocity when the flow velocity was between $2 \mathrm{~m} / \mathrm{s}$ and 50 $\mathrm{m} / \mathrm{s}$. For velocities higher than $50 \mathrm{~m} / \mathrm{s}$, the acceptance of data collected by the cobra probe was reduced to less than $80 \%$. Therefore, a Rotatable Pitot Tube (RPT) was employed for validation of the pressure and velocity measurements beyond the range 
measurable by the cobra probe. The structure and working principle of the rotatable Pitot tube are presented in Figure 3, which depicts a $1 \mathrm{~mm}$ tube with a $0.2 \mathrm{~mm}$ hole on one side and a pressure sensor connected at the other end. The RPT was rotating at a constant velocity during the measurement, and the measured surface pressure of the tube was recorded continuously. The direction and magnitude of the total velocity were calculated using the surface pressure distribution.

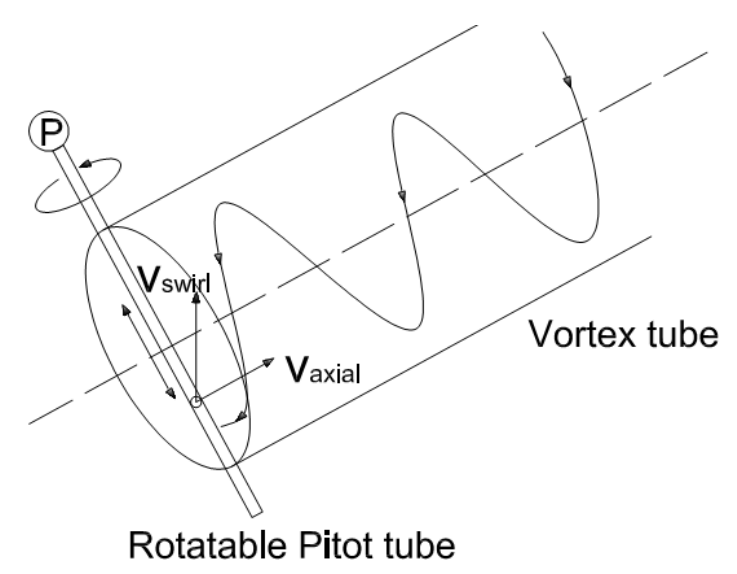

Figure 3. Structure and working principle of the rotatable Pitot tube used in the measurements of pressure and velocity in the vortex tube

The total temperature distribution along the vortex tube was measured using a Ttype thermocouple inserted into the tube through the available holes. Due to the tube dimensions and construction of the vortex tube, the temperature difference in this experiment was not as significant as it is in a commercial vortex tube. Considering this relatively small temperature change and the low Mach number of the flow in the tube, a recovery factor of 1 was assumed for the static temperature calculation. .

In the following sections, the hypothesis proposed in this research will be discussed along with similar concepts reported previously. Also, the flow properties obtained through measurements and calculations, including total and static pressures, total and static temperatures, 3-D velocity distributions and the local density, will be used to calculate the exergy density of the tube.

\section{Proposed hypothesis}


According to $[1,2,5]$, the hypothesis for temperature separation in a vortex tube is as follows: the vortical flow generated by the tangential injection moves to the hot end and the peripheral part escapes from the hot exit at a temperature higher than the inlet temperature. As reported in $[2,5]$, the irrotational vortex near the hot end indicates an increase in the centrifugal force on the central swirling flow, which leads to an outwards movement of the central fluid towards the tube wall. Hence, on its way moving to the cold end, the central flow spreads outwards, mixes with the peripheral flow and then turns back to the hot end again. This specific flow structure was first observed in [2] and named the multi-circulation. Because of the strong swirling flow in the rear part of the tube, a sub-structure could be found within the primary multi-circulation, which is presented by the smaller circles in the hot region shown in Figure 4. The temperature of the peripheral flow rises mainly due to the partial stagnation and mixing induced by the flow structure of the multi-circulation. The concept of the multi-circulation has also been proposed by other researchers. As demonstrated in Figure 5, the outwards movement of the central flow leads to its mixture with the peripheral flow and formation of the multi-circulation region [5].

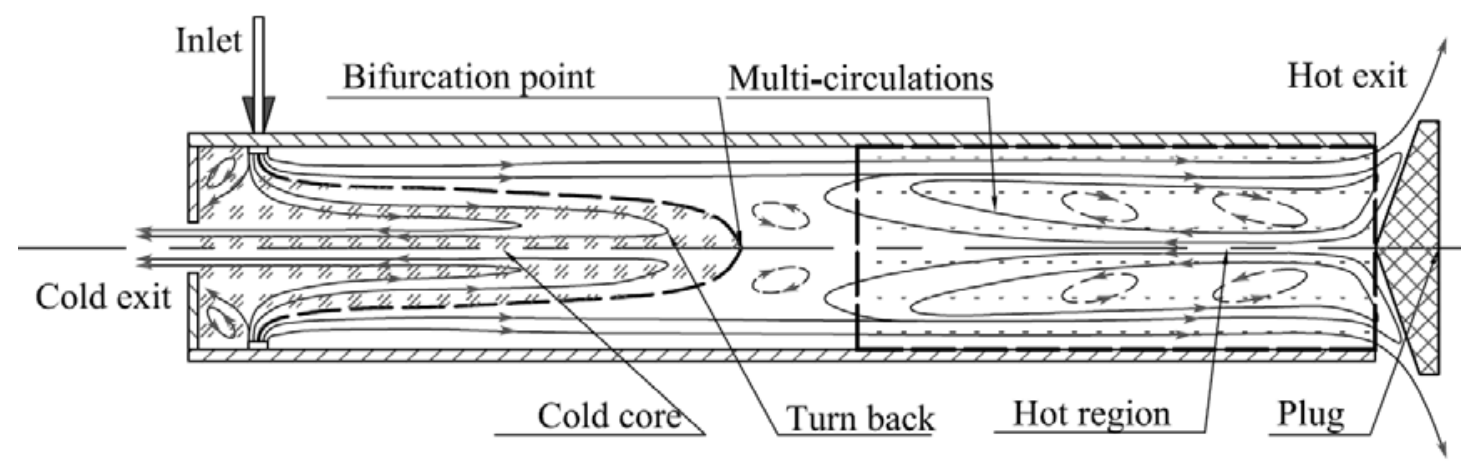

Figure 4. Flow structure inside a counter-flow vortex tube 


\section{NOTE:}

This figure/table/image has been removed to comply with copyright regulations. It is included in the print copy of the thesis held by the University of Adelaide Library.

Figure 5. Hypothesized flow behaviour in a vortex tube according to Crocker et al. [6]

The inner part of the flow moves towards the hot end and turns back in the front part of the tube. The flow in this region expands due to the lower pressure in the central part of the tube and then it escapes from the cold nozzle at a temperature lower than the supplied air. A similar description of the flow pattern in the front part of the tube was also reported by other researchers. M ore specifically, it was stated that part of the swirling flow turns back before reaching the hot end and leaves from the cold nozzle [7-12]. Figure 6 presents a proposed flow pattern in a vortex tube [9], in which the turning back of the peripheral flow in the front part of the tube is shown. Some of the inner flow might mix with the multi-circulation to form small vorticities that separate the cold flow from the hot flow. These small vorticities are also shown in Figure 4 and are located between the cold core and the hot region. As stated above, the lowest temperature is found at the centre of the tube near the injection point, and after mixing with other parts of the cold flow, the temperature of the exhausted stream from the cold nozzle rises by a small amount. The increased temperature of the exhausted gas from the minimum temperature in the central region of a vortex tube was found in [13], and the minimum temperature at $(r, I)=(0,0)$ has also been reported in both experimental and numerical investigations [13-17]. 


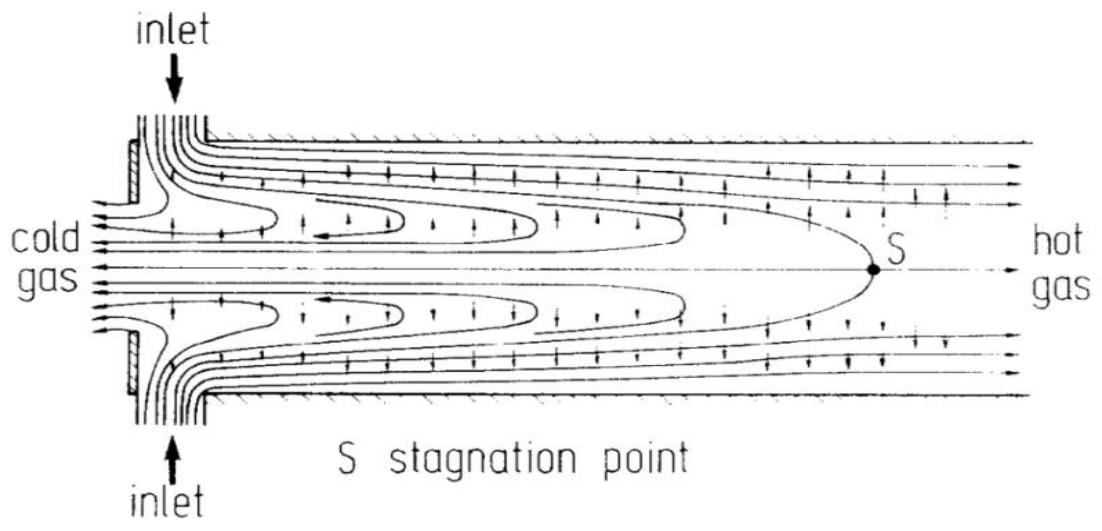

Figure 6. Proposed flow pattern in a counter-flow vortex tube [9]

\section{Exergy analysis inside the vortex tube}

In order to verify the above hypothesis, an energy analysis inside the vortex tube has been carried out. Through clarification of the energy transfer between different flow layers, a reasonable explanation can be revealed. If there is only a small amount of energy transferred between layers, the temperature separation in a vortex tube can be considered to be a result of a change in a certain variable such as pressure or velocity. Then the temperature drop of the cold air is the result of a sudden expansion near the entrance (or lower pressure region in the central part of the tube), and the temperature rise of the hot air is the result of the mixture and partial stagnation of the multi-circulation near the hot exit. Furthermore, if the energy transfer between different flow layers is significant, then the temperature separation in the tube might be caused by secondary circulation and angular momentum transportation by the internal friction between different layers of flow. The hypothesis offered in this research proposes that the hot and cold streams from the vortex tube are the result of a pressure drop, and that the mixture and partial stagnation are caused by the multicirculation. To validate the hypothesis, the exergy density of the internal flow is calculated.

Exergy within a control volume describes the maximum available energy and is calculated as follows:

$$
E x=\iiint\left(h+\frac{v^{2}}{2}+g z\right) \rho d V-\iiint(T s) \rho d V
$$


The exergy within a control volume consists of the total energy and entropy, i.e., the first and second components of the equation. The total energy within a control volume consists of three parts, i.e., enthalpy, kinetic energy, and potential energy. In fluid dynamics, the kinetic energy within a control volume consists two parts, as shown in the equation below:

$$
e_{k}=e_{k, \text { Mean }}+e_{k, \text { Turbulence }}
$$

Here, $e_{k, \text { Mean }}$ is the average kinetic energy within the flow and $e_{k, \text { Turbulence }}$ is the kinetic energy in the turbulence of the flow. The total kinetic energy within a control volume is calculated as follows:

$$
e_{k}=\frac{1}{2} \bar{v}^{2} d m+\frac{1}{2} v^{\prime 2} d m=\frac{1}{2} \bar{v}^{2} d m+\frac{3}{2}\left(\bar{v} I_{u v w}\right)^{2} d m
$$

Here, $d m$ is mass contained within the control volume, $\bar{v}$ is the time-averaged overall velocity, $v^{\prime}$ is time-varying velocity fluctuation, and $I_{u v w}$ is the overall turbulence intensity, respectively.

Gravitational potential energy of the mass within the control volume is given as:

$$
e_{g}=d m g z
$$

Here, $g$ is gravitational acceleration and $z$ is the net height.

The enthalpy within the control volume can be expressed as:

$$
h=d m C_{p}(\Delta T)
$$

Here, $C_{p}$ is the specific heat at constant pressure and $\Delta T$ is the temperature difference between a local substance and a reference condition. In the study of a rotating air flow, the influence of gravity is generally ignored due to its small magnitude. Hence in this paper, the total energy within the control volume can be given as:

$$
e_{t}=h+e_{k}
$$


In analysing compressible flow, the entropy within the control volume is very useful and is thus included in the exergy analysis. The change of entropy is given as:

$$
d S=\frac{\delta Q}{T}
$$

Here $\delta Q$ is the infinitesimal transfer of heat to the control volume, $T$ is the equilibrium temperature of the system, and $d S$ is the entropy change. Also, a change in the entropy of the system can be calculated from the following relationship:

$$
T d s=d h-\frac{d p}{\rho}
$$

Here, $d h$ is the change of enthalpy, $d p$ is the pressure change and $\rho$ is the density within the control volume. For a process within the control volume, the entropy change can be calculated as follows:

$$
S_{i}-S_{o}=C_{p} \ln \frac{T_{i}}{T_{o}}-\boldsymbol{R} \ln \frac{P_{i}}{P_{o}}
$$

Here, $R$ is the gas constant, $T$ and $P$ represent the temperature and pressure, the subscripts " $i$ " and " $o$ " represent the instantaneous and reference conditions of a process. Thus, the exergy within the control volume can be derived from the abovementioned equations and expressed as:

$$
E x=C_{p}\left(T_{i}-T_{o}\right) d m+\left(\frac{1}{2} \bar{v}^{2}+\frac{3}{2}\left(v^{\prime} I_{u v w}\right)^{2}\right) d m-T_{o}\left(C_{p} \ln \frac{T_{i}}{T_{o}}-R \ln \frac{P_{i}}{P_{o}}\right) d m
$$

In order to carry out a detailed analysis of the exergy distribution inside a vortex tube, the control volume in the vortex tube is defined to be infinitesimal, and hence the properties inside the control volume are constant. In this method, the density of the local fluid can be used to calculate the exergy distribution inside the vortex tube instead of the mass within the control volume, and the result of this calculation is defined as the exergy density distribution. Therefore, the equation is written in the following form:

$$
e x_{i}=\rho_{i} C_{p}\left(T_{i}-T_{o}\right)+\rho_{i}\left(\frac{1}{2} \bar{v}_{i}^{2}+\frac{3}{2}\left(v^{\prime} I_{u v w}\right)_{i}^{2}\right)-\rho_{i} T_{o}\left(C_{p} \ln \frac{T_{i}}{T_{o}}-R \ln \frac{P_{i}}{P_{o}}\right)
$$


Here, $\rho_{i}$ is the density of local fluid and can be calculated using the following equation:

$$
\rho_{i}=\frac{P_{i}}{R T_{i}}
$$

Here, $P_{i}$ and $T_{i}$ are the local static pressure and static temperature, respectively. In the calculation of the exergy density, $C_{p}$ is the specific heat at constant pressure and is $1006 \mathrm{~J} / \mathrm{kg} \cdot \mathrm{K}$ in this paper according to the flow conditions where $T_{o}$ is the reference ambient temperature, $\bar{v}$ is the time-averaged velocity, $R$ is $286.9 \mathrm{~J} / \mathrm{kg} \cdot \mathrm{K}$, $P_{o}$ is the reference ambient pressure. Values pertaining to the standard atmosphere were used as the reference condition, where $T_{o}=293.15 \mathrm{~K}$ and $P_{o}=101325 \mathrm{~Pa}$. With these parameters and the flow properties reported in [5], the exergy density gradient in the vortex tube was calculated.

Figure 7 presents the calculated exergy density inside the vortex tube at different locations. It is shown that the exergy density in the peripheral region decreases dramatically from the inlet, which is caused by the filling of the central part of the vortex tube by the peripheral flow. At $0.05 \mathrm{~L}$, the decrease of the exergy density in the radial direction also indicates the formation of the cold core as presented in Figure 4. Towards the hot end, the radial gradient of exergy density becomes constant. Heat transfer from the wall of the tube to the ambient air causes a gradual decrease in exergy density from approximately $0.5 \mathrm{~L}$ to the hot end. As discussed in the proposed hypothesis, the outer layer of the peripheral flow at the hot end is exhausted from the hot exit and the inner part of the peripheral flow is turned back by the plug towards the cold end through the central region of the tube. Therefore, the lower exergy density of the central flow at the hot end $(0.95 \mathrm{~L})$ is caused by the turned-back central flow from the peripheral part. As the central flow moves to the cold end, the exergy density decreases and reaches the minimum value at the cold end. At the peripheral region of the flow, the slightly reduced exergy density near the hot end indicates that there is no energy transferred outwards and that the temperature rise near the hot end is mainly caused by the mixture and partial stagnation of the axial flow via the structure of multi-circulation. 


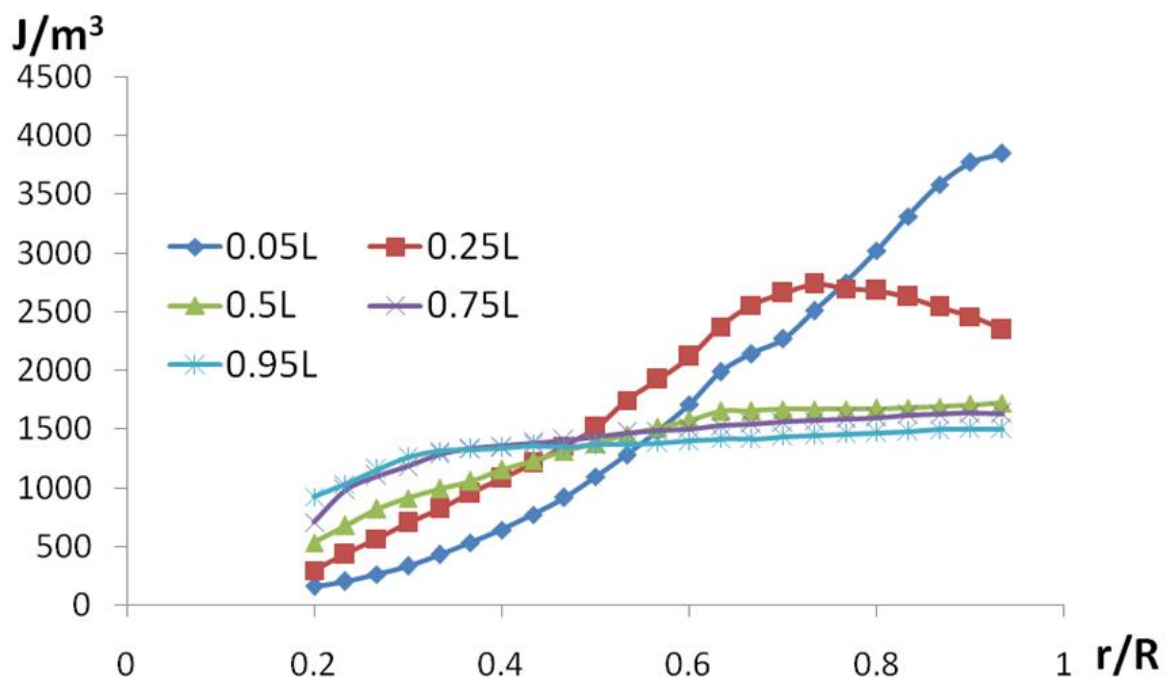

Figure 7. Exergy density at different positions of the vortex tube (current research)

To enable comparison between the tube performance in this research and in other studies, the exergy analysis was carried out using the flow properties reported in other experimental investigations. The experimental parameters used in this research are summarised below in Table 1. Note the large dimension of the investigated vortex tube, chosen to facilitate accurate flow and temperature measurement, and the variable input pressures that lead to a variable temperature difference. These variable parameters ensure the reliability of the exergy analysis.

Table 1. Parameters in several experimental investigations

Note: Gao used rectangular inlet nozzles (width $\mathrm{x}$ length $=1 \times 14 \mathrm{~mm}$ )

\begin{tabular}{ccccccc}
\hline & $\mathrm{D}$ & $\mathrm{L}$ & $\mathrm{D}_{\text {in }}$ & $\mathrm{L} / \mathrm{D}$ & $\mathrm{P}_{\text {in }}$ & $\Delta \mathrm{T}$ \\
\hline Gao (2005) & 40 & 2586 & $1 \times 14$ & 64.65 & 2.8 & 29 \\
Lay (1959) & 50.8 & 1143 & 12 & 22.5 & 2 & 35 \\
Lay (1959) & 50.8 & 1143 & 12 & 22.5 & 2.7 & 50 \\
Current & 60 & 1260 & 6 & 21 & 1.7 & 10 \\
\hline
\end{tabular}

The flow properties inside the vortex tube, including the total velocity, static temperature and static pressure, are obtained from [13], and are used to analyse the exergy gradients inside the tube (Table 2). 
Table 2. Flow properties collected from [13]

\section{NOTE:}

This figure/table/image has been removed to comply with copyright regulations.

It is included in the print copy of the thesis held by the University of Adelaide Library. 
It should be noted that the calculated exergy density distributions from the experimental studies shown above are not expected to match the data presented in this work due to the different parameters and reference conditions. However if the argument described above, based on the exergy density distribution is valid, then the trends of the exergy distribution in different experimental studies should be similar. For this purpose, a nondimensionalized value of exergy density is calculated using the relationship between the actual value of exergy density and the maximum value in the tube, which is defined as the exergy density ratio:

$$
\theta=\frac{e x_{i}}{e x_{\operatorname{Max}}}
$$

NOTE:

This figure/table/image has been removed to comply with copyright regulations. It is included in the print copy of the thesis held by the University of Adelaide Library.

Figure 8. Exergy density distribution along a vortex tube (after Gao, 2005)

Figure 8 presents the exergy density ratio $(\theta)$ along a vortex tube calculated as described above using experimental data from [13]. The kinetic energy of the turbulent component of the flow inside the vortex tube is ignored due to the minimal turbulence in the experiment and also because of its small magnitude in the calculation (generally less than 1\%). It is seen from Figure 8 that the exergy density in the peripheral region decreases from the cold end to the hot end and the exergy density in the central region decreases from the hot end to the cold end. The trend of the variation of the peripheral exergy density in the hot region of the tube agrees well with that found in the current research. The gradual decrease of the peripheral exergy density from $0.5 \mathrm{~L}$ indicates that there is little energy transferred outwards to the 
peripheral flow. Hence, the increase of the internal energy, which is indicated by the increase in temperature, is mainly caused by the energy transformation from the kinetic energy of the peripheral flow. This shows that the dominant reason for the temperature rise is the axial stagnation and mixture caused by the multi-circulation near the hot end. Also, in the cold part of the vortex tube, the dramatic decrease of the exergy density is caused by the filling of the central region of the tube by the peripheral fluid. It is reasonable to conclude that the temperature drop in a vortex tube is mainly due to the pressure drop caused by the sudden expansion of the inlet flow into the central region of the tube.

Figure 9 presents the calculated exergy density distribution based Lay's [18] experimental data. These distributions of the exergy density can be seen to be similar to those in Figure 8. A dramatic decrease of the peripheral exergy density in the cold region and uniform exergy density in the hot region can also be observed. The good agreement between the exergy density distributions in these experimental investigations provides direct evidence for the proposed hypothesis above.

\section{NOTE:}

This figure/table/image has been removed to comply with copyright regulations. It is included in the print copy of the thesis held by the University of Adelaide Library.

Figure 9. Exergy density distribution along a vortex tube (after Lay, 1959)

Moreover, the total exergy profile at the measurement location is calculated by integrating the exergy density as follows:

$$
E x_{t}=\int_{0}^{R} e x_{i} 2 \pi r d r
$$

To nondimensionalized this parameter, the following equation is used: 


$$
\theta_{t}=\frac{E x_{t, i}}{E x_{t, \max }}
$$

Here, $E x_{t}$ is the total exergy profile calculated by integrating the local exergy density from the centre to the periphery of the tube. $\theta_{t}$ is the ratio between the total exergy profile at the measurement location and the maximum exergy profile in the tube, which is near the point of injection. Figure 10 presents the calculated total exergy ratio based on data from the above-mentioned experimental investigations, from which similar trends of the exergy gradient are observed. It can be concluded from the figure that the dramatic decrease of the total exergy from the front part of the vortex tube agrees with the exergy analysis above. This highlights the increase of the entropy in the process of the expansion into the central region of the tube. The observation of uniform exergy profiles in the rear part of the tube supports the conclusion that little energy is transferred outwards to the peripheral flow. It is also evident that the temperature rise is mainly caused by the energy transformation from the kinetic energy of the peripheral flow. It is also noted that the total exergy profiles decrease at different rates and reach different steady levels near the hot end of the vortex tube. These differences in the exergy profiles are due to different experimental parameters.

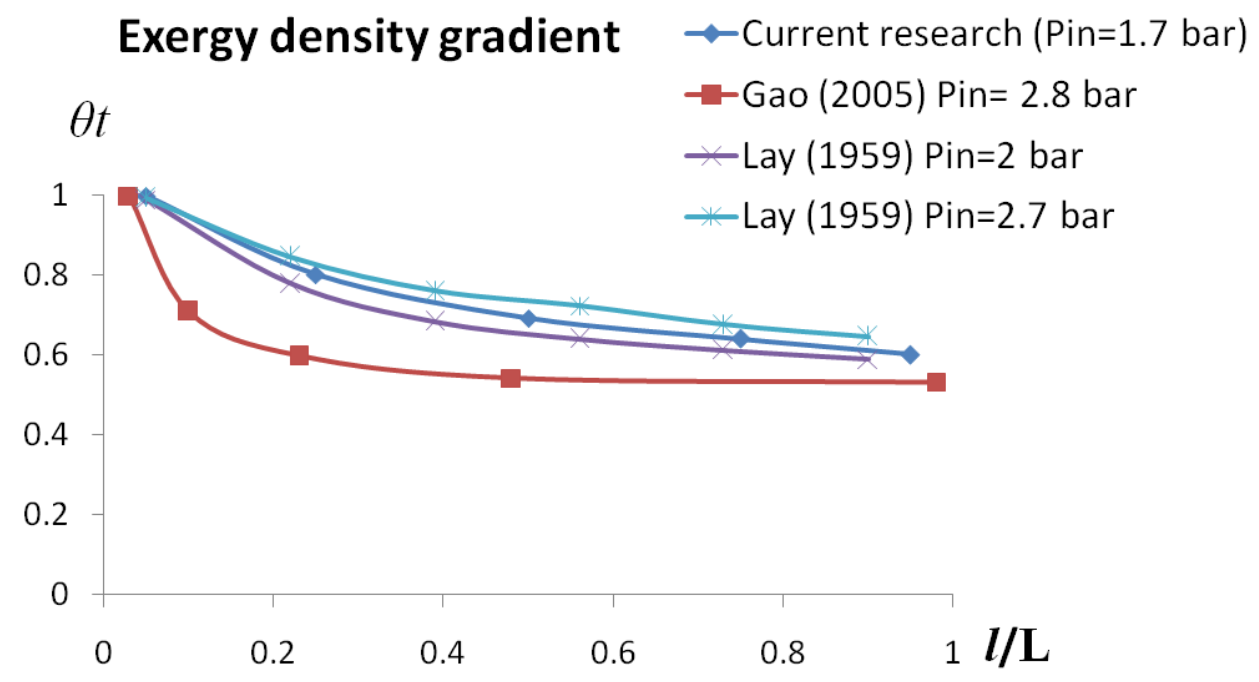

Figure 10. Total exergy density profiles along the vortex tube 


\section{Conclusion}

As part of the research on the vortex tube, this paper proposes a detailed hypothesis for the temperature separation based on previous studies [1, 2, 5]. Using detailed measurement results, an exergy analysis inside the tube is carried out, which provides positive evidence for the proposed hypothesis.

The temperature separation in a vortex tube is divided into two parts, i.e., temperature drop and temperature rise. The temperature drop at the cold end is caused by the sudden expansion in the front part of the vortex tube and the temperature rise at the hot end is the result of partial stagnation and mixture due to the multi-circulation flow structure in the rear part of the tube.

Using the experimental results from the current research and results from previous experimental investigations, an exergy analysis is carried out and used to support the proposed hypothesis from the point of view of energy transfer between different flow layers. The decrease of peripheral exergy density in the cold region is associated with the process of filling the central region of the tube, during which a sudden expansion occurs. Also, the uniform exergy profile in the rear part of the tube shows that there is no energy transferred outwards near the hot end and that the temperature rise is caused by the energy transformation from the kinetic energy of the peripheral flow. 


\section{Reference}

[1] Y. Xue, M. Arjomandi, R. Kelso, A critical review of temperature separation in a vortex tube, Experimental Thermal and Fluid Science, 34 (2010) 1367-1374.

[2] Y. Xue, M. Arjomandi, R. Kelso, Visualization of the flow structure in a vortex tube, Experimental Thermal and Fluid Science, 35 (2011) 1514-1521.

[3] B. Ahlborn, S. Groves, Secondary flow in a vortex tube, Fluid Dynamics Research, 21 (1997) 73-86.

[4] B.K. Ahlborn, J.M. Gordon, The vortex tube as a classic thermodynamic refrigeration cycle, Journal of Applied Physics, 88 (2000) 3645-3653.

[5] Y. Xue, M. Arjomandi, R. Kelso, Experimental study of the flow structure in a counter flow Ranque-Hilsch vortex tube, International Journal of Heat and Mass Transfer, 55 (2012) 5853-5860.

[6] A.M. Crocker, G.L. Sutphin, D.V. Cassisi, C. Knowlen, R.F. Weimer, Investigation of enhanced vortex tube air separators for advanced space transportation, in: 40th AIAA/ASME/SAE/ASEE Joint Propulsion Conference and Exhibit, Fort Lauderdale, FL, 2004.

[7] A.J. Reynolds, A note on vortex-tube flows, Journal of Fluid Mechanics, 14 (1962) 18-20.

[8] C.U. Linderstrøm-Lang, Gas separation in the Ranque-Hilsch vortex tube, International Journal of Heat and Mass Transfer, 7 (1964) 1195-1206.

[9] K. Stephan, S. Lin, M. Durst, F. Huang, D. Seher, An investigation of energy separation in a vortex tube, International Journal of Heat and Mass Transfer, 26 (1983) 341-348.

[10] N.F. Aljuwayhel, G.F. Nellis, S.A. Klein, Parametric and internal study of the vortex tube using a CFD model, International Journal of Refrigeration, 28 (2005) 442-450.

[11] U. Behera, P.J. Paul, S. Kasthurirengan, R. Karunanithi, S.N. Ram, K. Dinesh, S. Jacob, CFD analysis and experimental investigations towards optimizing the parameters of RanqueHilsch vortex tube, International Journal of Heat and Mass Transfer, 48 (2005) 1961-1973. 
[12] U. Behera, P.J. Paul, K. Dinesh, S. Jacob, Numerical investigations on flow behaviour and energy separation in Ranque-Hilsch vortex tube, International Journal of Heat and Mass Transfer, 51 (2008) 6077-6089.

[13] C. Gao, Experimental Study on the Ranque-Hilsch Vortex Tube, in, Technische Universiteit Eindhoven, 2005.

[14] O.V. Kazantseva, S.A. Piralishvili, A.A. Fuzeeva, Numerical simulation of swirling flows in vortex tubes, High Temperature, 43 (2005) 608-613.

[15] S. Eiamsa-ard, P. Promvonge, Numerical investigation of the thermal separation in a Ranque-Hilsch vortex tube, International Journal of Heat and Mass Transfer, 50 (2007) 821832.

[16] T. Farouk, B. Farouk, A. Gutsol, Simulation of gas species and temperature separation in the counter-flow Ranque-Hilsch vortex tube using the large eddy simulation technique, International Journal of Heat and Mass Transfer, 52 (2009) 3320-3333.

[17] A. Secchiaroli, R. Ricci, S. Montelpare, V. D'Alessandro, Numerical simulation of turbulent flow in a Ranque-Hilsch vortex tube, International Journal of Heat and Mass Transfer, 52 (2009) 5496-5511.

[18] J.E. Lay, An experimental and analytical study of vortex-flow temperature separation by superposition of spiral and axial flow, part I, Trans. ASME J. Heat Transfer, 81 (1959) 202212. 


\subsection{The working principle of a vortex tube}

International Journal of Refrigeration (Under review) 


\title{
Statement of authorship
}

\section{The working principle of a vortex tube}

\author{
Yunpeng Xue (candidate)
}

Performed experimental work, analysed data and wrote manuscript.

Signed:

Date

Dr. Maziar Arjomandi (Principal Supervisor)

Supervised the research and contributed in academic discussion and manuscript review

Signed: _ Date

Associate Professor Richard Kelso (Co-supervisor)

Supervised the research and contributed in academic discussion and manuscript review

Signed:

Date 


\title{
The working principle of a vortex tube
}

\author{
Yunpeng Xue, Maziar Arjomandi and Richard Kelso \\ School of Mechanical Engineering, the University of Adelaide \\ South Australia 5005, Australia
}

\begin{abstract}
The generation of cold and hot streams from a single injection in a vortex tube has been investigated by many researchers, aiming to define the primary reasons for the separation. This paper reports a detailed description of the flow behaviour inside a vortex tube and addresses the mechanism for the generation of cold and hot streams in a vortex tube, which is confirmed by different experimental methodologies, including visualization of the flow structure in a water-operated vortex tube, and measurement of velocity profiles in both water- and air-operated vortex tubes. Exergy analysis of the flow properties in an airoperated vortex tube indicates that there is no outward energy transfer in the hot region of the vortex tube. Furthermore, the governing factor for the temperature rise is attributed to the stagnation and mixture of the flow structure. Estimation of the theoretical temperature drop based on the pressure gradient of a forced vortex flow is addressed in this paper, and good agreement with the experimental results was observed. Based on the proposed mechanism, the predicted performance of the vortex tube with variable geometrical parameters, were congruent with the experimental results, underpinning the validity of the proposed mechanism.
\end{abstract}

Keywords: Ranque effect, exergy analysis, multi-circulation, thermal separation, vortex flow

\section{Introduction}

A vortex tube is a thermal device, which generates two streams at different temperature from a single injection. Injected into the vortex tube tangentially, the compressed gas is then divided into two parts and exhausted from the exits at temperatures lower and higher than the inlet gas, respectively. In this way, cold and hot streams are generated by only the vortex tube without any additional components. Figure 1 shows the structure of a counter-flow vortex tube as well as the proposed 
flow behaviour inside the tube. Importantly, as the vortex tube contains no other part inside the tube, the separation of two streams at different temperature by the vortex tube can only be attributed by the effects of fluid dynamics. In previous investigations, the vortex tube has been shown to facilitate in cooling, heating and mixture separation [1]. On comparison with other industry-based technologies, the significant advantages of the vortex tube, such as having no moving parts, being small, low in cost, maintenance free and having adjustable instant cold and hot streams, encourage the on-going investigations into the mechanism of this simple device, with the objective of improving the tube performance and identifying of the primary factors underlying its operation.

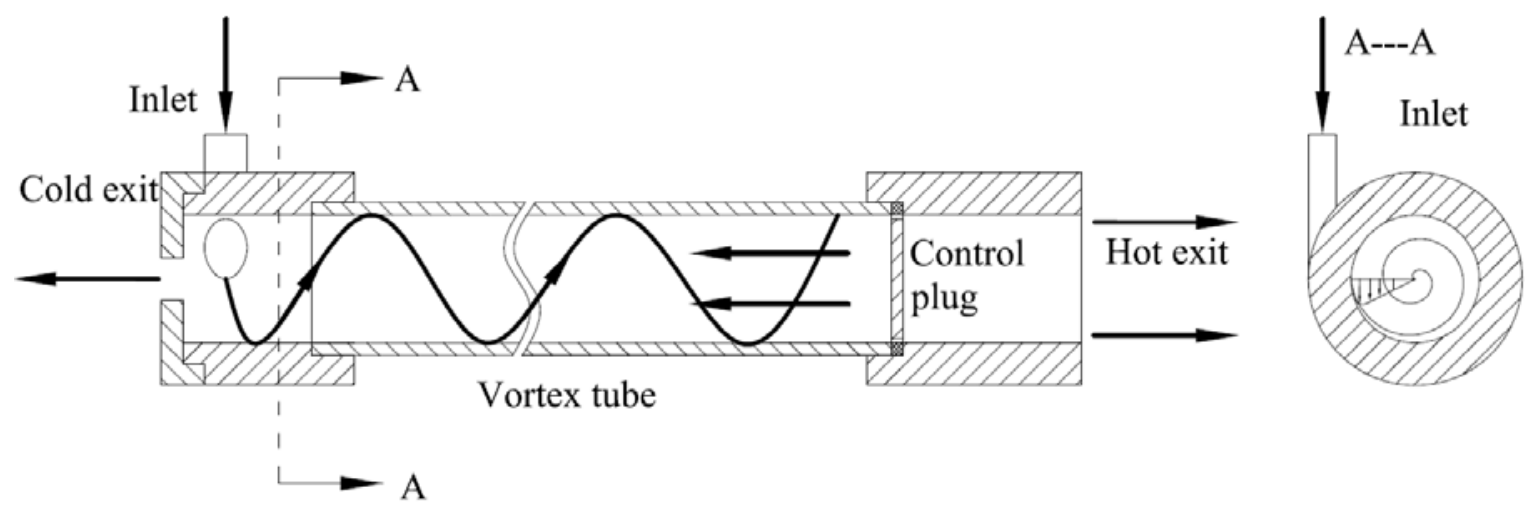

Figure 1. Flow structure in a counter-flow vortex tube

Several hypothesises have been proposed for the basic of the temperature separation, but a well-accepted explanation has not been forthcoming due to the complex flow mechanisms inside a vortex tube. In 2012, Liew et al. [2] reported that the adiabatic compression and expansion caused by the turbulent eddies, form the basic for the temperature separation, and provide a theoretical prediction of the temperatures based on the pressures at the exits. However, according to previously published work by Xue et al. [1], which summarised and analysed the available explanations, compression of the working fluid cannot be considered as the reason for the temperature rise, because the pressure inside a vortex tube is always lower than the inlet pressure. This is well supported by the measured pressure distribution along the vortex tube in both this [3] and other research work [4-6]. 
This paper reports a novel explanation for the generation of hot and cold streams in a vortex tube and is based on partial stagnation and mixture due to the nature of the multi-circulation occurring in the rear part of the tube, and the pressure gradient near the injection port. This proposed explanation is validated from a series investigations based on the analysis of flow behaviour and the properties inside the vortex tube $[1,3,7]$.

Visualizations of the flow structure, and measurements of the velocity components in both air- and water-operated vortex tubes, show that the proposed flow structure forces the flow to be divided into two streams at different temperatures. The validity of the proposed explanation is also supported by comparison of the estimated temperature drop and its correlation with experimental data in the cold part of the tube. Exergy analysis performed along the tube also shows that there is negligible energy transfer between different flow layers, which indicates the energy transformation of the peripheral flow is the primary factor in facilitating the temperature rise via the proposed flow structure. The working principle of the vortex tube is discussed in relation to the proposed mechanism, and is validated by the significant correlation existing between the theoretical value and experiment results, which are obtained in the course of this study.

\section{The cooling effect in a vortex tube}

The cooling effect of a vortex tube is identified in this research as the result of the sudden expansion of the working fluid near the injection port. When the fluid is injected into the vortex tube, the main part of the fluid rotates and moves along the periphery towards the hot end. Near the injection point, the inner part of the peripheral flow turns back and moves towards the cold exit. A cold core is formed near the injection due to the pressure gradient of the forced vortex, and the temperature drops due to the decreased pressure of the working fluid in this cold core. The flow behaviour in the cold part of a vortex tube can be seen in Figure 2, which shows the inwards turn back of the inner flow and the cold core. This explanation for the cooling effect is then validated in the following sections. 


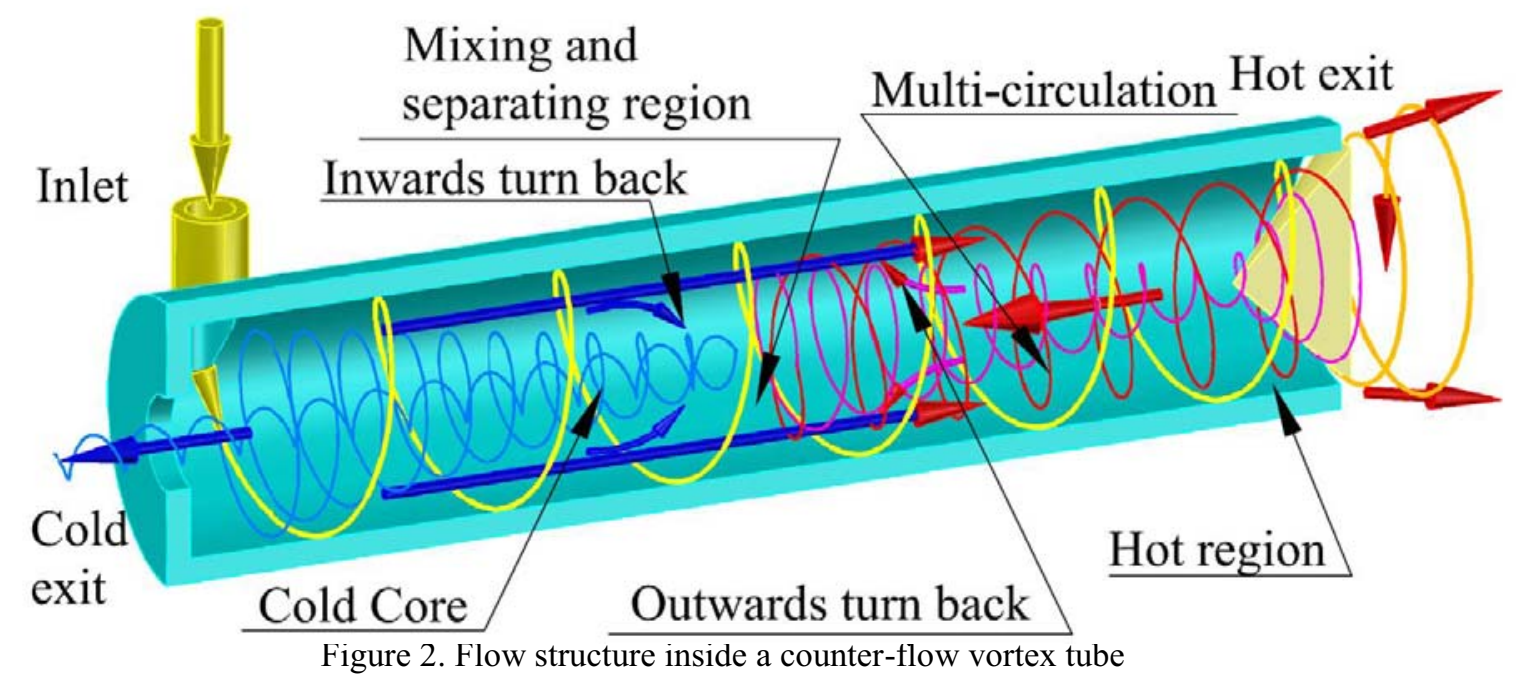

\subsection{Validation of the flow structure}

The explanation for the cooling effect is confirmed by the agreement between the hypothesised and experimentally observed flow structure. Visualization of the flow structure inside a water-operated vortex was conducted using different visualization methods [7]. The detailed description of the flow structure was based on the observed movements of the injected tracing particles in the clear tube [7]. It was found using tracing particles that the inner part of the injected fluid turned back, moved to the cold end, and exhausted from the cold exit.

The proposed flow structure inside a vortex tube is also validated using the measured velocity profiles in an air-operated vortex tube [3], which are presented in Figure 3 with the hypothesised flow structure. The negative radial velocity near the injection port (at $0.05 \mathrm{~L}$ ) shows that the peripheral flow moves to the centre of the tube. This inwards movement of the peripheral flow results in the sudden expansion near the cold nozzle, which leads to the formation of a cold core due to the pressure drop. The inwards, turn-back flow, in the cold core is the reason for the two maximum values of axial velocity at $0.25 \mathrm{~L}$, which are away from the centre of the tube. The flow structure in the front part of the tube is also supported by the axial velocity distributions at $0.05 \mathrm{~L}$ where most of the fluid near the injection port is moving to the cold exit. 

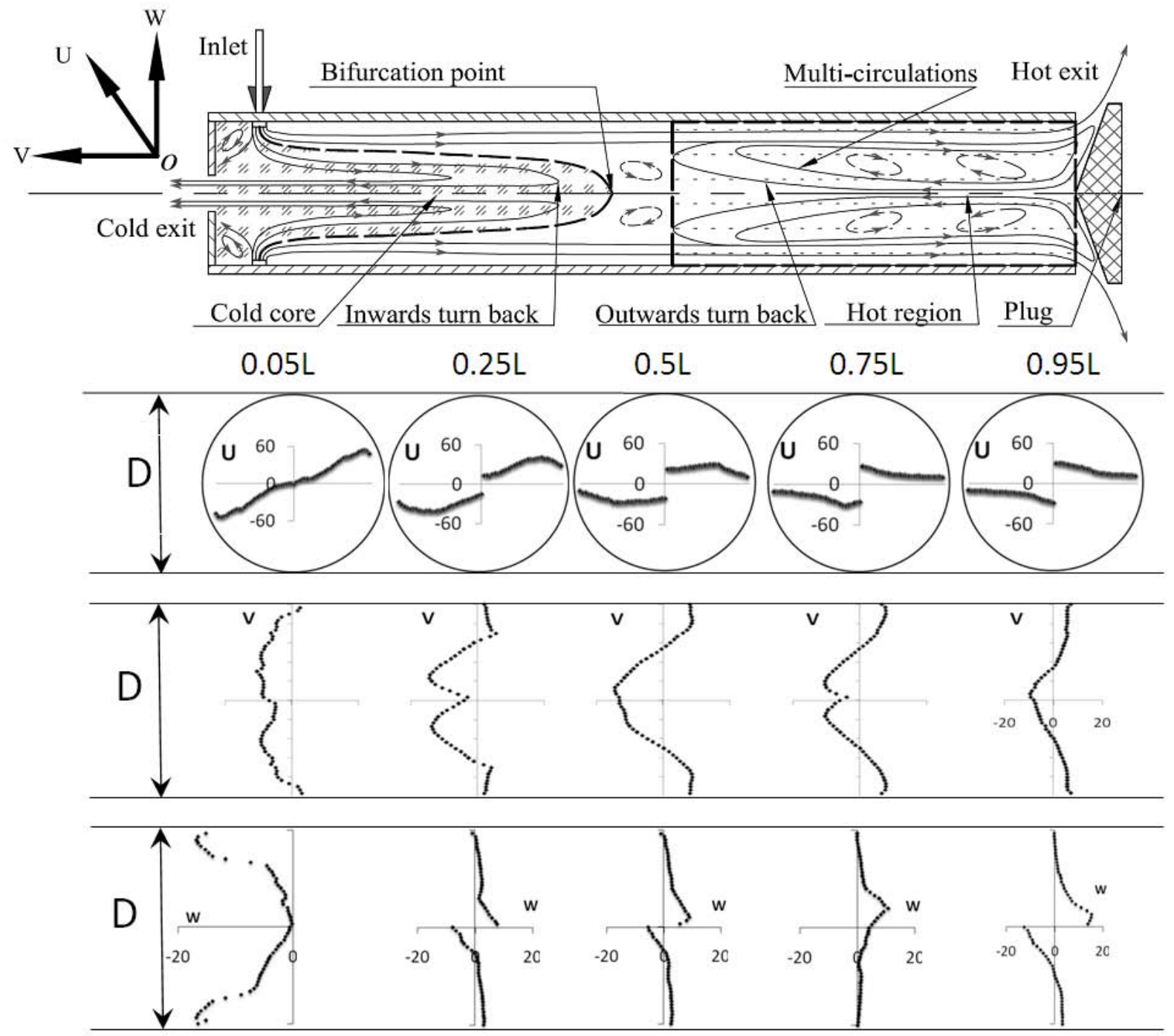

Figure 3. Hypothesized flow structure inside a vortex tube and velocity profiles at the corresponding position of the tube

(From top: ideal flow structure inside a vortex tube (3), swirl, axial and radial velocity profiles measured from $0.05 \mathrm{~L}$ near the injection port to $0.95 \mathrm{~L}$ near the hot exit, respectively.)

\subsection{Estimation of the temperature drop}

The temperature drop in a vortex tube is estimated based on the measured velocity profiles. The flow near the injection of a vortex tube performs as a forced vortex and the pressure distribution can be expressed as: 


$$
\frac{d p}{d r}=\rho \omega^{2}
$$

where, $\frac{\mathrm{dp}}{\mathrm{dr}}$ is the pressure gradient in the radial direction, $\rho$ is the local density of the flow, $\omega$ is the angular velocity, and $r$ is the radial location. Using the state equation:

$$
\rho=\frac{\mathrm{p}}{\mathrm{RT}}
$$

The pressure gradient is written as:

$$
\frac{R T}{p} \frac{d p}{d r}=\omega^{2} r
$$

For the adiabatic process, the relationship between the temperature and pressure of the control volume is:

$$
p^{1-\gamma} T^{\gamma}=C
$$

Substituting the relationship into the pressure gradient, it becomes:

$$
R C p^{-\frac{1}{\gamma}} d p=\omega^{2} r d r
$$

Integrate the equation and substitute peripheral properties, including density, static pressure and temperature at the inlet as $\rho_{i n}, P_{i n}$ and $T_{i n}$, respectively. The radius of the vortex tube and the heat capacity ratio of the fluid are $R_{t}$ and $\gamma$, respectively. The difference between the peripheral temperature and local temperature, which is also known as the temperature drop in a vortex tube, can be derived as:

$$
T_{i n}-T_{i}=\frac{\gamma-1}{2 R \gamma} \omega^{2}\left(R_{t}^{2}-r^{2}\right)
$$

As the geometrical parameters of the vortex tube vary, the flow at different temperatures in the vortex tube, mix and escape as the cold stream at a higher temperature than the minimum temperature calculated by equation 6 .

Recently, Polihronov and Straatman [8] performed a theoretical analysis of the angular propulsion effect in fluids and reported a temperature drop due to the 
adiabatic expansion in a vortex tube. A good agreement of the estimated temperature drops in their work and this research indicates the reliability of this estimation.

It is noted that the minimum temperature of a forced vortex is located at the centre. Hence, for a vortex tube, the maximum temperature drop occurs in the central part of the tube near the injection port. In order to confirm this estimation, experimental data from [4-6, 9] were compared with the calculated value. Due to the different experimental conditions, a nondimensionalized value of the temperature drop ratio $(\varepsilon)$ was calculated using the relationship between the actual temperature drop and the calculated maximum value using the above-mentioned method and is defined as:

$$
\varepsilon=\frac{\Delta T_{\text {actual }}}{\Delta T_{\text {estimated }}}
$$

The temperature drop ratio calculated from both this study and that of others' [46, 9] is shown in Figure 4. It is evident that the calculated maximum temperature drop is always greater than the experimental results. This can be explained by the reduction of the temperature drop due to the mixture of flow at different temperatures. The experimental results that are closer to the calculated value, indicates the better cooling performance of the vortex tube, since less mixing of the streams within the cold core leads to a lower temperature of the cold stream. Therefore, an estimation of the temperature drop based on the forced vortex assumption dictates the cooling capability of a vortex tube, and supports the notion that the pressure drop near the inlet is the main reason for temperature drop. 


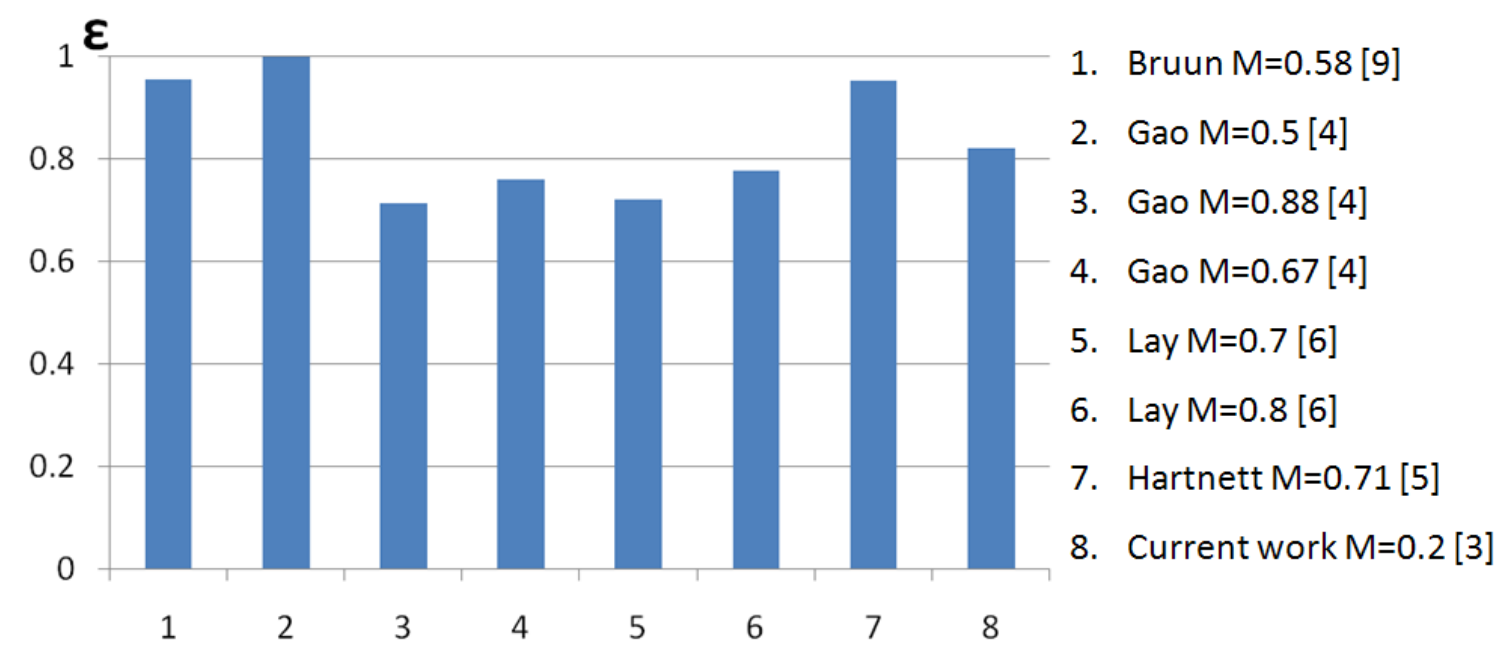

Figure 4. The temperature drop ratio from different experimental investigations

\section{The heating effect in a vortex tube}

The heating effect of a vortex tube could be induced by outwards energy transfer, including both thermal and kinetic energy, and partial stagnation of the swirling flow. As indicated in previous publication [1], the partial stagnation and mixture due to the flow structure located near the hot end, contributes significantly to the temperature rise in a vortex tube.

At the hot exit of a counter-flow vortex tube, the outer layer of the peripheral flow escapes from the small gap between the control plug and the tube as represented by the yellow helix in figure 2. The inner part of the flow is forced back through the central region of the tube (pink helix) by the plug. On its way towards the cold end, the swirling flow moves outwards to the periphery, mixes with the peripheral flow, and then returns back to the hot end (red helix). In this way the flow structure, termed multi-circulation, is formed and its partial stagnation and mixture is the primary factor for the temperature rise. The flow structure in the rear part of a vortex tube is also shown in Figure 2, as in the area of multi-circulation.

\subsection{Validation of the flow structure}


The actual flow behaviour near the hot end has been investigated in this study. Flow visualization near the hot end indicates that the outer layer of the peripheral flow continues its rotation and moves towards the hot exit [7], as shown in Figure 5. At the hot end, part of the outer layer flow is exhausted from the hot exit and the inner part of the flow is forced back by the control plug. The flow behaviour near the hot end of the tube was observed by tracing the movement of the injected particles within the forced-back flow. The position of a single particle in continuous frames from a video record is shown in Figure 6, in which the flow structure near the hot end was clearly appeared. It can be seen from the Figure that the focused particle moves upwards (towards to the cold end) with an increasing rotation radius and at certain position, the particle turns back to the hot exit again [7].

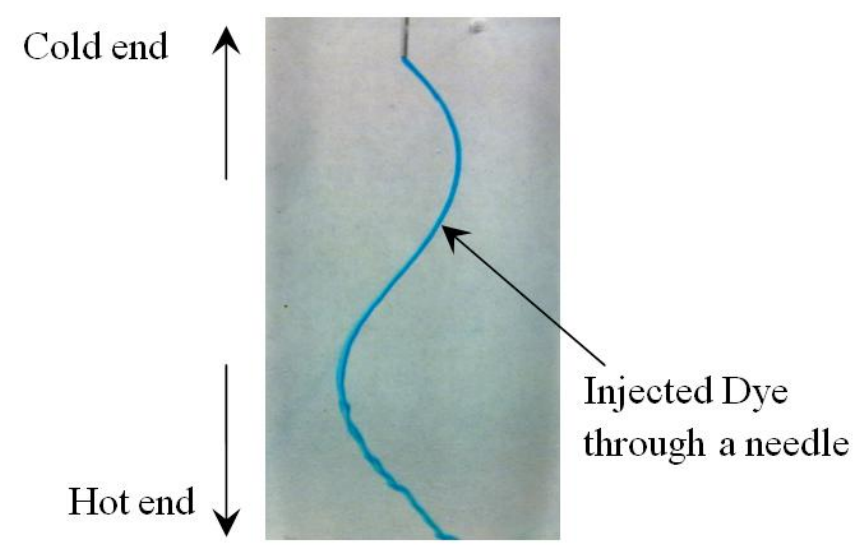

Figure 5. Helical movement of the flow inside the vortex tube

Thus, from the observations of each single particle, the flow behaviour near the hot exit can be described as: "Being forced back by the plug, the central rotating flow moves towards the cold end from the plug with an increasing rotating diameter. At a certain axial position of the tube, part of the central flow moves outwards in the radial direction, mixes with peripheral flow, and then turns back to the hot exit again. This flow structure, termed multi-circulation, supports the proposed explanation for the temperature rise, which are the partial stagnation and mixture induced by the multi-circulation. 


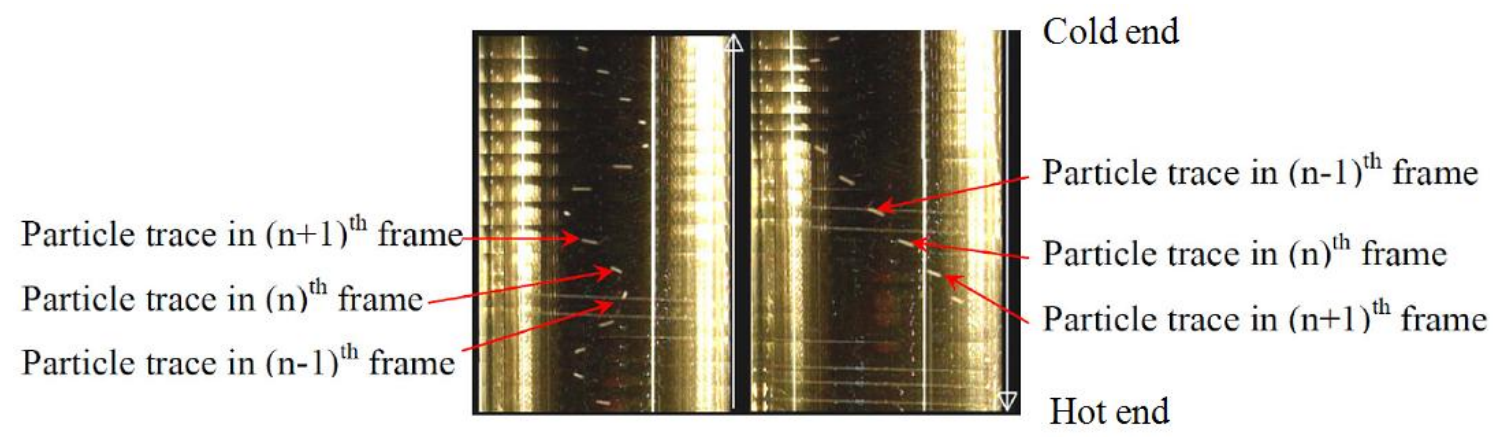

Figure 6. Position of the single particle in continuous frames

When the injecting needle was positioned at a proper location in the wateroperated vortex tube, the flow structure of multi-circulation was also visualized using dye injection. Figure 7 shows the recorded trajectory of the injected dye that moves towards the cold end, i.e., upwards of the figure, with an increasing rotating diameter. After a slight upwards movement, the injected dye then moves downwards to the hot end in the peripheral region. Therefore, this particular study shows the turn back of the central flow and indicates the formation of multi-circulation near the hot end of the tube.

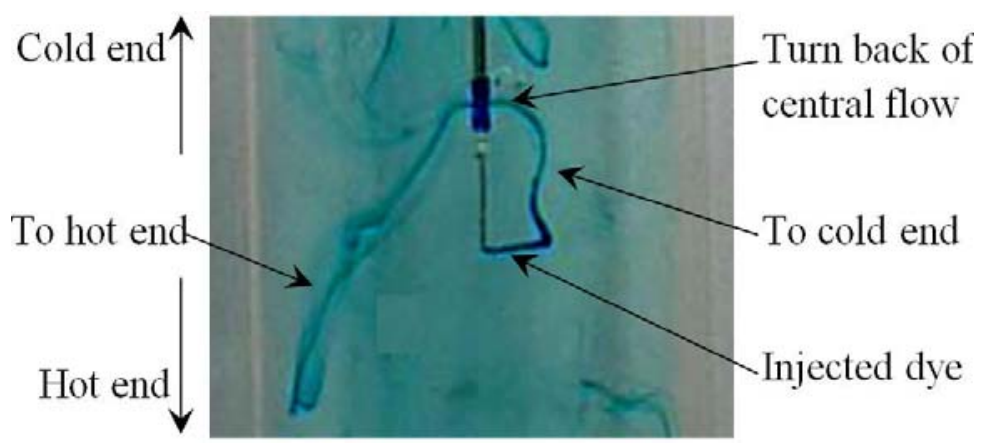

Figure 7. Visualization of the turn-back flow in the central region of the tube using dye

The flow structure of multi-circulation is also supported by the quantitive analysis of the velocity profiles presented in figure 3 . The maximum axial velocity at the hot end appears in the centre of the tube (at 0.95L) and shows the flow is forced back along the centre by the plug [3]. As the central flow moves to the cold end, the outwards radial flow induced by the increased centrifugal acceleration, causes the excursion of maximum axial velocity from the centre (at $0.75 \mathrm{~L}$ ). Corresponding to 
the same position, the formation of the multi-circulation is clearly apparent by the positive radial velocity, which indicates the outwards movement of the central fluid.

The swirl velocity distribution conducted from the flow visualization results [7] indicates an irrotational vortex appears near the hot end of the water-operated vortex tube. In an air-operated vortex tube [3], the swirl velocity distributions indicate a transformation from a forced vortex at the cold end to an irrotational vortex model at the hot end (Figure 3). The irrotational vortex flow near the hot end of a vortex tube, which leads to the outwards movement of the central flow and formation of the multi-circulation, would result in a greater centrifugal force in the central region. The structure of multi-circulation is also supported by the distribution of the volume flow rate along the water-operated vortex tube as reported in [7].

\subsection{Exergy analysis of the internal flow}

The core question in this investigation is the clarification of the energy transfer between different layers of flow [1]. If the energy transfer between layers is significant, kinetic energy transfer outwards due to the viscosity and turbulence of the flow should be considered as the reason for the separation. On the contrary, sudden expansion and partial stagnation are the primary factors in the temperature separation, in case of negligible energy transfer. Therefore, an exergy analysis, which defines the maximum available energy of the system, inside a vortex tube, was employed in order to validate the proposed explanation.

To conduct sensitivity analysis, an infinitesimal control volume is selected, which enables the calculation of exergy based on the result of measurements inside the vortex tube. In this case, the local density at the measurement position can be used in the exergy analysis instead of the mass of the control volume. Defined as exergy density, the calculated results indicate the exergy level of the flow inside the tube, from which energy transfer can be identified. The exergy density is calculated as:

$$
e x_{i}=\rho_{i} C_{p}\left(T_{i}-T_{o}\right)+\rho_{i}\left(\frac{1}{2} \bar{v}_{i}^{2}+\frac{3}{2}\left(v^{\prime} I_{u v w}\right)_{i}^{2}\right)-\rho_{i} T_{o}\left(C_{p} \ln \frac{T_{i}}{T_{o}}-R \ln \frac{P_{i}}{P_{o}}\right)
$$


Here, $\rho_{i}$ is the local density and calculated using the state equation, $C_{p}$ is the specific heat at constant pressure, $T$ and $P$ represent the static temperature and static pressure, respectively, the subscripts " $i$ " and " $O$ " represent, respectively, the instant and reference conditions of a process, $\bar{v}$ is the time-averaged total velocity, $v^{\prime}$ is the time-varying velocity fluctuating component, $I_{u v w}$ is the overall turbulence intensity, and $\mathrm{R}$ is the universal gas constant.

In order to draw a reliable conclusion form the exergy density analysis, flow properties inside the vortex tube were collected from this and other studies. It should be noted that the calculated exergy density distributions from these studies are different due to the different parameters and referencing conditions. Hence, a nondimensionalized value of exergy density calculated by the relationship between the actual value of exergy density and the exergy density at the inlet, is defined as:

$$
\theta=\frac{e x_{i}}{e x_{i n}}
$$

Note the exergy density at the injection inlet is also the maximum value of the exergy density of the flow in the vortex tube. The exergy density profiles along the vortex tube are presented in Figure 8, which are calculated using the experimental data from this study [3], as well as the data of Gao's [4] and Lay's data [6]. It can be seen from this figure that the exergy density of the peripheral flow decreases dramatically from the inlet, which is due to the filling of the inner part of the vortex tube from the peripheral flow. This distribution of exergy density indicates the existence and effect of the cold core in generating cold streams as discussed previously. In the peripheral region, the slightly decreased exergy density near the hot end indicates that there is little energy transferred outwards to the peripheral flow. The outwardly-directed energy transfer, including both thermal and kinetic energy components, cannot be treated as the primary reason for the temperature rise. Hence, partial stagnation and mixture of flow contributes greatly to the temperature rise via the structure of multi-circulation. 

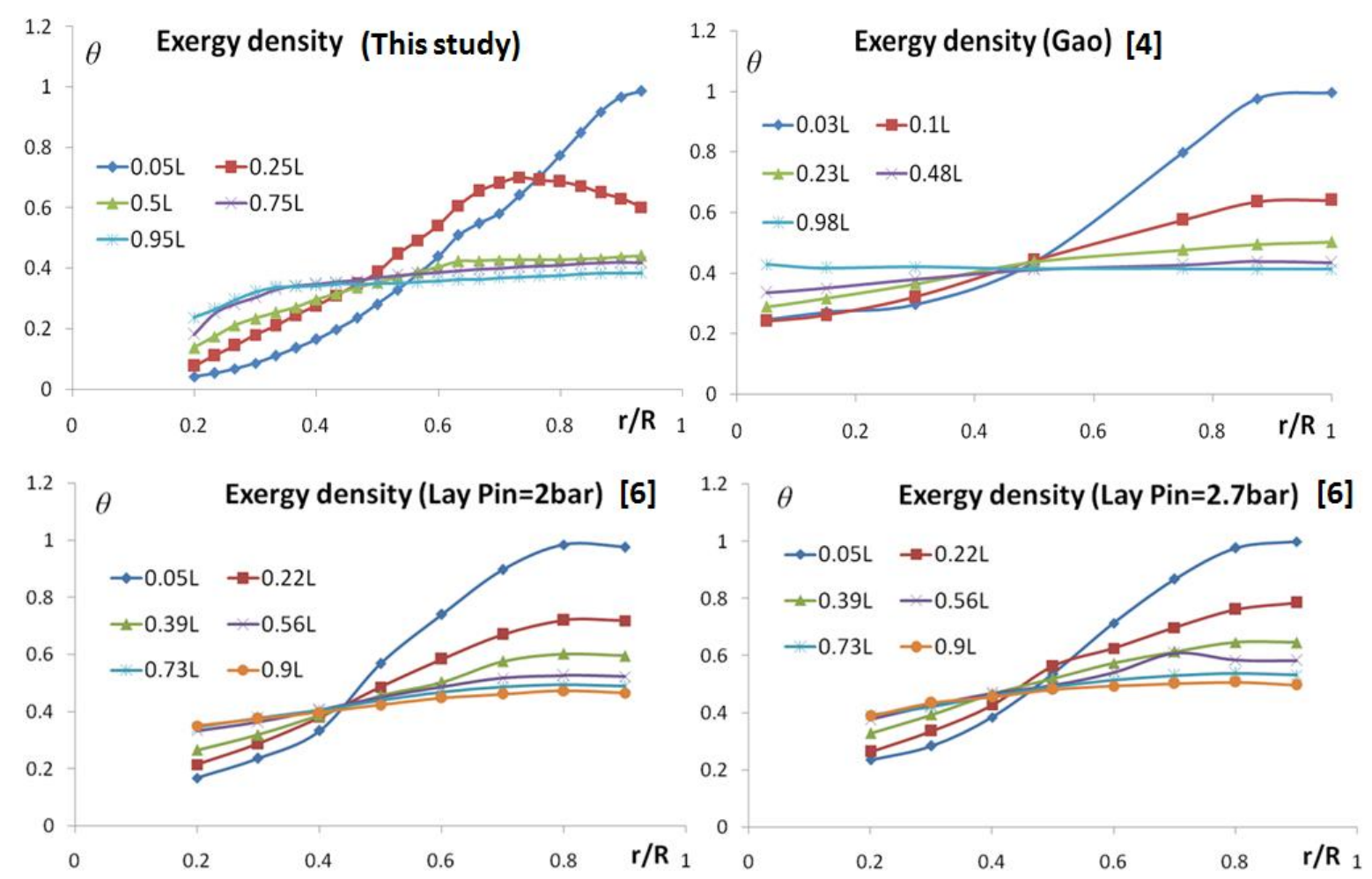

Figure 8. Exergy density at different positions of the vortex tube

\section{Working principle of a vortex tube}

Based on the discussions above, the flow behaviour inside a vortex tube is summarised in detail as shown in Figures 2 and 3. This basic on which the temperature separation in a vortex tube can be explained is as follows: When compressed air is injected into the tube from the tangential inlet, it forms a highly vortical flow and moves to the end of the tube. The inner part of the flow moves towards the hot end and turns back in front part of the tube. This part of the flow gets expanded due to the low pressure in the central part of the tube and escapes from the cold nozzle at a lower temperature than the injected air. Small amount of the inner flow mixes with the multi-circulation and forms small vorticities that separate the cold flow and multi-circulations. Due to the pressure gradient in a vortex tube, the lowest temperature will be found in the central part of the flow near the injection port. After mixing with other clod flow, which has been inwardly turned back, the minimum temperature of the exhausted stream from the cold nozzle will be found to be higher than the lowest temperature inside the tube. The cold and hot regions are 
also shown in the above figures, and between them is the mixing and separating region.

The peripheral part of the airflow escapes from the hot exit at a higher temperature than the inlet temperature and the inner part of the flow is forced back by the plug at the hot end. Due to the increase of the swirl velocity, the centrifugal force of the swirling flow increases and leads to the outwards flow of the central fluid. On its way to the cold end, the central flow moves outwards, mixes with the peripheral flow, and turns back to the hot end again. In this way, the central flow performs multiple circulations before being exhausted from the hot exit. Because of the strong swirling flow in the hot region of the tube, sub-cycles of the multicirculation might be found in the principal multi-circulation, which is represented by the dashed line in Figure 3. The temperature of the peripheral flow arises due to the partial stagnation and mixture induced by the multi-circulation. The maximum temperature should be found at the outwards turn back to the hot end of the central flow, i.e., about one third of the overall tube length distant from the hot end as discussed in [1]. The maximum temperature along the wall was also reported to be some distance from the hot exit $[10,11]$.

Based on this proposed flow behaviour, the working principle of a vortex tube at variable cold mass flow ratio, which is generally controlled by adjusting the hot end plug, is discussed below. To perform an accurate analysis of the working process inside a vortex tube, the flow pattern is divided into several regions (presented in Figure 9), which are the vorticities in the corner of the tube (1), the cold core (2), the peripheral flow (3), the mixing and separating region (4), and the hot region or multicirculation region (5).

The vorticities (1) in the corner of the tube are induced by the injected fluid and may be noticed as the secondary circulation when the cold exit is small [12]. When the cold exit of the vortex tube is larger than a critical value (i.e. $d_{c} / d_{t}=0.58$, here $d_{c}$ is the diameter of cold exit and $d_{t}$ is the diameter of the vortex tube [13]), all the flow moving to the cold end will be exhausted from that exit and there will be no flow being forced back to form the vorticities in the corner. 
The cold core region (2) indicated by the blue helix in figure 2, locates near the injection point, and it is the region where expansion occurs and the temperature drops due to the pressure gradient of the forced vortex. The minimum temperature inside a vortex tube is always found in the central part of the cold core opposite the injection port. The size of the cold core varies in the vortex tube with different experimental parameters.

The peripheral flow (3) represented by the yellow helix in figure 2, is that flow which moves in the peripheral layer and escapes from the hot exit. It mixes with the multi-circulations and leaves the tube at a higher temperature due to energy transformation from the kinetic energy of the peripheral flow, as discussed in the previous exergy analysis.

The mixing and separating region (4) may be found between the inwards turn back flow in the cold core, and the outwards turn back flow of the multi-circulation in a vortex tube. This region ensures the best performance of an ideal vortex tube by preventing the mixture of cold and hot flow regions. In a vortex tube, which is not designed properly, the mixing and separating region of the respective flow are not well delineated, and hence, this leads to a reduction in the separating performance.

The multi-circulation (5) locates near the hot end and causes the temperature rise of the working fluid in the process of stagnation and mixture with the peripheral flow. It is also indicated by the red and pink helix in figure 2, which show flow movement to the hot end and cold end, respectively. For a vortex tube with small hot exit, the central part of the multi-circulation region may move towards the cold end through the mixing region (4) and mix with the cold flow in the cold core region (2). Hence, the temperature drop of the cold flow is reduced by this mixing. 


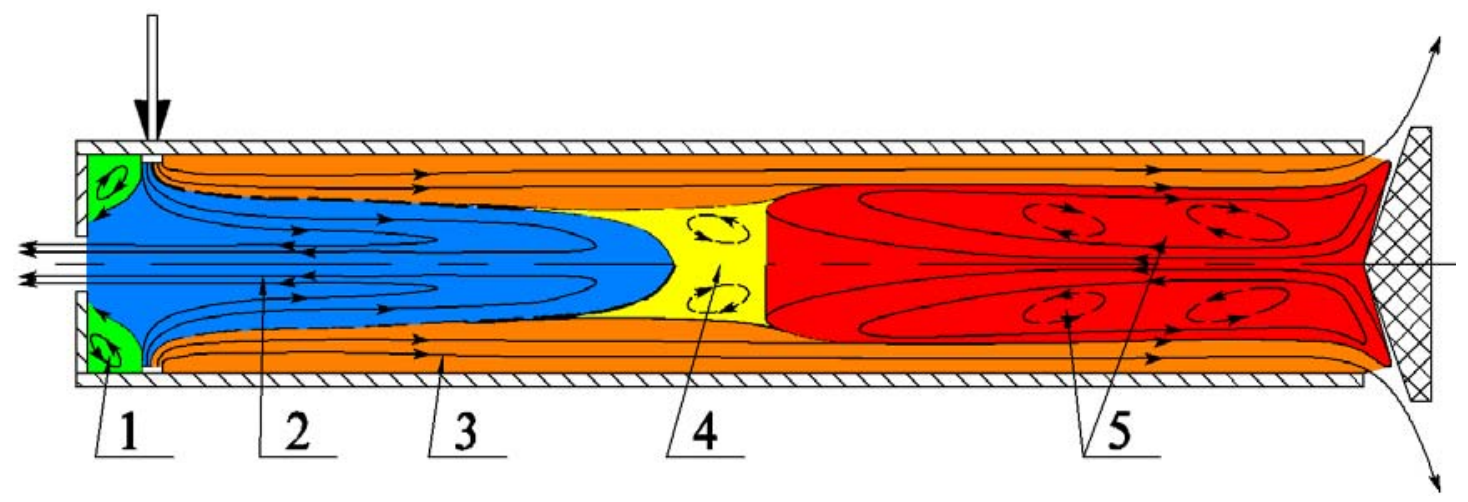

Figure 9. Divisional flow pattern inside a counter-flow vortex tube

When the hot exit of a vortex tube is relatively large, there will not be any flow forced back by the plug. Instead, all of the injected flow will escape from the hot exit. Due to the strong swirling flow, a low pressure region in the central part of the tube near the cold exit will be formed, reducing the extent of the temperature drop. Hence, entry of ambient air into the vortex tube through the cold exit by suction, can occur and may lead to more fluid from the hot end being exhausted than the injection. The inward suction of the ambient air has previously been reported by the authors [3] due to this low pressure region near the cold exit. The temperature of the exhausted gas will show a small increase due to the mixture and friction effect, which is weakened both by the temperature drop near the injection point and entry of ambient air by suction. The flow inside the tube will perform as a forced vortex and decay towards to the hot end. As shown in Figure 10, the flow behaviour in a vortex tube with a large hot exit, shows that only the peripheral flow (3) in the vortex tube, as well as the sucked ambient air moving along the centre exist under this condition. 


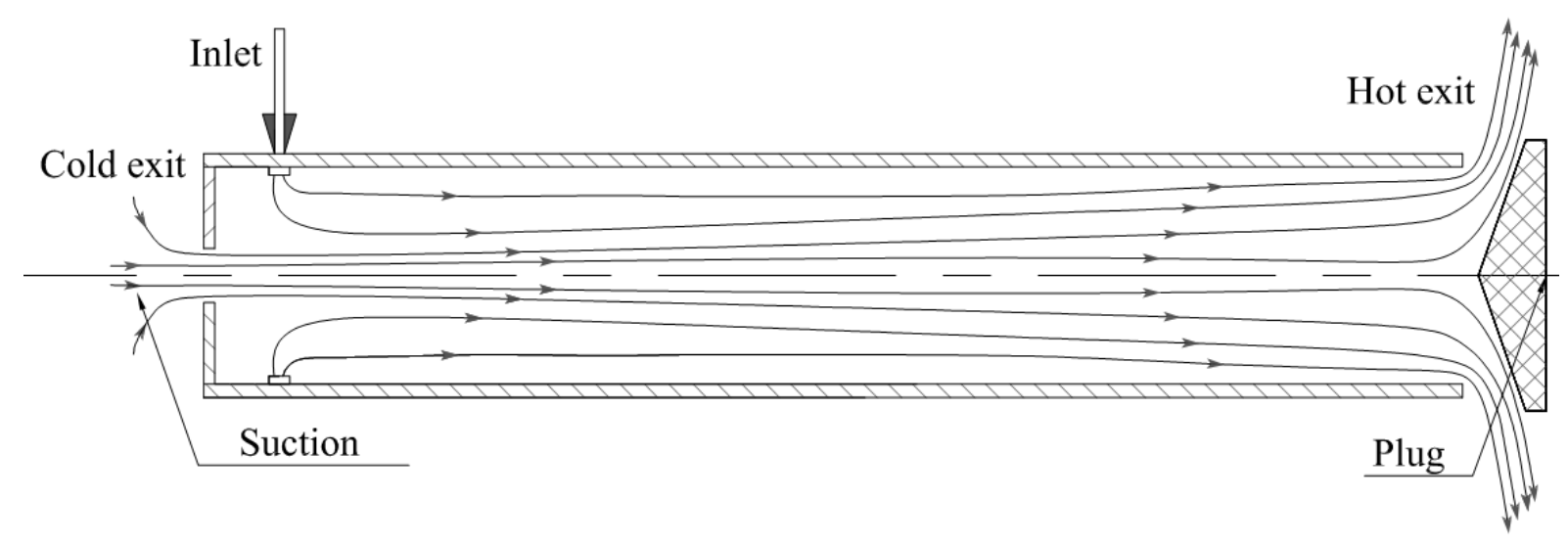

Figure 10. Flow structure in a vortex tube (1)

When the area of the hot exit is reduced, there will be some gas escaping from the cold exit and as a result, less gas leaves from the hot end. Part of the peripheral flow will be forced back by the plug and multi-circulation (5) with small scale will be formed. A transformation from forced vortex at the cold end to the irrotational vortex at the hot end will be observed. Partial stagnation and mixture due to this small scale multi-circulation causes a rise in the temperature of this flow, although the temperature in this region is lower than the maximum temperature generated by the vortex tube. The inner flow starts turning back because of the blockage by the multi-circulation. Temperature drops in the cold core (2) is caused by the pressure gradient in the tube, although there may be some suction of ambient air, which reduces the temperature drop at the cold exit, depending on the dimensions of the cold and hot exits. The vortex tube under this condition could generate cold and hot streams, and its likely flow structure inside the tube is shown in Figure 11.

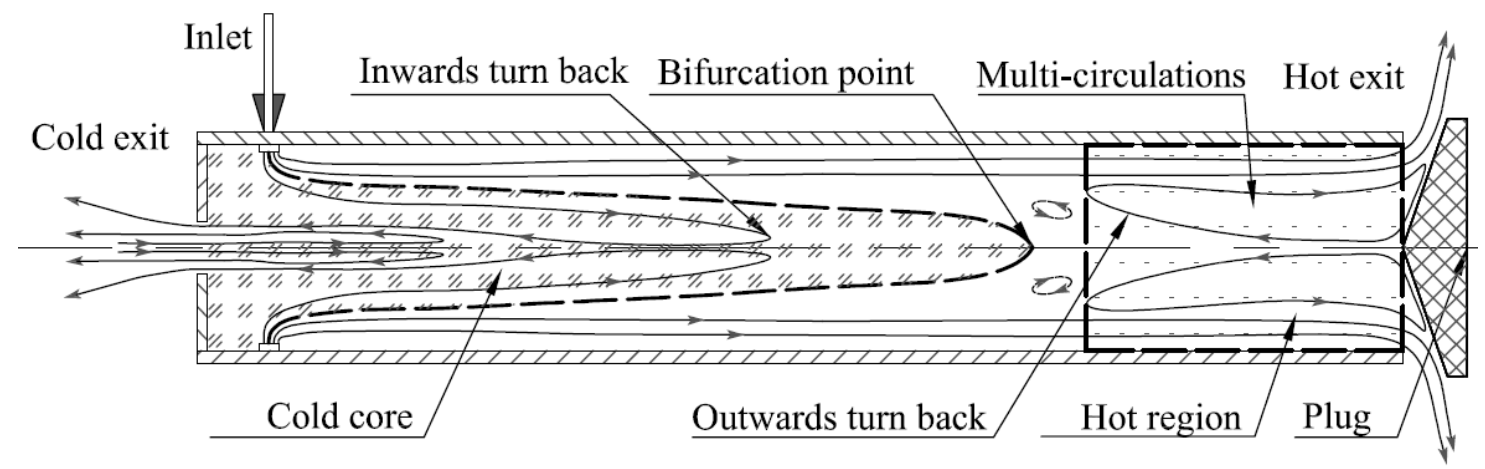

Figure 11. Flow structure in a vortex tube (2) 
Once the hot exit is further decreased, more cold stream and less hot stream will be exhausted from the vortex tube. A t certain cold mass flow ratio, the pressure at the cold exit becomes greater than the ambient pressure; hence suction at the cold exit will no longer be observed (shown in Figure 3). The temperature of the inner flow decreases due to the pressure gradient, and represents the minimum temperature within the vortex tube, and is the result of both the maximum volume of the cold core, and the lack of mixing the ambient air or the hot stream. Concomitantly, the temperature of the hot steam increases due to the increased scale of the multicirculation, which strengthen the effect of partial stagnation and mixture.

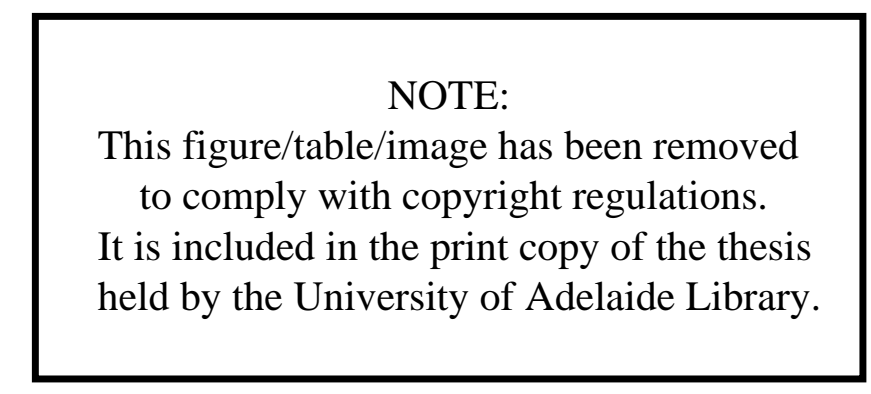

Figure 12. Flow structure in a vortex tube (4)

If the cold mass flow ratio keeps increasing, a concomitant increase of the multicirculation (5) and a decrease of the cold core region (2) would be expected as shown in Figure 12. Therefore, due to the smaller region for expansion and more gas in the cold region, the temperature drop of the cold stream would be reduced. As a result, the effect of partial stagnation and mixture is therefore strengthened by the increased scale of the multi-circulation, which leads to the increase of the hot stream temperature. Moreover, due to the decreased hot exit area, more gas will be forced back by the plug and subsequently, move to the cold end along the central part of the tube. Thus, part of the central flow may mix with the cold flow and escape from the cold exit. This mixture of two flows at different temperature causes the rise of cold temperature at the cold exit and weakens the cooling effect of the vortex tube.

In condition when the hot exit of a vortex tube is blocked, all the injected gas leaves from the cold nozzle, i.e. the cold mass flow ratio equals 1 , and the flow 
structure in the vortex tube is represented in Figure 13. It is seen from the Figure that the main part of the injected gas gets expanded in the cold core and is exhausted from the cold exit. In such a condition, part of the peripheral flow then moves to the hot end and forms the structure of multi-circulation in the rear part of the tube. Hence, the temperature of flow in the rear part of the tube still increases due to the partial stagnation and mixture in the multi-circulation region, which is indicated by the measured temperature of the tube wall at the hot end. It was reported in [14], that the temperature of the tube wall at the hot end had a 14 centigrade degree rise when the hot exit was blocked. As the cold core region decreased and the amount of the expanding stream increased, the temperature drop near the injection was not obvious as that in the vortex tube with other settings. The mixture of the high temperature stream coming from the multi-circulation region further reduced the temperature drop, which was reported as a 3.2 centigrade degree drop at the cold exit as reported in [14]. The tube performance when the hot end is blocked has also been reported in others' studies $[4,10,11,15]$.

\section{NOTE:}

This figure/table/image has been removed to comply with copyright regulations. It is included in the print copy of the thesis held by the University of Adelaide Library.

Figure 13. Flow structure in a vortex tube (5)

According to the proposed explanation, the parameters influencing the temperature change as a function of the variable cold mass ratios discussed above, is in agreement with the experimental results reported in [15]. With regard to the temperature drop of the cold stream at a cold mass ratio from 0 to 0.3 , the improving performance of the cooling effect may be caused by the decrease with the extent of ambient air suction, until such time the tube reaches its best cooling performance at a certain cold mass ratio. When the cold mass ratio is greater 0.3 , the temperature of the cold flow increases with the increasing cold mass ratio. This is mainly due to the 
reduction of the cold core region and the increase of the hot stream emanated from the multi-circulation region. The temperature of the hot stream increases with the increase of clod flow ratio or decrease of the hot flow ratio. Furthermore, the increasing temperature is caused by stronger stagnation and mixture due to the increasing region of multi-circulation as discussed above.

It should be noted that for a vortex tube having a cold mass ration greater than 0.8, i.e. the settings between Figures 12 and 13, the decreased extent of the peripheral flow moving to the hot end, leads to the formation of a weaker region of multicirculation. This may explain the reason for the decreasing temperature of the hot stream when the cold mass ratio is greater than 0.8. For the different vortex tubes employed in previous studies [4, 11, 14-16], different critical cold mass flow ratios were found. However, the similarities in the tendencies for change in the temperature profiles, show the reliability of these discussions and provide solid support for the proposed explanation.

\section{Geometrical effects on the tube performance}

The effects of the geometrical parameters on vortex tube performance have been investigated by many researchers, using both experimental and numerical methods. It has been reported that when different geometrical parameters were selected for testing a vortex tube, such as length and diameter of the tube, shape and size of the inlet nozzle, cold and hot exits, and structure of the tube, the temperatures of the generated cold and hot streams varied. However, there has not been an explanation that can be used to explain all the effects of the variable parameters on the tube performance. Therefore, based on the explanation proposed in this study, the geometrical effects are discussed in this paper.

\subsection{Tube length}

The effects of tube length, tube diameter and ratio of tube length over tube diameter were summarized by Yilmaz et al. [17]. It was reported that the length of 
the tube should be longer than a critical length to achieve significant temperature separation within the vortex tube. When the vortex tube is shorter than the critical value, the separating vorticities between the cold core and multi-circulation region became weaker or even disappear, and the cold flow will subsequently mix with the hot flow from the multi-circulation region. Hence, the temperature separation in a very short vortex tube will not be significant. When the length of a vortex tube approximates or is longer than the critical length, the separation of the cold region and the multi-circulation region, i.e., the hot region, is ensured by the tube length and provides a better performance of the temperature separation. The critical length is different for the vortex tube with different tube diameters.

\subsection{Tube diameter}

The performance of a vortex tube is also a function of tube diameter. When optimized, the diameter of a vortex tube can provide a perfect separation of the cold and hot regions, which therefore dictates the performance for the temperature separation. When the diameter of a vortex tube is too small or too large, mixture of cold and hot streams inside the tube or mixture of cold stream and sucked ambient air, can lead to a reduction in the temperature drop. At the hot end, weak multicirculation will be found in a vortex tube with large diameter, due to its small centrifugal force. Therefore, for a vortex tube with fixed length, there is a critical value of the tube diameter for the generation of maximum temperature separation.

\subsection{Ratio of tube length over diameter}

It has been reported that the ratio of tube length over diameter needs to be greater than 20 in order to have significant temperature separation in a vortex tube [17], and this finding agrees with the current study [3]. Once the ratio is greater than 45, it was reported that there is no further effect on the performance of the vortex tube [17]. This is likely due to the fact that the cold core region and the multicirculation region have been fully separated when the ratio of length over diameter is 
45. Therefore, it does not apparent that further lengthening of the vortex tube has any influence on the tube performance. However, with the increase of the tube length, the decrease of tangential velocity at the hot end leads to a weakening of the multicirculation region, which results in a slightly temperature drop of the hot stream.

\subsection{Tube shape}

It has been reported that a conical vortex tube can generate two streams which exhibit significant temperature differences, although the length of the conical vortex tube is much shorter than a straight vortex tube $[4,17,18]$. Optimum conical angle has been proposed by several researchers. However, there has not been an explanation for the apparent successful application of the conical tube in shortening the length of a vortex tube. According to the abovementioned explanation, the separation of the cold core region and the multi-circulation region, in a short conical vortex tube, still can be successfully achieved. Due to the conical angle of the tube, the formed, multi-circulation region in the rear part portion of the tube, will have a similar conical angle along the wall of the tube, which makes the radial dimension at the end of the multi-circulation (away from the hot end), increase. Hence, the mixture of an enlarged multi-circulation and the cold region can be avoided. Thus, a short conical vortex tube can still perform well with regard to the extent of the temperature separation.

\subsection{Vortex angle}

A new geometrical parameter, termed the vortex angle, has been investigated [19]. It has been reported that the introduced vortex angle had negative effects on the magnitude of the temperature differential achieved. Based on the proposed explanation, the introduced vortex angle leads to a decrease of the tangential velocity and an increase in the axial velocity. Since both the temperature drop and temperature rise are caused by the strong swirling flow, the decrease of the tangential 
velocity is the reason for the reduction of temperature separation in a vortex tube with a vortex angle generator installed.

\subsection{Inlet nozzle}

The strong swirling flow, which is the reason for the temperature separation in the vortex tube, is generated by the injected high speed fluid through the inlet nozzle. Therefore, the inlet nozzle, which exhibits good characteristics in generating the swirling flow, is the primary component in generating the two streams which result in large temperature difference within the vortex tube. The dimension of inlet nozzle cannot exceed a critical value, in order to generate the strong swirling flow. Generally, an increase in the inlet nozzle number leads to greater injected flow and symmetric flow in the tube, both of which lead to impressed temperature separation. Furthermore, too many inlet nozzles will cause a high back pressure inside the tube and lead to a decrease of the swirl velocity resulting in a reduction of the temperature separation. These discussions regarding the inlet nozzle agree with the results presented in [17].

\subsection{Tube exits}

The effects of the cold and hot exit on the tube performance have been discussed in a previous section: i.e., the discussion of the temperature separation at different cold mass ratio. The size of the hot end plug plays an important role in controlling the cold mass flow ratio. Furthermore, the cold exit has an obvious influence on the exhausted cold flow, including the influx of ambient air by suction. The optimum setting of the cold and hot exit is achieved by adjusting the cold mass ratio to the critical value, as discussed above. Different shapes for the hot end plug have been investigated in order to identify its influence on the tube performance $[4,20]$. It was found that the shape of the plug had negligible effects on tube performance. The primary factor in forming the multi-circulation region is the size of the plug rather 
than the shape of the plug. Hence, the effect of the plug shape was ignored in the experimental results presented here.

\subsection{Diffuser}

The diffuser has also been placed in the vortex tube to improve the tube performance [4, 21]. It was reported that the application of a diffuser after the hot exit, improved the temperature rise at the hot end and decreased the temperature drop at the cold end. The increased temperature from the hot exit is the result of further stagnation of the strong swirling flow. Measurement of hot stream temperature from a commercial vortex tube also indicates a significant improvement by installing the hot end muffler, which further stops the rotation of the flow. Application of the diffuser at the hot end also causes an increase of the exit pressure, and change the flow structure inside the vortex tube due to the increased exit pressure. There will be less flow exhausted from the hot exit, and more flow exhausted from the cold exit. In the meantime, due to the fact that more flow is forced back at the hot end, the region of multi-circulation increases and the cold core region decreases. The increase of the cold flow and the decrease of the cold core region are the reasons for a relative lowering of the temperature of the cold flow.

\section{Conclusion}

Although several explanations for the temperature separation in a vortex tube have been proposed, due to the complexity of the internal flow, there has not been a well accepted explanation, and therefore the physical processes inside the vortex tube remain unclear. This paper reports a novel explanation for the temperature separation in a vortex tube based on the descriptions of the flow structure with in the tube. The temperature drop in a vortex tube is mainly due to the pressure drop near the inlet, and the temperature rise is caused by the partial stagnation and mixture due to the structure of multi-circulation near the hot end. 
The proposed cold core region and multi-circulation region, which indicate the region of temperature drop and temperature rise, respectively, are observed and confirmed via both qualitative and quantitative investigations. The confirmation of the hypothesized flow behaviour inside a vortex tube confirms the validity of the proposed explanation. Exergy analysis in the air-operated vortex tube was performed in this study, and as such validates the stated explanation. The analysis of results from this study and that of others' show a slightly decreased peripheral exergy density in the rear part of the tube, which indicate the primary contribution of the partial stagnation and mixture due to multi-circulation, to the temperature rise. The significant drop in the peripheral exergy density in the front part of the tube, and the estimation of the temperature drop based on the forced vortex assumption, indicate the pressure gradient in the front part of the tube is the main reason for the temperature drop.

The working principle of a vortex tube is discussed according to the proposed explanation, which agrees well with the experimental results. Further discussions of the geometrical effects on the tube performance, such as cold mass flow ratio, tube length, tube diameter and inlet nozzle, have been performed to validate the explanation. The correlation between the tube performance discussed in the paper and the experimental results further underpin the validity of the explanation proposed for the temperature separation in a vortex tube.

As a conclusion, pressure gradient near the cold end is considered as the main reason for the temperature drop, whilst partial stagnation and mixture due to multicirculation is the main reason for the temperature rise. Confirmation for the explanation is presented in the paper. 


\section{Reference}

[1] Y. Xue, M. Arjomandi, R. Kelso, A critical review of temperature separation in a vortex tube, Experimental Thermal and Fluid Science, 34 (2010) 1367-1374.

[2] R. Liew, J.C.H. Zeegers, J.G.M. Kuerten, W.R. Michalek, Maxwell's Demon in the Ranque-Hilsch Vortex Tube, Physical Review Letters, 109 (2012) 054503.

[3] Y. Xue, M. Arjomandi, R. Kelso, Experimental study of the flow structure in a counter flow Ranque-Hilsch vortex tube, International Journal of Heat and Mass Transfer, 55 (2012) 5853-5860.

[4] C. Gao, Experimental study on the Ranque-Hilsch Vortex Tube, in, Technische Universiteit Eindhoven, 2005.

[5] J.P. Hartnett, E.R.G. Eckert, Experimental study of the velocity and temperature distribution in a high-velocity vortex-type flow, Heat Transfer and Fluid Mechanics Institute, Stanford University Press, (1956) 135-150.

[6] J.E. Lay, An experimental and analytical study of vortex-flow temperature separation by superposition of spiral and axial flow, part I, Trans. ASME J. Heat Transfer, 81 (1959) 202212.

[7] Y. Xue, M. Arjomandi, R. Kelso, Visualization of the flow structure in a vortex tube, Experimental Thermal and Fluid Science, 35 (2011) 1514-1521.

[8] J.G. Polihronov, A.G. Straatman, Thermodynamics of Angular Propulsion in Fluids, Physical Review Letters, 109 (2012) 054504.

[9] H.H. Bruun, Experimental Investigation of the Energy Separation in Vortex Tubes, Journal of Mechanical Engineering Science, 11 (1969) 567-582.

[10] O. Aydin, M. Baki, An experimental study on the design parameters of a counterflow vortex tube, Energy, 31 (2006) 2427-2436.

[11] P. Promvonge, S. Eiamsa-ard, Investigation on the vortex thermal separation in a vortex tube refrigerator, ScienceAsia J 2005;31(3):215-23, 31 (2005) 215-223.

[12] B. Ahlborn, S. Groves, Secondary flow in a vortex tube, Fluid Dynamics Research, 21 (1997) 73-86.

[13] U. Behera, P.J. Paul, K. Dinesh, S. Jacob, Numerical investigations on flow behaviour and energy separation in Ranque-Hilsch vortex tube, International Journal of Heat and Mass Transfer, 51 (2008) 6077-6089.

[14] M. Arjomandi, X. Yunpeng, An investigation of the effect of the hot end plugs on the efficiency of the Ranque-Hilsch vortex tube, Journal of Engineering Science and Technology (JESTEC), 2 (2007) 211-217.

[15] B.A. Shannak, Temperature separation and friction losses in vortex tube, Heat and Mass Transfer/Waerme- und Stoffuebertragung, 40 (2004) 779-785.

[16] K. Stephan, S. Lin, M. Durst, F. Huang, D. Seher, An investigation of energy separation in a vortex tube, International Journal of Heat and Mass Transfer, 26 (1983) 341-348. 
[17] M. Yilmaz, M. Kaya, S. Karagoz, S. Erdogan, A review on design criteria for vortex tubes, Heat and Mass Transfer/Waerme- und Stoffuebertragung, 45 (2009) 613-632.

[18] S. Eiamsa-ard, P. Promvonge, Review of Ranque-Hilsch effects in vortex tubes, Renewable and Sustainable Energy Reviews, 12 (2008) 1822-1842.

[19] Y. Xue, M. Arjomandi, The effect of vortex angle on the efficiency of the RanqueHilsch vortex tube, Experimental Thermal and Fluid Science, 33 (2008) 54-57.

[20] B. Markal, O. Aydın, M. Avcı, An experimental study on the effect of the valve angle of counter-flow Ranque-Hilsch vortex tubes on thermal energy separation, Experimental Thermal and Fluid Science, 34 (2010) 966-971.

[21] Y.T. Wu, Y. Ding, Y.B. Ji, C.F. Ma, M.C. Ge, Modification and experimental research on vortex tube, International Journal of Refrigeration, 30 (2007) 1042-1049. 


\section{Chapter 7}

\section{Conclusion and recommendation}

The process of generating two streams at different temperature from a vortex tube with a single injection, which is also known as the Ranque effect, energy separation, thermal separation or temperature separation, has been investigated intensively since its invention. However, the working process of a vortex tube still remains unclear, and no well accepted explanation has been proposed.

The focus of this study was to derive an acceptable explanation for the Ranque effect by evaluating previous explanations and undertaking appropriate experimental and analytical studies. The project tasks were completed by obtaining high-fidelity experimental data and analytical relationships which well supported the developed hypothesis.

\subsection{Conclusion}

The working principle of a Ranque-Hilsch vortex tube has been investigated by many researchers, and several explanations have been offered, which include pressure gradient, acoustic streaming, friction and viscosity, secondary circulation, and static temperature gradient.

As the best-known explanation, the pressure gradient of a forced vortex within a vortex tube has been stated as the reason for the thermal separation. This mechanism proposes that due to the centrifugal force generated by the strong swirling, the high pressure in the peripheral region and low pressure in the central region within a vortex tube cause the rise and drop in the temperature of the working fluid. However, as discussed in the critical review, the pressure inside a vortex tube will never be higher than the pressure at the inlet. Hence, the process of compression of the working fluid in the peripheral part of the tube is impossible, which indicates the invalidity of the above explanation. 
Similarly, the acoustic streaming was reported to be the reason for the formation of a forced vortex model within a vortex tube, and the pressure gradient of a forced vortex was suggested to be the causes of the separation of energy. However, this hypothesis cannot be accepted due to the same reason as given above, i.e., the lack of compression inside a vortex tube.

Another explanation proposed for the Ranque effect is the friction and viscosity of the working fluid, whereby the temperature of the fluid rises due to the viscous friction between the flow and the wall of the tube. However, as discussed in the critical review, the evaluation of the friction between the air flow and the wall of the tube was not in good agreement with the experimental values. Therefore this excludes the above factor as the primary underlying explanation.

Other explanations were focused on the energy transfer from the inner layer flow towards the outer layer flow. Hence, the temperature drop and the temperature rise of the exhausted fluid were induced by the loss and gain in energy of the working fluid, respectively. This energy transfer has been reported as the result of internal viscous friction between different flow layers in the process of transforming an irrotational vortex to a forced vortex. However, the lack of evidence limits the further application of this explanation. This is also the case for the roles played by the inner friction and the turbulence between different layers, where more precise measurements are required.

Another reason for the energy transfer between different flow layers was reported as the secondary circulation, which worked as a classic refrigeration system and transported the energy from the inner flow to the outer flow. However, according to the analytical result relating to the secondary circulation, which is only found within the vortex tube when the cold exit is small, an improvement of the tube performance can only be expected when the cold exit is small. Accordingly, the secondary circulation cannot be considered as the primary factor for the phenomenon of thermal separation, as it does not exist in some vortex tubes which still have the effect of temperature separation. 
The static temperature gradient within a vortex tube, which had higher temperature in the central region of the tube, was also pointed as the reason for the energy transfer outwards. However, various investigations have not reported agreement for the static temperature gradient. This, together with the small magnitude of the static temperature difference also previously reported, suggest that the static temperature gradient is not the primary factor in separating energy within a vortex tube.

Therefore, there has not been an acceptable explanation offered for the phenomenon of energy separation within a vortex tube, and this project is aimed at providing a reasonable explanation and a detailed description of the whole working process of a vortex tube.

Explanations from the literature, which relate to the effect of expansion within a vortex tube, appear relatively more consistent, and from these, it is reasonable to conclude that the expansion, i.e., pressure gradient near the injection point, is the predominant factor underlying the temperature drop. The observation that the maximum value within the temperature gradient of the peripheral flow along the tube, occurs some distance from the hot end, and leads to the novel notion that the flow structure within the rear part of the vortex tube may circulate repeatedly before escaping the tube. Based on these notions, together with agreement between the computational and experimental investigations, a novel hypothesis for the thermal separation is now proposed. Accordingly, partial stagnation and mixture due to the structure of the multi-circulation in the rear part of the tube, along with the pressure gradient near the injection point, are proposed to be the dominant factors responsible for the phenomenon of thermal separation.

The working principle of a vortex tube is described as following: When the compressed air is injected into a vortex tube tangentially, it starts rotating and moving to the other end of the tube. The inner part of the flow moves inwards and turns back in the front part of the tube. The flow in this region expands due to the lower pressure in the central part of the tube and then escapes from the cold nozzle at a temperature lower than the supplied air. In the mean time, the peripheral flow 
keeps moving to the hot end. The outer layer of the peripheral flow escapes from the gap between the control plug and the inner wall of the tube at a temperature higher than the supplied air, and the inner part of the peripheral flow is then forced back to the cold end by the plug, through the central region of the tube. Hence, on its way moving to the cold end, the central flow spreads outwards, mixes with the peripheral flow and then turns back to the hot end again. Termed multi-circulation, this specific flow structure was first hypothesised in the present research. The temperature of the peripheral flow rises due to the partial stagnation and mixing induced by the flow structure of the multi-circulation. Illustrated flow behaviour within a vortex tube is presented in chapter 6 (Figure 2, 6.3. The working principle of a vortex tube).

To validate the proposed hypothesis, a clear understanding of the flow structure inside a vortex tube is required, which confirms the proposed flow structure. This applies especially to both the region near the injection point in which sudden expansion is considered as the governing process, and the region near the exit in which multi-circulation may be formed. One issue, which may impact on the validity of the explanation, is the energy transfer between different layers. If the energy transfer between layers is significant, it indicates that the energy separation is caused by the energy transfer from the inner fluid towards the peripheral fluid. On the contrary, if the energy transferred from the inner flow to the outer flow is negligible, the validity of the proposed hypothesis will be strengthened. Therefore, further validation of the proposed hypothesis would come from more precise measurements of the extent of energy transfer between different layers. The explanation offered in this research can be also supported by its application in estimating the temperature drop, explaining the working process of a vortex tube and predicting the effects of various geometric parameters.

The proposed hypothesis was firstly validated by confirming the flow structure. Flow visualization methods were employed in this study in order to obtain a detailed understanding of the flow patterns within the vortex tube. To achieve this, a transparent vortex tube with a single injection port was designed, which was suitable for flow visualization experiments using water as the working medium. The use of water as the working fluid in the vortex tube enabled the successful visualization of 
the internal flow field by reducing the injecting velocity and maintaining the same Reynolds number at the inlet. Moreover, the steady pressure gradient in the rear part of the tube ensured the reliability of the visualization results using water inside the vortex tube. Several visualizing materials, including dye, air bubble, hydrogen bubble, and tracer particles, were injected into the vortex tube, enabling the internal flow field to be visualized.

The assumed flow structure, i.e., the turn back of the flow in the cold core, and the multi-circulation in the hot region, were observed using the above flow visualization techniques. Hence, the explanation offered here was firstly supported by the confirmation of the proposed flow structure within a water-operated vortex tube. Furthermore, according to the swirl velocity reading based on the visualization results, an irrotational vortex was observed near the hot end, which caused the outwards movement of the multi-circulation. The volume flow rate along the vortex tube calculated from the axial velocity distribution, also indicated the existence and position of the multi-circulation region. These analytical results further strengthen the validity of the proposed explanation.

The flow behaviour within the vortex tube was further confirmed by the precise measurement results of the flow properties inside an air-operated vortex tube. Because the phenomenon of thermal separation occurs in a vortex tube with compressible working fluid, instead of an incompressible fluid, compressed air was employed as the working medium in further experiments. In order to have good comparison between the visualization results, the air-operated vortex tube was designed with a similar geometry, including the cold exit, hot exit and injection port.

An understanding of the flow mechanisms inside the optimised air-operated vortex tube was obtained from the detailed measurements of the flow properties, which included 3-D velocity distributions, pressure profiles and temperature profiles. Precise analysis of the 3-D velocity profiles revealed evidence in support of the proposed flow behaviour, including the inwards movement of the flow near the injection port, the turn back in the cold core region, and the outwards movement of the multi-circulation. The turbulence intensity, static pressure, density and static 
temperature gradients along the tube were analysed with regard to the hypothesised flow structure, and were in good agreement. Therefore, the proposed flow structure has been fully supported by the experimental results in this study and further strengthens the overall explanation for the Ranque effect. Additionally, the irrotational vortex near the hot end that was observed from the flow visualization was confirmed by the transition of a forced vortex at the cold end, to an irrotational vortex at the hot end in the air-operated vortex tube. This transition was supported by the static pressure gradient along the tube. The agreement between the swirl velocities in both water-operated and air-operated vortex tubes further supported the postulated flow structure.

In the flow visualization study, the oscillation of the rotating axis was observed, which was caused by the single the injection ports. The oscillation of the rotating axis was also indicated by the radial velocity distributions in the air-operated vortex tube. Agreeing with numerical investigations reported by other researchers, the oscillation of the rotating axis does not have significant impact on the tube performance. Instead, it was found that the oscillation induced difficulties in the flow property measurements, which can be minimised in further study.

Analysis was also performed on the energy transfer between different layers of flow by defining the exergy density gradient along the tube. Calculated from the results of experimental measurements of the flow properties, the exergy component contains three parts, i.e., the kinetic energy, internal energy and increased entropy, and together these represent the maximum available energy level within the vortex tube. Then the energy transfer between layers can be identified using the calculated exergy profiles. It has been noted that the exergy density in the peripheral region decreases dramatically from the inlet. This is due to the filling of the central part of the vortex tube by the peripheral flow and demonstrates the existence of the expansion. In the rear part of the vortex tube, the slightly reduced exergy density of the peripheral flow along the tube indicates that there is negligible energy transferred outwards on its way towards the hot end. Hence, the temperature rise near the hot end is mainly caused by the mixture and partial stagnation of the axial flow via the structure of the multi-circulation. This is in contrast to those hypotheses that the 
Ranque effect is caused by the energy transfer from the inner layer flow towards the outer layer flow. The analysis of the detailed exergy density undertaken in this study also agrees with the proposed flow structure and provides further support for the explanation offered in this research.

To clarify the independence of the proposed explanation from the geometry and setup of this experimental investigation, the exergy analysis has also been carried out using the flow properties reported in other experimental investigations. A nondimensionalized value of the exergy density, termed the exergy density ratio, was calculated using the relationship between the actual value of exergy density and exergy density at the inlet, which is also the maximum value in the tube. In this way, the exergy density within the vortex tubes used in different experimental investigations can be analysed. A good agreement of the exergy profiles in these experimental investigations with these of previously reported studies has been observed, and therefore further supports the proposed explanation. The total exergy profiles at the axial cross section along the tube have also been calculated in this study. The consistency between the exergy density ratio and the total exergy profiles further supports the explanation.

The explanation was further validated by, firstly the estimation of the temperature drop from a vortex tube, secondly the successful application of the proposed explanation in explaining the whole working process of a vortex tube, and thirdly agreement between the theoretical analysis and experimental observations of the geometrical effects. An estimation of the temperature drop from a vortex tube was performed using the forced vortex model near the cold end. The good agreement between the predicted and experimental values further supports the validity of the proposed explanation. The working principle of a vortex tube was fully discussed based on the proposed explanation, and this was strongly correlated with the experimental performance of an actual vortex tube. Studies aimed at optimising the tube performance were also undertaken and these revealed the significant influence the geometrical parameters had on the tube performance. These geometrical effects, together with those collected from other experimental investigations, were then compared with the analytical results of the geometrical effects based on the proposed 
explanation. The significant correlation, which was obtained between the experimental and theoretical analysis with regard to the influence of the geometric parameters, gave further support to the proposed hypothesis.

Therefore, the novel explanation for the thermal separation phenomenon observed in a vortex tube, which was forwarded in this study, was well supported by the confirmation of the flow behaviour, the theoretical analysis of the exergy profiles, the accuracy of the predicted temperature drop, and the successful application of the hypothesis presented in explaining the geometrical effects observed in the vortex tube system.

\subsection{Recommendation}

To gain more accurate observations of the flow structure within the vortex tube and fully understand the detailed behaviour flow within the tube, different measurement methodologies are recommended. In all previous experimental investigations, the flow properties inside a vortex tube were measured by inserting a particular measuring device into the vortex tube. Hence, perturbation to the internal flow field would have been induced by the inserted measuring device, which led to unavoidable measurement errors of the actual internal flow. Therefore, the pure flow field within a vortex tube could be obtained by applying non-invasive measurement technologies without any insertion or disturbulence to the flow field within the vortex tube.

In this regard:

- PIV (Particle Image Velocimetry) is recommended for the velocity measurement in a transparent vortex tube due to its high accuracy and lack of the need to insert any measuring device into the vortex tube. Indeed, PIV was firstly selected to measure the internal velocity profiles within a vortex tube. However, due to the limitations in this experimental investigation, which includes the strong swirling flow, the geometrical limitations of the vortex tube, optical distortion of the curved wall of the tube, the requirement of three-dimensional velocity profiles along the tube, and the centrifugal 
acceleration of the tracing particles, velocity profiles were measured using alternative devices.

- A thermal camera is also suggested for temperature measurement due to its high accuracy and its ability to undertake measurement from outside of the vortex tube. This may be achieved by adding some temperature-sensitive particles into the internal flow field.

- Any potential methodology that consists of not inserting any device into the tube, for the pressure measurement within the vortex tube, is suggested as a further recommendation.

Furthermore, as the influence of the oscillation on the measurements, which is reported in this research, a proper number of injection ports is recommended to provide optimised tube performance and minimised measurement uncertainty at the same time. 